\title{
Cascade Cyclizations and Couplings Involving Nickel Enolates
}

Gireesh M. Mahandru, Andy R. L. Skauge, Sanjoy K. Chowdhury, Kande K. D. Amarasinghe, Mary Jane Heeg, and John Montgomery*

Department of Chemistry, Wayne Sate University, Detroit, MI 48202-3489

\section{Supporting Information}

General Information. All reagents were used as received unless otherwise noted. Tetrahydrofuran (THF) was either freshly distilled from sodium/benzophenone ketyl or treated under nitrogen through a solvent purification system (Innovative Technology, Inc., Model \# SPS-400-3). TMEDA (tetramethylethylenediamine) was distilled from calcium hydride. Benzaldehyde and methyl iodide were freshly distilled. $\mathrm{Ni}(\mathrm{COD})_{2}$ (Strem Chemicals, Inc.; used as received) was stored and weighed in an inert atmosphere glovebox. All reactions were conducted in flame-dried glassware under an oxygen-free atmosphere of argon or nitrogen. Analytical thin layer chromatography (TLC) were performed using EM Science $0.25 \mathrm{~mm}$ Silica gel $60-\mathrm{F}$ plates. Visualization of the developed chromatogram was accomplished with UV light and aqueous potassium permanganate, acidic $p$-anisaldehyde, or ethanolic vanillin stains. Column chromatography was done with silica gel 60 (EM Science) 0.040-0.063 mm (230-400 mesh ASTM), unless otherwise noted. ${ }^{1} \mathrm{H}$ and ${ }^{13} \mathrm{C}$ NMR spectra were obtained in deuterochloroform $\left(\mathrm{CDCl}_{3}\right)$, unless otherwise noted, on a Varian Unity 300, Varian Mercury 400, or Varian Unity $500 \mathrm{MHz}$ instrument. Chemical shifts of ${ }^{1} \mathrm{H}$ NMR spectra were recorded in parts per million ( $\mathrm{ppm})$ on the $\delta$ scale from an internal standard of residual chloroform $(7.27 \mathrm{ppm})$. NMR data are reported as follows: chemical shift, multiplicity $(\mathrm{s}=$ singlet, $\mathrm{d}=$ doublet, $\mathrm{t}=$ triplet, $\mathrm{q}=$ quartet, quint $=$ quintet, $\mathrm{sext}=$ sextet, sept $=$ septet, $\mathrm{m}=$ multiplet, and $\mathrm{br}=$ broad $)$, coupling constant in Hertz $(\mathrm{Hz})$, and integration. Chemical shifts of ${ }^{13} \mathrm{C}$ NMR spectra are reported in ppm from the central peak of $\mathrm{CDCl}_{3}(77.0 \mathrm{ppm})$ on the $\delta$ scale. Infrared (IR) spectra were recorded on a 
Nicolet Avatar 320 FT-IR spectrometer. High Resolution mass spectra (HMRS) were obtained on a Kratos MS 80 mass spectrometer by the Central Instrumentation Facility, Department of Chemistry, Wayne State University, Detroit, Michigan. $\mathrm{rt}=$ room temperature, $\mathrm{mp}=$ melting point, $\mathrm{h}$ or $\mathrm{hr}=$ hour, $\min =$ minutes, $\mathrm{eq}=$ equivalents, EtOAc $=$ ethyl acetate, $\mathrm{Hex}=$ hexanes, $\mathrm{COD}=1,5$-cyclooctadiene.

Compounds (in order as they appear in Table 1) 9a, 9b, 9a', 11a, 9c, 10a, 10b, 11b, and Table 2 (entries $\mathbf{1}$ and $\mathbf{4}$ ) were formed as previously reported. ${ }^{1}$

\section{General Procedure for Table 1 and Table 2:}

\section{General Procedure for the $\mathrm{Ni}(\mathrm{COD})_{2} /$ tmeda Promoted [3+2] Cyclization of Enals} and Enones:

To a solution of $\mathrm{Ni}(\mathrm{COD})_{2}$ (1 equiv) in $\mathrm{THF}(0.05 \mathrm{M})$ was added dropwise tetramethylethylene diamine (tmeda) (1 equiv) at room temperature. After stirring for 510 min at $\mathrm{rt}$, the reaction mixture became bright yellow and was cooled to $0^{\circ} \mathrm{C}$. A solution of enal or enone at $0^{\circ} \mathrm{C}$ (RT or warmer especially for the silane derivatives) in THF $(0.05 \mathrm{M})$ was added dropwise, and the reaction mixture was stirred at $0^{\circ} \mathrm{C}$ and allowed to warm to room temperature or until TLC analysis indicated disappearance of the enal or enone.

\section{Aqueous Work-up} enals (entries 1-4, and 6, Table 1): The reaction mixture was quenched with aqueous $\mathrm{NH}_{4} \mathrm{Cl} / \mathrm{NH}_{4} \mathrm{OH}\left(\mathrm{pH}\right.$ 8) buffer and extracted twice with $\mathrm{Et}_{2} \mathrm{O}$. The combined organic layers were washed with brine, dried over $\mathrm{MgSO}_{4}$, filtered, and concentrated, and the

${ }^{1}$ Chowdhury, S. K.; Amarasinghe, K.K.D.; Heeg, M. J.; Montgomery, J. J. Am. Chem. Soc., 2000, 122, 6775 . 
residue was purified by column chromatography on silica gel.

Enones (entries 8-10, Table 1): The reaction mixture was poured over a slurry of silica gel in $\mathrm{CH}_{2} \mathrm{Cl}_{2}$ in air and was stirred for $30 \mathrm{~min}$, filtered, and concentrated. The residue was dissolved in $\mathrm{THF} / \mathrm{H}_{2} \mathrm{O}$ (9:1), $p$-toluenesulfonic acid (1 equiv) was added, and the mixture was stirred for $10 \mathrm{~min}$ at $\mathrm{rt}$. The reaction mixture was quenched with saturated $\mathrm{NaHCO}_{3}$ and was extracted twice with $\mathrm{Et}_{2} \mathrm{O}$. The combined organic layers were washed with brine, dried over $\mathrm{MgSO}_{4}$, filtered, and concentrated, and the residue was purified by column chromatography on silica gel.

Methanol Work-up for enones (entries 5,7 and 11, Table 1): The reaction mixture was quenched with excess anhydrous methanol and stirred for 30 minutes. The reaction mixture was poured in air over a pad of silica gel wetted with ether on a cintered glass funnel and filtered with suction. The solvent was removed by rotary evaporation and the filtrate subjected to column chromatography on silica gel.

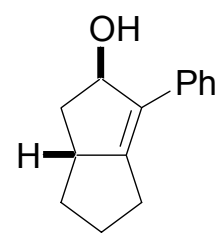

9a

\section{Entry 1: $\left(3 R^{*}, 5 R^{*}\right)-2-(P h e n y l) b i c y c l o[3.3 .0] o c t-1-e n-3-o l ~(9 a)$}

Following the general procedure, 8-(phenyl)oct-2-en-7-ynal (135 mg, $0.681 \mathrm{mmol}$ ), $\mathrm{Ni}(\mathrm{COD})_{2}(187 \mathrm{mg}, 0.681 \mathrm{mmol})$, and tmeda $(102 \mu \mathrm{L}, 0.681 \mathrm{mmol})$ were stirred for $2 \mathrm{~h}$ at $0^{\circ} \mathrm{C}$. Product 9a $(112 \mathrm{mg}, 82 \%)$ was obtained as colorless oil after $\mathrm{SiO}_{2}$ chromatography (EtOAc/Hex: 1/4). ${ }^{1} \mathrm{H}$ NMR $\left(400 \mathrm{MHz}, \mathrm{CDCl}_{3}\right) \delta 7.48(\mathrm{~d}, J=8.0 \mathrm{~Hz}$, 2H), $7.36(\mathrm{t}, J=7.6 \mathrm{~Hz}, 2 \mathrm{H}), 7.20-7.26(\mathrm{~m}, 1 \mathrm{H}), 5.47$ (d, $J=6.4 \mathrm{~Hz}, 1 \mathrm{H}), 3.33-3.38$ (m, 1H), 2.55-2.62 (m, 1H), 2.37-2.46 (m, 1H), 1.97-2.17 (m, 5H), 1.79 (quint, $J=6.9 \mathrm{~Hz}$, 1H), 0.95-1.06 (m, 1H); ${ }^{13} \mathrm{C}$ NMR (100 MHz, $\left.\mathrm{CDCl}_{3}\right) \delta 156.5,135.4,131.8,128.8$, 127.1, 126.7, 83.3, 52.2, 40.1, 32.0, 29.6, 26.0; IR (film, $\mathrm{cm}^{-1}$ ) 3394; HRMS (EI) $\mathrm{m} / \mathrm{z}$ 
calcd for $\mathrm{C}_{14} \mathrm{H}_{16} \mathrm{O}\left[\mathrm{M}^{+}\right] 200.1197$, found 200.1199. Irradiation of the methine signal at $\delta$ $5.47(\mathrm{C} \underline{\mathrm{H}}-\mathrm{OH})$ resulted in a $4.6 \%$ NOE enhancement to the methylene signal at $\delta 1.79$ ppm. Irradiation of the other methylene signal at $\delta 1.97-2.17$ resulted in a $2.7 \%$ NOE enhancement of the methine signal at $\delta 3.33-3.38 \mathrm{ppm}$ (bridgehead- $\mathrm{CH}$ ), and 5.9\% NOE enhancement to the methylene signal at $\delta 1.79 \mathrm{ppm}$.

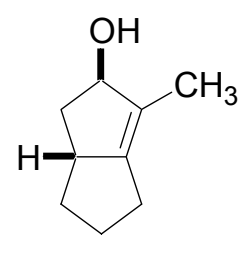

9b

\section{Entry $2:\left(3 R^{*}, 5 R^{*}\right)-2-($ Methyl)bicyclo[3.3.0]oct-1-en-3-ol (9b)}

Following the general procedure, non-2-en-7-ynal (62 mg, $0.45 \mathrm{mmol}), \mathrm{Ni}(\mathrm{COD})_{2}(125$ $\mathrm{mg}, 0.45 \mathrm{mmol})$, and tmeda $(68 \mu \mathrm{L}, 0.45 \mathrm{mmol})$ were stirred $2 \mathrm{~h}$ at $0^{\circ} \mathrm{C}$. Product $9 \mathrm{~b}(45$ mg, 72\%) was obtained as colorless oil after $\mathrm{SiO}_{2}$ chromatography $\left(\mathrm{Et}_{2} \mathrm{O} / \mathrm{Hex}: 1 / 4\right) .{ }^{1} \mathrm{H}$ NMR $\left(400 \mathrm{MHz}, \mathrm{CDCl}_{3}\right) \delta 4.65(\mathrm{~d}, J=6.8 \mathrm{~Hz}, 1 \mathrm{H}), 3.02-3.05(\mathrm{~m}, 1 \mathrm{H}), 2.05-2.13(\mathrm{~m}$, 2H), 1.95-2.03 (m, 2H), 1.85-1.94 (m, 2H), 1.69 (s, 3H), 1.63-1.67 (m, 1H), 1.48-1.53 (br s, $1 \mathrm{H}), 0.82-0.94(\mathrm{~m}, 1 \mathrm{H}) ;{ }^{13} \mathrm{C} \mathrm{NMR}\left(100 \mathrm{MHz}, \mathrm{CDCl}_{3}\right) \delta$ 152.8, 128.6, 87.1, 49.7, 40.8, 32.6, 28.6, 22.4, 12.6; IR (film, $\mathrm{cm}^{-1}$ ) 3429; HRMS (EI) $\mathrm{m} / z$ calcd for $\mathrm{C}_{9} \mathrm{H}_{14} \mathrm{O}\left[\mathrm{M}^{+}\right]$ 138.1044 found 138.1044 . The stereochemistry was assigned by analogy to 9a.

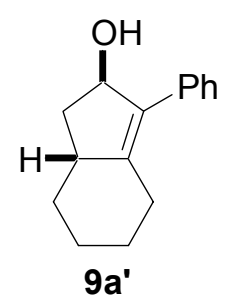

\section{Entry 3 : (1R*,8R*)-7-(Phenyl)bicyclo[4.3.0]non-6-en-8-ol (9a')}

Following the general procedure, 9-(phenyl)non-2-en-8-ynal $(82 \mathrm{mg}, 0.39 \mathrm{mmol})$ in THF (0.1 M, $3.8 \mathrm{~mL}), \mathrm{Ni}(\mathrm{COD})_{2}(106 \mathrm{mg}, 0.386 \mathrm{mmol})$, and tmeda $(58 \mu \mathrm{L}, 0.386 \mathrm{mmol})$ were stirred $18 \mathrm{~h}$ at $0^{\circ} \mathrm{C}$. The reaction mixture was filtered through $\mathrm{SiO}_{2}$ and concentrated. 
Product 9a' (33 mg, 40\%) and product 11a (15 mg, 18\%) were obtained as colorless oils after $\mathrm{SiO}_{2}$ chromatography (EtOAc/Hex, 1:9). For compound 9a': ${ }^{1} \mathrm{H}$ NMR (500 MHz, $\left.\mathrm{C}_{6} \mathrm{D}_{6}\right) \delta 7.32-7.34(\mathrm{~m}, 2 \mathrm{H}), 7.17-7.20(\mathrm{~m}, 2 \mathrm{H}), 7.06-7.09(\mathrm{~m}, 1 \mathrm{H}), 4.91(\mathrm{~d}, J=8.0 \mathrm{~Hz}$, 1H), $2.67(\mathrm{td}, J=14,2 \mathrm{~Hz}, 1 \mathrm{H}), 2.54(\mathrm{sext}, J=6.0 \mathrm{~Hz}, 1 \mathrm{H}), 1.99$ (ddd, $J=14.0,7.5,1.5$ $\mathrm{Hz}, 1 \mathrm{H}), 1.80-1.84(\mathrm{~m}, 1 \mathrm{H}), 1.73(\mathrm{dt}, J=14.0,5.0 \mathrm{~Hz}, 1 \mathrm{H}), 1.62$ (quint, $J=7.0 \mathrm{~Hz}, 1 \mathrm{H}$ ), 1.47-1.53 (m, 2H), 1.28, (br s, 1H), 1.11 (tq, $J=3.5,13.5 \mathrm{~Hz}, 1 \mathrm{H}), 0.94$ (tq, $J=13.5,4.5$ $\mathrm{Hz}, 1 \mathrm{H}), 0.78(\mathrm{dq}, J=12.5,3.0 \mathrm{~Hz}, 1 \mathrm{H}) ;{ }^{13} \mathrm{C} \mathrm{NMR}\left(125 \mathrm{MHz}, \mathrm{CDCl}_{3}\right) \delta 146.3,136.2$, 135.3, 128.8, 128.6, 127.0, 79.2, 45.3, 40.4, 36.0, 27.6, 26.9, 25.9; IR (film, cm-1) 3387; HRMS (EI) $m / z$ calcd for $\mathrm{C}_{15} \mathrm{H}_{18} \mathrm{O}\left[\mathrm{M}^{+}\right] 214.1353$ found 214.1353. Irradiation of the methine signal at $\delta 4.91(\mathrm{CH}-\mathrm{OH})$ resulted in a $2.9 \%$ NOE enhancement to the methylene signal at $\delta 1.62 \mathrm{ppm}$. Irradiation of the other methylene signal at $\delta 1.99$ resulted in a $2.7 \%$ NOE enhancement of the methine signal at $\delta 2.54 \mathrm{ppm}$ (bridgehead-CH), and 12.8\% NOE enhancement to the methylene signal at $\delta 1.62 \mathrm{ppm}$.

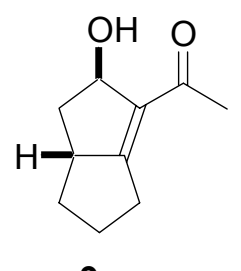

9c

\section{Entry 4 : (3R*,5R*)-2-(Acetyl)bicyclo[3.3.0]oct-1-en-3-ol (9c)}

Following the general procedure, 9-(oxo)dec-2-en-7-ynal (67 mg, $0.41 \mathrm{mmol}), \mathrm{Ni}(\mathrm{COD})_{2}$ (112 mg, $0.408 \mathrm{mmol})$, and tmeda $(62 \mu \mathrm{L}, 0.41 \mathrm{mmol})$ were stirred $2 \mathrm{~h}$ at $0^{\circ} \mathrm{C}$. Product $9 \mathrm{c}$ $(10 \mathrm{mg}, 15 \%)$ was obtained as crystalline solid (mp. 59-60 $\left.{ }^{\circ} \mathrm{C}\right)$ after $\mathrm{SiO}_{2}$ chromatography (EtOAc/Hex: 1/1). ${ }^{1} \mathrm{H}$ NMR (500 MHz, $\left.\mathrm{CDCl}_{3}\right) \delta 5.28(\mathrm{~d}, J=6.5 \mathrm{~Hz}, 1 \mathrm{H}), 3.40-3.46$ (m, 1H), 2.50-2.65 (m, 2H), 2.30 (s, 3H), 2.26 (br s, 1H), 2.15-2.22 (m, 1H), 2.01-2.09 (m, $3 \mathrm{H}), 1.65-1.71(\mathrm{~m}, 1 \mathrm{H}), 1.06-1.14(\mathrm{~m}, 1 \mathrm{H}) ;{ }^{13} \mathrm{C} \mathrm{NMR}\left(125 \mathrm{MHz}, \mathrm{CDCl}_{3}\right) \delta$ 197.1, 174.5, 134.7, 81.8, 54.2, 37.8, 31.3, 29.3, 27.0; IR (film, $\mathrm{cm}^{-1}$ ) 3429, 1640; HRMS (EI) $\mathrm{m} / \mathrm{z}$ 
calcd for $\mathrm{C}_{10} \mathrm{H}_{14} \mathrm{O}_{2}\left[\mathrm{M}^{+}\right] 166.099$ found 166.0991 . The stereochemistry was assigned by analogy to $9 \mathbf{a}$.

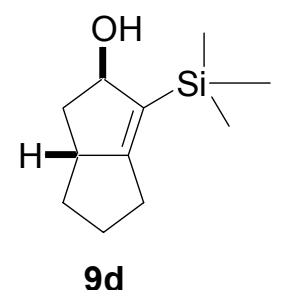

Entry 5: (3R*,5R*)-2-(Trimethylsilanyl)bicyclo[3.3.0]oct-1-en-3-ol (9d)

Following the general procedure, 8-trimethylsilanyl-oct-2-en-7-ynal (38 mg, $0.19 \mathrm{mmol})$, $\mathrm{Ni}(\mathrm{COD})_{2}(54 \mathrm{mg}, 0.19 \mathrm{mmol})$ and tmeda $(30 \mu \mathrm{L}, 0.19 \mathrm{mmol})$ were stirred for $2 \mathrm{~h}$ at $\mathrm{rt}$. The reaction mixture was subsequently heated to $45^{\circ} \mathrm{C}$ for 4 hours and then at $55^{\circ} \mathrm{C}$ for another 4 hours. The reaction mixture was cooled to $\mathrm{rt}$ before anhydrous methanol (1 $\mathrm{mL}$ ) was added and stirred for $30 \mathrm{~min}$. After chromatography (EtOAc/Hex, 1:10), product $9 \mathrm{~d}(21 \mathrm{mg}, 0.11 \mathrm{mmol}, 55 \%)$ was obtained as a light yellow oil. A small portion ( $5 \mathrm{mg}, 13 \%)$ of starting material was also recovered. ${ }^{1} \mathrm{H} \mathrm{NMR}\left(400 \mathrm{MHz}, \mathrm{CDCl}_{3}\right) \delta 5.00$ $(\mathrm{d}, J=6.0 \mathrm{~Hz}, 1 \mathrm{H}), 3.12-3.20(\mathrm{~m}, 1 \mathrm{H}), 2.27-2.34(\mathrm{~m}, 1 \mathrm{H}), 2.11-2.20(\mathrm{~m}, 1 \mathrm{H}), 1.87-2.10$ (m, 4H), $1.52(\mathrm{ddd}, J=13.4,7.2,5.6 \mathrm{~Hz}, 1 \mathrm{H}), 1.27$ (br s, $1 \mathrm{H}), 0.86-0.96(\mathrm{~m}, 1 \mathrm{H}), 0.13$ (s, $9 \mathrm{H}) ;{ }^{13} \mathrm{C}$ NMR $\left(100 \mathrm{MHz}, \mathrm{CDCl}_{3}\right) \delta 127.5,131.9,87.3,52.7,41.8,31.7,29.3,25.0,-0.5$; IR (film, $\mathrm{cm}^{-1}$ ) 3365, 1716, 1621, 1446, 1247, 838; HRMS (EI) $\mathrm{m} / z$ calcd for $\mathrm{C}_{11} \mathrm{H}_{20} \mathrm{OSi}$ $\left[\mathrm{M}^{+}\right]$196.1283, found 196.1284. 


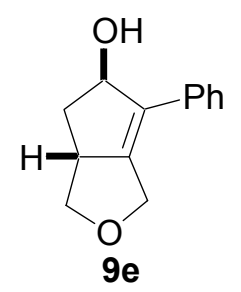

\section{Entry 6 : (3R*,5R*)-2-(Phenyl)-7-oxo-bicyclo[3.3.0]oct-1-en-3-ol (9e)}

Following the general procedure, 4-(3-Phenyl-prop-2-ynyloxy)-but-2-enal (72 mg, 0.36 mmol), $\mathrm{Ni}(\mathrm{COD})_{2}(98 \mathrm{mg}, 0.36 \mathrm{mmol})$ and tmeda $(54 \mu \mathrm{L}, 0.36 \mathrm{mmol})$ were stirred for $2 \mathrm{~h}$ at $0^{\circ} \mathrm{C}$. Product $9 \mathrm{e}(57 \mathrm{mg}, 0.28 \mathrm{mmol}, 78 \%)$ was obtained as a white crystalline solid (mp 76-77 ${ }^{\circ} \mathrm{C}$ ) after chromatography (EtOAc/Hex, 1:5). ${ }^{1} \mathrm{H}$ NMR $\left(400 \mathrm{MHz}, \mathrm{CDCl}_{3}\right)$ $\delta$ 7.36-7.40 (m, 2H), 7.26-7.29 (m, 3H), $5.61(\mathrm{~d}, J=6.0 \mathrm{~Hz}, 1 \mathrm{H}), 4.60(\mathrm{~d}, J=14.0 \mathrm{~Hz}$, $1 \mathrm{H}), 4.42(\mathrm{~d}, J=14.4 \mathrm{~Hz}, 1 \mathrm{H}), 4.15-4.18(\mathrm{~m}, 1 \mathrm{H}), 3.76-3.82(\mathrm{~m}, 1 \mathrm{H}), 3.11(\mathrm{dd}, J=$ 10.6, 7.4 Hz, 1H), 2.14 (dd, $J=13.6,6.4 \mathrm{~Hz}, 1 \mathrm{H}), 2.07$ (br m, 1H), 1.88-1.95 (m, 1H); ${ }^{13} \mathrm{C}$ NMR $\left(100 \mathrm{MHz}, \mathrm{CDCl}_{3}\right) \delta 150.8,134.0,133.8,129.1,127.8,127.3,83.8,72.2,65.4$, 51.3, 37.1 IR (film, $\mathrm{cm}^{-1}$ ) 3366, 1496, 1442, 1183, 1091, 1012; HRMS (EI) $\mathrm{m} / \mathrm{z}$ calcd for $\mathrm{C}_{13} \mathrm{H}_{14} \mathrm{O}_{2}\left[\mathrm{M}^{+}\right]$202.0994, found 202.0993.

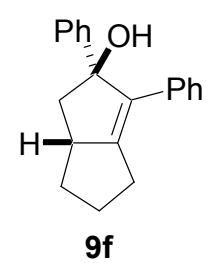

\section{Entry $7:\left(3 R^{*}, 5 R^{*}\right)-2,3-(D i p h e n y l) b i c y c l o[3.3 .0]$ oct-1-en-3-ol (9f)}

Following the general procedure, $\mathrm{Ni}(\mathrm{COD})_{2}(102 \mathrm{mg}, 0.371 \mathrm{mmol}$, 1eq.), $6 \mathrm{~mL} \mathrm{THF}$, tmeda (60 $\mu \mathrm{L}, 0.40 \mathrm{mmol}$, 1eq.), and 1,8-Diphenyl-oct-2-en-7-yn-1-one (102 mg, 0.371 mmol) as a solution in $6 \mathrm{~mL}$ THF were stirred at $0^{\circ} \mathrm{C} \rightarrow \mathrm{rt}$. After 3 hours, the reaction was 
quenched with $10 \mathrm{~mL}$ anhydrous methanol. The reaction mixture was filtered through a pad of Celite and dried over anhydrous magnesium sulfate. Column chromatography (10:1 Hex/EtOAc) afforded the colorless oil, 9 f (43.6 mg, $0.158 \mathrm{mmol}, 52 \%) .{ }^{1} \mathrm{H}$ NMR (400 MHz, $\left.\mathrm{C}_{6} \mathrm{D}_{6}\right): \delta 7.55-7.58(\mathrm{~m}, 2 \mathrm{H}), 7.32(\mathrm{~d}, J=7.2 \mathrm{~Hz}, 2 \mathrm{H}), 7.08-7.11(\mathrm{~m}, 2 \mathrm{H}), 6.94-$ $7.00(\mathrm{~m}, 3 \mathrm{H}), 6.85(\mathrm{t}, J=7.2 \mathrm{~Hz}, 1 \mathrm{H}), 2.78-2.86(\mathrm{~m}, 1 \mathrm{H}), 2.29-2.40(\mathrm{~m}, 2 \mathrm{H}), 2.12-2.18$ $(\mathrm{m}, 1 \mathrm{H}), 1.96(\mathrm{~s}, 1 \mathrm{H}), 1.82-1.90(\mathrm{~m}, 2 \mathrm{H}), 1.61-1.73(\mathrm{~m}, 2 \mathrm{H}), 0.85-0.96(\mathrm{~m}, 1 \mathrm{H}) ;{ }^{13} \mathrm{C}$ NMR (100 MHz, $\left.\mathrm{C}_{6} \mathrm{D}_{6}\right): \delta 156.7,147.8,135.9,133.6,128.1,128.05,128.02,126.3$, 126.1, 125.9, 94.8, 51.6, 50.4, 31.8, 29.1, 25.7; IR (film, $\left.\mathrm{cm}^{-1}\right)$ : 3454(br w), 1671(m), 1600(m), 1495(m), 1446(m), 1061(m), 975(m), 909(s), 760(s), 732(s), 699(s); HRMS (EI) $m / z$ calcd for $\mathrm{C}_{20} \mathrm{H}_{20} \mathrm{O}\left[\mathrm{M}^{+}\right] 276.1514$, found 276.1514 .

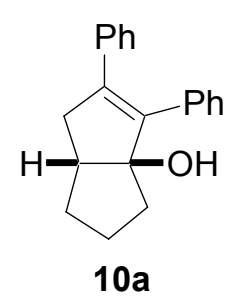

\section{Entry 8: (1R*,5S*)-2,3-(Diphenyl)bicyclo[3.3.0]oct-2-en-1-ol (10a)}

Following the general procedure, 1,8-(diphenyl)oct-2-en-7-yn-1-one $(68 \mathrm{mg}, 0.248$ $\mathrm{mmol}), \mathrm{Ni}(\mathrm{COD})_{2}(68 \mathrm{mg}, 0.248 \mathrm{mmol})$, and tmeda $(37 \mu \mathrm{L}, 0.248 \mathrm{mmol})$ were stirred for $3 \mathrm{~h}$ at $0^{\circ} \mathrm{C}$. Product 10a $(35 \mathrm{mg}, 53 \%)$ was obtained as crystalline solid $\left(\mathrm{mp} 113-114^{\circ} \mathrm{C}\right)$ after chromatography (EtOAc/Hex, 1/4). ${ }^{1} \mathrm{H}$ NMR $\left(500 \mathrm{MHz}, \mathrm{CDCl}_{3}\right) \delta$ 7.25-7.29 (m, 5H), 7.12-7.17 (m, 5H), 3.19 (dd, $J=17.0,8.5 \mathrm{~Hz}, 1 \mathrm{H}), 2.57-2.62(\mathrm{~m}, 1 \mathrm{H}), 2.53$ (dd, $J=$ $16.8,3.3 \mathrm{~Hz}, 1 \mathrm{H}), 2.19-2.26(\mathrm{~m}, 1 \mathrm{H}), 1.87-1.92(\mathrm{~m}, 1 \mathrm{H}), 1.76-1.83(\mathrm{~m}, 1 \mathrm{H}), 1.61-1.74$ (m, 2H), 1.51 (sext, $J=6.2 \mathrm{~Hz}, 1 \mathrm{H}) ;{ }^{13} \mathrm{C} \mathrm{NMR}\left(125 \mathrm{MHz}, \mathrm{CDCl}_{3}\right) \delta 141.1,139.7,137.2$, 129.8, 128.6, 128.5, 128.1, 127.4, 127.2, 98.7, 49.0, 42.1, 39.6, 35.2, 26.0; IR (film, cm1) 3339; HRMS (EI) $\mathrm{m} / z$ calcd for $\mathrm{C}_{20} \mathrm{H}_{20} \mathrm{O}\left[\mathrm{M}^{+}\right] 276.1514$ found 276.1517. The structure of 10a was proven by single-crystal X-ray analysis. The author has deposited 
atomic coordinates for $\mathbf{1 0 a}$ with the Cambridge Crystallographic Data Centre. The coordinates can be obtained, on request, from the Director, Cambridge Crystallographic Data Centre, 12 Union Road, Cambridge, CB2 1EZ, UK.

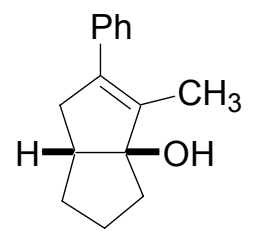

$10 \mathrm{~b}$

Entry 9: (1R*,5S*)-2-Methyl-3-(phenyl)bicyclo[3.3.0]oct-2-en-1-ol (10b)

Following the general procedure, 1-(phenyl)non-2-en-7-yn-1-one (105 mg, $0.495 \mathrm{mmol})$, $\mathrm{Ni}(\mathrm{COD})_{2}(136 \mathrm{mg}, 0.495 \mathrm{mmol})$, and tmeda $(74 \mu \mathrm{L}, 0.495 \mathrm{mmol})$ were stirred $3 \mathrm{~h}$ at $0^{\circ} \mathrm{C}$. Product 10b (55 mg, 52\%) was obtained as crystalline solid (mp 79-80 ${ }^{\circ} \mathrm{C}$ ) after chromatography (EtOAc/hexane 1/4). ${ }^{1} \mathrm{H}$ NMR (500 MHz, $\left.\mathrm{CDCl}_{3}\right) \delta 7.3-7.37(\mathrm{~m}, 4 \mathrm{H})$, $7.23-7.26(\mathrm{~m}, 1 \mathrm{H}), 3.08(\mathrm{ddd}, J=16.6,9.0,2.0 \mathrm{~Hz}, 1 \mathrm{H}), 2.44$ (sept, $J=4 \mathrm{~Hz}, 1 \mathrm{H}), 2.28$ (td, $J=16.3,2.3 \mathrm{~Hz}, 1 \mathrm{H}), 2.09-2.17(\mathrm{~m}, 1 \mathrm{H}), 1.89-1.93(\mathrm{~m}, 1 \mathrm{H}), 1.85(\mathrm{t}, J=2.0 \mathrm{~Hz}, 3 \mathrm{H})$, $1.73-1.80(\mathrm{~m}, 3 \mathrm{H}), 1.53-1.60(\mathrm{~m}, 1 \mathrm{H}), 1.43(\mathrm{~m}, 1 \mathrm{H}) ;{ }^{13} \mathrm{C}$ NMR (125 MHz, $\left.\mathrm{CDCl}_{3}\right)$ $\delta 138.2,137.0,136.9,128.3,128.0,127.0,98.5,48.5,42.2,38.8,35.1,25.8,11.4$; IR (film, $\mathrm{cm}^{-1}$ ) 3338; HRMS (EI) $\mathrm{m} / z$ calcd for $\mathrm{C}_{15} \mathrm{H}_{18} \mathrm{O}\left[\mathrm{M}^{+}\right] 214.1357$ found 214.1358. Anal. calcd for $\mathrm{C}_{15} \mathrm{H}_{18} \mathrm{O}$ : C, $83.66 \mathrm{H}, 8.90$ found $\mathrm{C}, 83.88 \mathrm{H}, 8.65$.

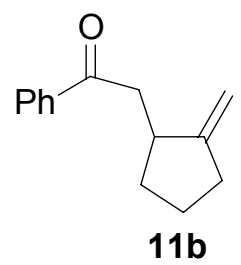

\section{Entry 10: 2-(2-Methylene-cyclopentyl)-1-phenyl-ethanone (11b)}

Following the general procedure, (2E)-1-phenyloct-2-en-7-yn-1-one (94 mg, $0.47 \mathrm{mmol})$, $\mathrm{Ni}(\mathrm{COD})_{2}(130 \mathrm{mg}, 0.47 \mathrm{mmol})$, and tmeda $(72 \mu \mathrm{L}, 0.47 \mathrm{mmol})$ were stirred for $3 \mathrm{~h}$ at 
$0^{\circ} \mathrm{C}$. Product 11b was obtained as a colorless oil after chromatography (EtOAc/Hex: 1/4) (25 mg, $0.23 \mathrm{mmol}, 27 \%$ ). \{Full spectroscopic details available in Montgomery, J.; Oblinger, E.; Savchenko, A. J. Am. Chem. Soc., 1997, 119(21), 4911.\}

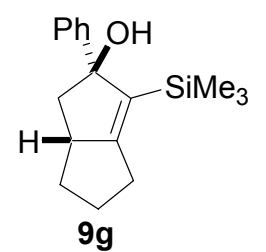

\section{Entry $11:\left(3 R^{*}, 5 R^{*}\right)-3-($ Phenyl)-2-(trimethylsilanyl)bicyclo[3.3.0]oct-1-en-3-ol (9g)}

Following the general procedure, $\mathrm{Ni}(\mathrm{COD})_{2}(20.0 \mathrm{mg}, 0.0727 \mathrm{mmol}$, 1eq.), tmeda (12 $\mu \mathrm{L}, 0.397 \mathrm{mmol}$, 1eq.) in $3 \mathrm{~mL} \mathrm{THF}$, and a solution of 1-phenyl-8-trimethylsilanyl-oct-2en-7-yn-1-one (19.9 $\mathrm{mg}, 0.0735 \mathrm{mmol})$ in $2 \mathrm{~mL}$ THF were stirred at $65^{\circ} \mathrm{C}$ for 14 hours at which point the red solution had become slightly turbid. The reaction was quenched with $7 \mathrm{~mL}$ anhydrous methanol. The reaction mixture was filtered through a pad of Celite and dried over anhydrous magnesium sulfate. Column chromatography (10:1 Hex/EtOAc) afforded a yellow oil, 9g (12.2 mg, $0.0448 \mathrm{mmol}, 61 \%) . \quad{ }^{1} \mathrm{H} \mathrm{NMR}\left(500 \mathrm{MHz}, \mathrm{CDCl}_{3}\right)$ : $\delta$ $7.42(\mathrm{~m}, 2 \mathrm{H}), 7.31(\mathrm{~m}, 2 \mathrm{H}), 7.21(\mathrm{~m}, 1 \mathrm{H}), 3.23(\mathrm{~m}, 1 \mathrm{H}), 2.25-2.43(\mathrm{~m}, 3 \mathrm{H}), 2.10-2.17(\mathrm{~m}$, 1H), 1.96-2.06 (m, 2H), $1.82(\mathrm{dd}, J=13.4,7.8 \mathrm{~Hz}, 1 \mathrm{H}), 1.79(\mathrm{~s}, 1 \mathrm{H}), 1.06-1.17(\mathrm{~m}, 1 \mathrm{H})$, -0.17 (s, 9H); ${ }^{13} \mathrm{C}$ NMR (125 MHz, $\left.\mathrm{CDCl}_{3}\right): \delta 172.9,148.0,135.6,127.8,126.5,126.0$, 97.8, 52.7, 51.5, 31.7, 28.9, 25.6, 0.02; IR (film, $\mathrm{cm}^{-1}$ ): 3474(br w), 1682(w), 1617(m), 1492(w), 1446(m), 1246(s), 835(vs), 758(s), 702(s); HRMS (EI) $m / z$ calcd for $\mathrm{C}_{17} \mathrm{H}_{24} \mathrm{OSi}$ $\left[\mathrm{M}^{+}\right] 272.1596$, found 272.1595 . 


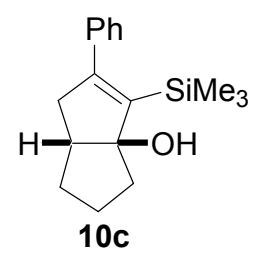

\section{Entry $12:\left(1 R^{*}, 5 S^{*}\right)-3-($ Phenyl)-2-(trimethylsilanyl)bicyclo[3.3.0]oct-2-en-1 (10c)}

A solution of $9 \mathrm{~g}(12.2 \mathrm{mg}, 0.0448 \mathrm{mmol})$ in $10 \mathrm{~mL} \mathrm{CH}_{2} \mathrm{Cl}_{2}$ and 1.0 gram silica gel was stirred for $8 \mathrm{~h}$ at room temperature. The mixture was filtered and the solvent removed in vacuo to give a quantitative yield of white solid 10c $(12.2 \mathrm{mg}, 0.0448 \mathrm{mmol}) . \quad{ }^{1} \mathrm{H} \mathrm{NMR}$ $\left(400 \mathrm{MHz}, \mathrm{CDCl}_{3}\right): \delta 7.26-7.32(\mathrm{~m}, 3 \mathrm{H}), 7.16(\mathrm{~m}, 2 \mathrm{H}), 3.06(\mathrm{dd}, J=17.6,8.8 \mathrm{~Hz}, 1 \mathrm{H})$, 2.47-2.41 (m, 1H), $2.28(\mathrm{dd}, J=17.8,4.2 \mathrm{~Hz}, 1 \mathrm{H}), 2.11$ (dquint, $J=6.4,1.6 \mathrm{~Hz}, 1 \mathrm{H}$ ), $1.92(\mathrm{~m}, 2 \mathrm{H}), 1.78(\mathrm{~m}, 1 \mathrm{H}), 1.68(\mathrm{sext}, J=6.4 \mathrm{~Hz}, 1 \mathrm{H}), 1.64(\mathrm{~s}, 1 \mathrm{H}), 1.35$ (sext, $J=6.8$ $\mathrm{Hz}, 1 \mathrm{H}),-0.03(\mathrm{~s}, 9 \mathrm{H}) ;{ }^{13} \mathrm{C} \mathrm{NMR}\left(100 \mathrm{MHz}, \mathrm{CDCl}_{3}\right): \delta 156.7,143.0,140.9,128.1,127.8$ 127.3, 102.6, 52.2, 47.7, 41.5, 34.7, 26.3, 1.2; IR $\left(\mathrm{CDCl}_{3}\right.$, film, $\left.\mathrm{cm}^{-1}\right): 3387(\mathrm{br} \mathrm{m})$, 1589(w), 1489(w), 1444(m), 1246(s), 836(vs), 758(s), 699(s); HRMS (EI) $m / z$ calcd for $\mathrm{C}_{17} \mathrm{H}_{24} \mathrm{OSi}\left[\mathrm{M}^{+}\right] 272.1596$, found 272.1597.

General Procedure for Table 2: (see general procedure above)

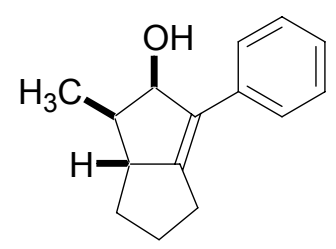

\section{Entry 1: (3R*,4R*,5R*)-4-Methyl-2-(phenyl)bicyclo[3.3.0]oct-1-en-3-ol}

Following the general procedure, 8-(phenyl)oct-2-en-7-ynal (47 mg, $0.236 \mathrm{mmol}$ ), $\mathrm{Ni}(\mathrm{COD})_{2}(65 \mathrm{mg}, 0.236 \mathrm{mmol}, 1$ eq.), and tmeda (36 $\mu \mathrm{L}, 0.236 \mathrm{mmol}, 1$ eq.) were stirred 
for $2 \mathrm{~h}$ at $0^{\circ} \mathrm{C}$. Methyl iodide $(44 \mu \mathrm{L}, 0.708 \mathrm{mmol}, 3$ eq.) was added dropwise and stirring at $0^{\circ} \mathrm{C}$ was continued $1 \mathrm{~h}$. Product $\{$ Table 2, Entry 1\} (34 mg, $0.16 \mathrm{mmol}, 68 \%$ ) was obtained as crystalline solid $\left(\mathrm{mp} 117-118^{\circ} \mathrm{C}\right)$ after chromatography (EtOAc/Hex, 1:9). ${ }^{1} \mathrm{H}$ NMR (500 MHz, $\left.\mathrm{CDCl}_{3}\right) \delta 7.50(\mathrm{~d}, J=8.0 \mathrm{~Hz}, 2 \mathrm{H}), 7.34-7.37(\mathrm{~m}, 2 \mathrm{H}), 7.20-$ $7.23(\mathrm{~m}, 1 \mathrm{H}), 5.17(\mathrm{~d}, J=5.5 \mathrm{~Hz}, 1 \mathrm{H}), 2.86-2.91(\mathrm{~m}, 1 \mathrm{H}), 2.55-2.61(\mathrm{~m}, 1 \mathrm{H}), 2.39-2.47$ (m, 1H), 2.11-2.17 (m, 1H), 1.98-2.06 (m, 2H), 1.92 (sext, $J=7.0 \mathrm{~Hz}, 1 \mathrm{H}), 1.44$ (br s, $1 \mathrm{H}), 1.21(\mathrm{~d}, J=7.0 \mathrm{~Hz}, 3 \mathrm{H}), 0.99-1.09(\mathrm{~m}, 1 \mathrm{H}) ;{ }^{13} \mathrm{C} \mathrm{NMR}\left(125 \mathrm{MHz}, \mathrm{CDCl}_{3}\right): \delta 156.3$, 135.7, 132.5, 128.7, 127.0, 126.6, 84.9, 58.9, 45.3, 30.6, 29.7, 26.5, 13.5; IR (film, $\mathrm{cm}^{-1}$ ) 3364; HRMS (EI) $m / z$ calcd for $\mathrm{C}_{15} \mathrm{H}_{18} \mathrm{O}\left[\mathrm{M}^{+}\right]$214.1357, found 214.1354. Irradiation of the methine signal at $\delta 5.17(\mathrm{C}-\mathrm{H}-\mathrm{OH})$ resulted in $6.7 \%$ NOE enhancement to the methine signal at $\delta 1.89-1.93\left(\underline{\mathrm{CH}}-\mathrm{CH}_{3}\right)$. Irradiation of the methyl signal at $\delta 1.21$ resulted in a $2.5 \% \mathrm{NOE}$ enhancement to the methine signal at $\delta 2.86-2.91 \mathrm{ppm}$ (bridgehead-CH).

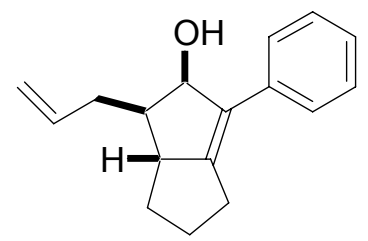

\section{Entry 2: 1-Allyl-3-phenyl-1,2,4,5,6,6a-hexahydro-pentalen-2-ol}

Following the general procedure, 8-(phenyl)oct-2-en-7ynal (100 mg, $0.505 \mathrm{mmol})$, $\mathrm{Ni}(\mathrm{COD})_{2}(140 \mathrm{mg}, 0.505 \mathrm{mmol})$ and tmeda $(76 \mu \mathrm{L}, 0.50 \mathrm{mmol})$ were stirred for $2 \mathrm{~h}$ at $0^{\circ} \mathrm{C}$. Allyl iodide $(139 \mu \mathrm{L}, 1.52 \mathrm{mmol})$ was added dropwise at $0^{\circ} \mathrm{C}$ and the reaction mixture was stirred at the same temperature for 2 hours. Product \{Table 2, Entry 2$\}$ (87 $\mathrm{mg}, 0.363 \mathrm{mmol}, 72 \%)$ was obtained as a white crystalline solid $\left(\mathrm{mp} 97-98^{\circ} \mathrm{C}\right)$ after chromatography (EtOAc/Hex, 1:5). ${ }^{1} \mathrm{H}$ NMR $\left(400 \mathrm{MHz}, \mathrm{CDCl}_{3}\right) \delta 7.50(\mathrm{~d}, J=8.0 \mathrm{~Hz}$, 2H), $7.36(\mathrm{t}, J=7.8 \mathrm{~Hz}, 2 \mathrm{H}), 7.23(\mathrm{q}, J=7.0 \mathrm{~Hz}, 1 \mathrm{H}), 5.96(\mathrm{ddt}, J=17.4,10.0,7.2 \mathrm{~Hz}$, 
$1 \mathrm{H}), 5.28(\mathrm{~d}, J=5.6 \mathrm{~Hz}, 1 \mathrm{H}), 5.15-5.20(\mathrm{~m}, 1 \mathrm{H}), 5.04-5.07(\mathrm{~m}, 1 \mathrm{H}), 2.91-2.97(\mathrm{~m}, 1 \mathrm{H})$, 2.33-2.61 (m, 4H), 1.96-2.18 (m, 3H), 1.81-1.92 (m, 1H), $1.60(\mathrm{~s}, 1 \mathrm{H}), 0.99-1.11(\mathrm{~m}$, $1 \mathrm{H}) ;{ }^{13} \mathrm{C}$ NMR $\left(100 \mathrm{MHz}, \mathrm{CDCl}_{3}\right): \delta 156.2,138.4,135.6,132.1,128.7,127.0,126.7$, 115.6, 83.6, 57.5, 50.3, 33.9, 31.4, 29.7, 26.3. IR (film, $\mathrm{cm}^{-1}$ ) 3364, 3213, 1498, 1444, 1089, 1036, 909, 770, 690; HRMS (EI) $m / z$ calcd for $\mathrm{C}_{17} \mathrm{H}_{20} \mathrm{O}\left[\mathrm{M}^{+}\right]$240.1514, found 240.1513. Irradiation of the methine signal at $\delta 5.28(\mathrm{C} \underline{\mathrm{H}}-\mathrm{OH})$ resulted in $4.88 \% \mathrm{NOE}$ enhancement to the methine signal at $\delta$ 1.81-1.92 $\left(\mathrm{C} \underline{\mathrm{H}}-\mathrm{CH}_{2} \mathrm{CHCH}_{2}\right)$.

\section{Experimental Scheme A: Towards product Table 2, Entry 3}

$\mathrm{Ni}(\text { acac })_{2}+2.5 \mathrm{DIBAL}+4 \mathrm{COD} \underset{-78^{0} \mathrm{C} \rightarrow 0^{0} \mathrm{C}}{\stackrel{\mathrm{THF}}{\longrightarrow}} \mathrm{Ni(COD)_{2 }}$

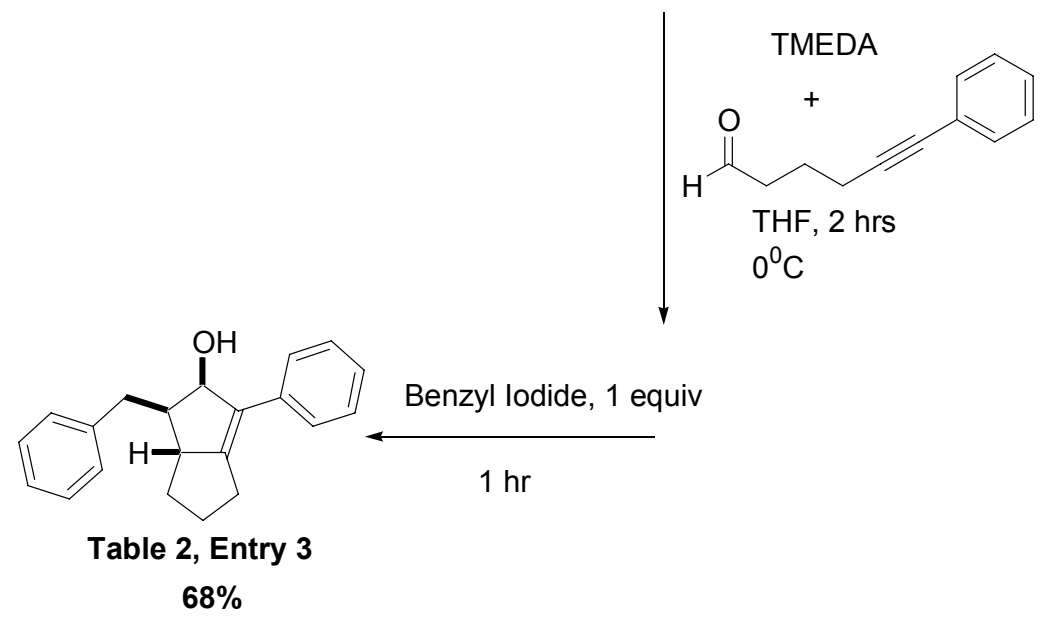

\section{Entry 3 : $\left(3 R^{*}, 4 R^{*}, 5 R *\right)-2-P h e n y l-4-\left[\right.$ benzyl]-bicyclo[3.3.0]oct-1-en-3-ol: ${ }^{2}$}

(This is also a representative procedure for Scheme 8 in paper.)

In a round bottom flask under vacuum $\mathrm{Ni}(\mathrm{acac})_{2}(104 \mathrm{mg}, 0.404 \mathrm{mmol})$ was heated briefly with a heat gun. After cooling and establishing a positive argon atmosphere, $1 \mathrm{~mL}$ THF

\footnotetext{
${ }^{2}$ See leading reference Mackenzie, P.; Krysan, D.J., J. Org. Chem. 1990, 55, 4229.
} 
was added, followed by the addition of COD $(198 \mu \mathrm{L}, 1.62 \mathrm{mmol})$. The solution was then cooled to $-78^{\circ} \mathrm{C}$ in a dry ice/acetone bath to give a green slurry and DIBAL-H (1.0 M/THF, $1.01 \mathrm{mmol}, 1.01 \mathrm{~mL}$ ) was introduced over one hour using a syringe pump to give a reddish brown solution which was allowed to warm to $0^{\circ} \mathrm{C}$ over a $1 \mathrm{hr}$ period. THF was removed under vacuum and the resulting yellowish brown solid was washed with $1 \mathrm{~mL}$ portions of cold ether until the brown residues were removed to give a yellow solid. THF (12 mL) was added, followed by the addition of tmeda ( $38 \mu \mathrm{L}, 0.25 \mathrm{mmol})$, and the solution was stirred for $5 \mathrm{~min}$ at rt. 8-(Phenyl)oct-2-en-7ynal (50 $\mathrm{mg}, 0.25 \mathrm{mmol}$ ) as a solution in $3 \mathrm{~mL}$ THF was introduced via a cannula at $0^{\circ} \mathrm{C}$. After 2 hours at $0^{\circ} \mathrm{C}$, benzyl iodide (100 $\mu \mathrm{L}, 0.757 \mathrm{mmol})$ was added and the reaction mixture was kept at the same temperature for another hour. The reaction was quenched with aqueous saturated sodium bicarbonate, extracted 3 times with ethyl acetate and dried over magnesium sulfate. After removal of solvent in vacuo the crude material was subjected to $\mathrm{SiO}_{2}$ chromatography (EtOAc/Hex, 1:10). The product \{Table 2, Entry 3\} (50 mg, 68\% mp 99-100 ${ }^{\circ} \mathrm{C}$ ) and was obtained as a white crystalline solid). ${ }^{1} \mathrm{H}$ NMR $\left(400 \mathrm{MHz}, \mathrm{CDCl}_{3}\right)$ : $\delta 7.47(\mathrm{~d}, J=8.0 \mathrm{~Hz}, 2 \mathrm{H}), 7.31-7.36(\mathrm{~m}, 6 \mathrm{H}), 7.19-7.26(\mathrm{~m}, 2 \mathrm{H}), 5.23(\mathrm{~d}, J=6.0 \mathrm{~Hz}$, 1H), 2.97-3.1 (m, 2H), $2.88(\mathrm{dd}, J=13.4,8.0 \mathrm{~Hz}, 1 \mathrm{H}), 2.56(\mathrm{dd}, J=18.4,10.4 \mathrm{~Hz}, 1 \mathrm{H})$, 2.35-2.44 (m, 1H), 2.08- 2.20 (m, 1H), 1.89-2.07 (m, 2H), 1.55-1.61 (m, 2H), 0.85 (dq, $J$ $=12.4,8.0 \mathrm{~Hz}, 1 \mathrm{H}) ;{ }^{13} \mathrm{C} \mathrm{NMR}\left(100 \mathrm{MHz}, \mathrm{CDCl}_{3}\right) \delta 156.3141 .8,135.5,132.2,129.2$, 128.7, 128.6, 127.0, 126.7, 126.1, 83.5, 57.3, 52.7, 35.5, 31.1, 29.6, 26.3; IR (film, $\mathrm{cm}^{-1}$ ) 3323.7; HRMS (EI) $m / z$ calcd for $\mathrm{C}_{21} \mathrm{H}_{22} \mathrm{O}\left[\mathrm{M}^{+}\right]$290.1671, found 290.1668. Irradiation of the methine signal at $\delta 5.23(\mathrm{C} \underline{\mathrm{H}}-\mathrm{OH})$ resulted in $7.8 \%$ NOE enhancement to the methine signal at $\delta 2.08-2.20\left(\right.$ C $\left.\underline{H}-\mathrm{CH}_{2}-\mathrm{Ph}\right)$. 


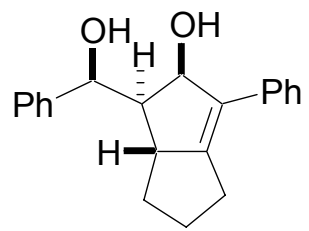

Entry 4: $\left(3 R^{*}, 4 R *, 5 R *\right)-2-P h e n y l-4-\left[\left(S^{*}\right)\right.$-phenlymethanol]bicyclo[3.3.0]oct-1-en-3ol

Following the general procedure, 8-(phenyl)oct-2-en-7-ynal (47 mg, $0.236 \mathrm{mmol}$ ), $\mathrm{Ni}(\mathrm{COD})_{2}(65 \mathrm{mg}, 0.236 \mathrm{mmol}, 1$ eq.), and tmeda (36 $\mu \mathrm{L}, 0.236 \mathrm{mmol}, 1$ eq.) were stirred for $2 \mathrm{~h}$ at $0^{\circ} \mathrm{C}$. Benzaldehyde $\left(72 \mu \mathrm{L}, 0.708 \mathrm{mmol}, 3\right.$ eq.) was added dropwise at $0^{\circ} \mathrm{C}$ and the reaction mixture was stirred at $\mathrm{rt}$ for $12 \mathrm{~h}$. Product \{Table 2, Entry 4$\}$ (59 mg, 0.193 mmol, $82 \%$ ) was obtained as crystalline solid ( $\left.\mathrm{mp} 133-134^{\circ} \mathrm{C}\right)$ after chromatography (EtOAc/Hex, 1:6). ${ }^{1} \mathrm{H}$ NMR $\left(400 \mathrm{MHz}, \mathrm{CDCl}_{3}\right) \delta 7.44(\mathrm{t}, J=6.4 \mathrm{~Hz}, 4 \mathrm{H}), 7.36(\mathrm{t}, J=$ $7.2 \mathrm{~Hz}, 4 \mathrm{H}), 7.21-7.28(\mathrm{~m}, 2 \mathrm{H}), 5.50(\mathrm{~d}, J=5.6 \mathrm{~Hz}, 1 \mathrm{H}), 5.29(\mathrm{~d}, J=4.0 \mathrm{~Hz}, 1 \mathrm{H}), 3.93$ (br s, 1H), 3.48-3.54 (m, 1H), $2.56(\mathrm{dt}, J=18.4,5.6,1 \mathrm{H}), 2.28-2.39$ (m, 2H), 2.14-2.19 $(\mathrm{m}, 1 \mathrm{H}), 1.92-2.00(\mathrm{~m}, 2 \mathrm{H}), 1.37-1.44(\mathrm{~m}, 1 \mathrm{H}), 0.61-0.72(\mathrm{~m}, 1 \mathrm{H}) ;{ }^{13} \mathrm{C}$ NMR $(100 \mathrm{MHz}$ $\left.\mathrm{CDCl}_{3}\right): \delta 157.6,144.0,134.9,131.3,128.9,128.4,127.2,127.0,126.9,125.9,85.1,73.3$ 56.9, 51.4, 31.8, 29.4, 25.9; IR (film, $\mathrm{cm}^{-1}$ ) 3356; HRMS (EI) $m / z$ calcd for $\mathrm{C}_{21} \mathrm{H}_{22} \mathrm{O}\left[\mathrm{M}^{+}\right.$] 306.1698, found $288.1514\left[\mathrm{M}-\mathrm{H}_{2} \mathrm{O}\right]^{+}$. The stereochemical assignment was confirmed by single crystal X-ray analysis. 


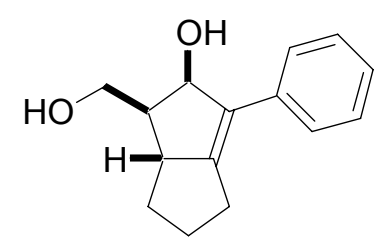

Entry 5: $\quad(3 R *, 4 R *, 5 R *)-2-P h e n y l-4-[h y d r o x y m e t h y l] b i c y c l o[3.3 .0]$ oct-1-en-3-ol; Following the general procedure, 8-(phenyl)oct-2-en-7-ynal (50 mg, $0.25 \mathrm{mmol}$ ), $\mathrm{Ni}(\mathrm{COD})_{2}(70 \mathrm{mg}, 0.25 \mathrm{mmol})$ and tmeda $(38 \mu \mathrm{L}, 0.25 \mathrm{mmol})$ were stirred for $2 \mathrm{~h}$ at $0^{\circ} \mathrm{C}$. A suspension of paraformaldehyde $(68 \mathrm{mg}, 0.76 \mathrm{mmol})$ in $3 \mathrm{~mL}$ ether was added dropwise at $0^{\circ} \mathrm{C}$ and the reaction mixture was stirred at the same temperature for 2 hours. The product \{Table 2, Entry 5\} (46 mg, $0.20 \mathrm{mmol}, 80 \%)$ was obtained as a white crystalline solid (mp 132-133을 after chromatography (EtOAc/Hex, 4:1). ${ }^{1} \mathrm{H}$ NMR (400 $\left.\mathrm{MHz}, \mathrm{CDCl}_{3}\right) \delta 7.46(\mathrm{~d}, J=7.2 \mathrm{~Hz}, 2 \mathrm{H}), 7.36(\mathrm{t}, J=8.4 \mathrm{~Hz}, 2 \mathrm{H}), 7.22(\mathrm{t}, J=7.2 \mathrm{~Hz}$, $1 \mathrm{H}), 5.51(\mathrm{~d}, J=6.4 \mathrm{~Hz}, 1 \mathrm{H}), 3.86-3.98(\mathrm{~m}, 2 \mathrm{H}), 3.11-3.17(\mathrm{~m}, 1 \mathrm{H}), 2.37-3.08(\mathrm{br} \mathrm{s}, 1 \mathrm{H})$, $2.59(\mathrm{dd}, J=18.8,10.4,1 \mathrm{H}), 2.37-2.47(\mathrm{~m}, 1 \mathrm{H}), 1.98-2.17(\mathrm{~m}, 4 \mathrm{H}), 0.99-1.11(\mathrm{~m}, 1 \mathrm{H})$; ${ }^{13} \mathrm{C} \mathrm{NMR}\left(100 \mathrm{MHz}, \mathrm{CDCl}_{3}\right) \delta 156.6,135.1,131.5,128.9,127.0,126.9,84.1,62.4,53.3$, 50.9, 30.9, 29.5, 26.2; IR (film, $\mathrm{cm}^{-1}$ ) 3333(br), 1712, 1272, 1204, 1119, 1020, 756, 692; HRMS (EI) $m / z$ calcd for $\mathrm{C}_{15} \mathrm{H}_{18} \mathrm{O}_{2}\left[\mathrm{M}^{+}\right] 230.1307$, found 230.1307. Irradiation of the methine signal at $\delta 5.54(\mathrm{C} \underline{\mathrm{H}}-\mathrm{OH})$ resulted in $6.9 \%$ NOE enhancement to the methine signal at 1.99-2.18 $\left(\underline{\mathrm{C}}-\underline{\mathrm{C}} \mathrm{CH}_{2} \mathrm{OH}\right)$. Irradiation of the bridgehead methine at $3.15-3.21$ resulted in $1.8 \%$ NOE enhancement to the $\mathrm{C}_{2} \mathrm{OH}$ signal at 3.91-4.02. 


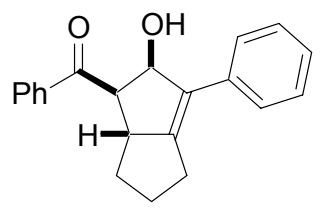

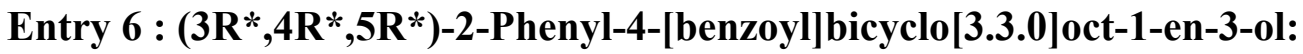

Following the general procedure, 8-(phenyl)oct-2-en-7ynal (100 mg, $0.505 \mathrm{mmol}$ ), $\mathrm{Ni}(\mathrm{COD})_{2}(140 \mathrm{mg}, 0.505 \mathrm{mmol})$ and tmeda $(76 \mu \mathrm{L}, 0.505 \mathrm{mmol})$ were stirred for $2 \mathrm{~h}$ at $0^{\circ} \mathrm{C}$. Benzoyl chloride $(59 \mu \mathrm{L}, 0.505 \mathrm{mmol})$ was added dropwise at $0^{\circ} \mathrm{C}$ and the reaction mixture was stirred at the same temperature for 10 min. The product $\{$ Table 2, Entry 6$\}$ was obtained as a white crystalline solid (111 mg, $0.3651 \mathrm{mmol}, 72 \%, \mathrm{mp} 126-127^{\circ} \mathrm{C}$ ) after chromatography (EtOAc/Hex, 1:5). ${ }^{1} \mathrm{H}$ NMR (400 MHz, $\left.\mathrm{CDCl}_{3}\right) \delta 8.05(\mathrm{~d}, J=7.2$ $\mathrm{Hz}, 2 \mathrm{H}), 7.59-7.62(\mathrm{~m}, 1 \mathrm{H}), 7.52(\mathrm{t}, J=7.2 \mathrm{~Hz}, 4 \mathrm{H}), 7.36(\mathrm{t}, J=7.8 \mathrm{~Hz}, 2 \mathrm{H}), 7.21-7.26$ $(\mathrm{m}, 1 \mathrm{H}), 5.75(\mathrm{t}, J=7.0 \mathrm{~Hz}, 1 \mathrm{H}), 3.90-3.97(\mathrm{~m}, 1 \mathrm{H}), 3.84(\mathrm{t}, J=6.8 \mathrm{~Hz}, 1 \mathrm{H}), 2.66(\mathrm{dd}, J$ $=17.8,9.8 \mathrm{~Hz}, 1 \mathrm{H}), 2.46-2.55(\mathrm{~m}, 1 \mathrm{H}), 2.42(\mathrm{~d}, J=8.4 \mathrm{~Hz}, 1 \mathrm{H}), 2.02-2.23(\mathrm{~m}, 3 \mathrm{H}), 1.09-$ $1.20(\mathrm{~m}, 1 \mathrm{H}) ;{ }^{13} \mathrm{C}$ NMR $\left(100 \mathrm{MHz}, \mathrm{CDCl}_{3}\right) \delta 200.1,155.1,137.4,134.8,133.5,130.0$ 129.0, 128.8, 128.5, 127.0, 127.0, 84.7, 57.3, 53.1, 31.3, 29.5, 26.2; IR (film, cm ${ }^{-1}$ ) 3458, 1679,1596, 1496, 1446, 1252, 1020; HRMS (EI) $m / z$ calcd for $\mathrm{C}_{21} \mathrm{H}_{20} \mathrm{O}_{2}[\mathrm{M}]^{+}$304.1463, found $286.1358\left[\mathrm{M}-\mathrm{H}_{2} \mathrm{O}\right]^{+}$. Irradiation of the methine signal at $\delta 5.75(\mathrm{C} \underline{\mathrm{H}}-\mathrm{OH})$ resulted in $8.3 \%$ NOE enhancement to the methine signal at $\delta 3.84(\mathrm{CH}-\mathrm{CO}-\mathrm{Ph})$.

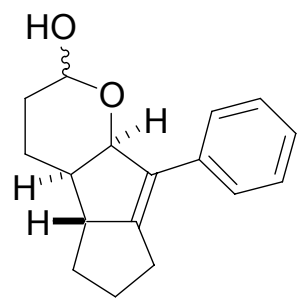

Entry 7: 8-Phenyl-2,3,3a,3b,4,5,6,7a-octahydro-1H-7-oxa-cyclopenta[a]inden-6-ol 
Following the general procedure, 8-(phenyl)oct-2-en-7ynal $(50 \mathrm{mg}, 0.25 \mathrm{mmol})$, $\mathrm{Ni}(\mathrm{COD})_{2}(70 \mathrm{mg}, 0.25 \mathrm{mmol})$ and tmeda $(38 \mu \mathrm{L}, 0.25 \mathrm{mmol})$ were stirred for $2 \mathrm{~h}$ at $0^{\circ} \mathrm{C}$. Acrolein $(51 \mu \mathrm{L}, 0.76 \mathrm{mmol})$ was added dropwise at $0^{\circ} \mathrm{C}$ and the reaction mixture was stirred at the same temperature for 2 hours. The product \{Table 2, Entry 7$\}$ (47 mg, 0.18 mmol, $72 \%$ ) was obtained as a white crystalline solid (mp 124-125 ${ }^{\circ} \mathrm{C}$ ) after chromatography (EtOAc/Hexane, 1:5) as a 3:2 inseparable mixture of diastereomers. ${ }^{1} \mathrm{H}$ NMR (400 MHz, $\left.\mathrm{CDCl}_{3}\right) \delta$ 7.46-7.48 (m, 2H), $7.33(\mathrm{t}, J=7.7 \mathrm{~Hz}, 2 \mathrm{H}), 7.17-7.20(\mathrm{~m}$, $1 \mathrm{H}), 5.47(\mathrm{~d}, J=5.5 \mathrm{~Hz}, 0.6 \mathrm{H}), 5.33-5.34(\mathrm{~m}, 0.6 \mathrm{H}), 5.17(\mathrm{~d}, J=4.0 \mathrm{~Hz}, 0.4 \mathrm{H}), 4.76(\mathrm{t}, J$ $=7.5 \mathrm{~Hz}, 0.4 \mathrm{H}), 3.2-3.26(\mathrm{~m}, 0.4 \mathrm{H}), 3.12-3.17(\mathrm{~m}, 0.6 \mathrm{H}), 2.88(\mathrm{~m}, 0.4 \mathrm{H}), 2.79(\mathrm{~m}, 0.6 \mathrm{H})$, 2.58-2.64 (m, 1H), 2.41-2.51 (m, 1H), 2.12-2.20 (m, 2H), 1.73-2.10 (m, 4H), 1.51-1.64 (m, 2H), 1.02-1.10 (m,1H); ${ }^{13} \mathrm{C}$ NMR (100 MHz, $\left.\mathrm{CDCl}_{3}\right) \delta$ 157.9, 157.7, 135.6, 130.1, $129.8,128.63,128.61,126.93,126.87,126.6,126.5,95.6,92.4,88.0,80.4,57.1,54.8,43$. 7, 43.5, 30.8, 30.5, 30.0, 29.7, 29.2, 27.0, 26.7, 26.5, 23.2, 20.1; IR(film, $\left.\mathrm{cm}^{-1}\right) \quad 3386$, 1708, 1496, 1444, 1008; HRMS (EI) $m / z$ calcd for $\mathrm{C}_{17} \mathrm{H}_{20} \mathrm{O}_{2}\left[\mathrm{M}^{+}\right]$256.1463, found 256.1458 . 


\section{Experimental Scheme B: Synthesis of precursor 17 for Scheme 5}
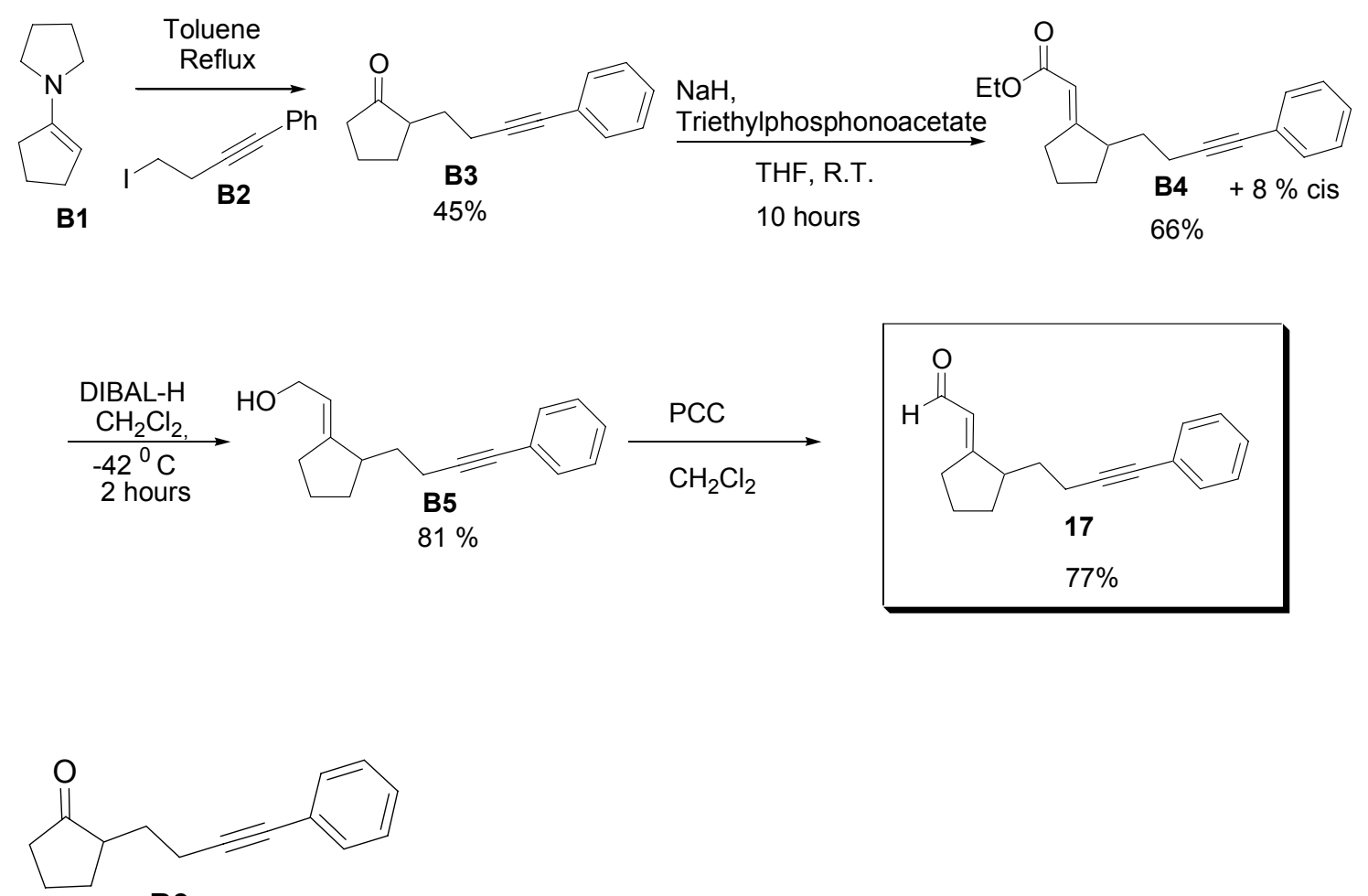

B3

\section{B3, 2-(4-Phenyl-but-3-ynyl)-cyclopentanone: ${ }^{3}$}

The iodide B2 (9.145 g, $35.72 \mathrm{mmol})$ was added to a solution of the pyrrolidine enamine of cyclopentanone B1 (5.872 g, $42.86 \mathrm{mmol}, 1.2$ eq. $)^{4}$ in dry toluene (86 mL) and the reaction mixture was heated to reflux under argon for $18 \mathrm{~h}$. Water was added $(10 \mathrm{~mL})$, and the mixture was heated to reflux for an additional $30 \mathrm{~min}$, cooled, poured into cold $10 \%$ aqueous $\mathrm{HCl}$ and extracted $3 \mathrm{x}$ with ethyl acetate. The organic layer was washed with brine, dried over $\mathrm{MgSO}_{4}$, and filtered. Evaporation of the solvent and purification by silica gel column chromatography (EtOAc:Hex, 1:8) gave $3.4 \mathrm{~g}(16 \mathrm{mmol}, 45 \%)$ of B3 as a colorless oil. ${ }^{1} \mathrm{H}$ NMR $\left(400 \mathrm{MHz}, \mathrm{CDCl}_{3}\right) \delta$ 7.35-7.39 (m, 2H), 7.26-7.29 (m, $3 \mathrm{H}), 2.52(\mathrm{qt}, J=17.2,6.4 \mathrm{~Hz}, 2 \mathrm{H}), 2.24-2.37(\mathrm{~m}, 3 \mathrm{H}), 2.00-2.19(\mathrm{~m}, 3 \mathrm{H}), 1.75-1.87(\mathrm{~m}$,

\footnotetext{
${ }^{3}$ See leading reference Ghera, E., Maurya, R.; Ben-David, Y., J. Org. Chem., 1988, 53, 1912.

${ }^{4}$ Preparation of B1 done as in Murray, D.F.; Baum, M.W.; Jones, M., J. Org. Chem., 1986, 51, 1.
} 
1H), 1.52-1.62 (m, 2H); ${ }^{13} \mathrm{C} \mathrm{NMR}\left(100 \mathrm{MHz}, \mathrm{CDCl}_{3}\right) \delta 222.1,131.7,128.4,127.9,124.0$, 89.5, 81.5, 48.5, 38.3, 29.7, 28.9, 21.0, 17.9 IR (film, $\left.\mathrm{cm}^{-1}\right)$ 1735, 1490, 1202, 1156, 1070, 755, 692; HRMS (EI) $m / z$ calcd for $\mathrm{C}_{15} \mathrm{H}_{16} \mathrm{O}\left[\mathrm{M}^{+}\right]$212.1201, found 212.1197.

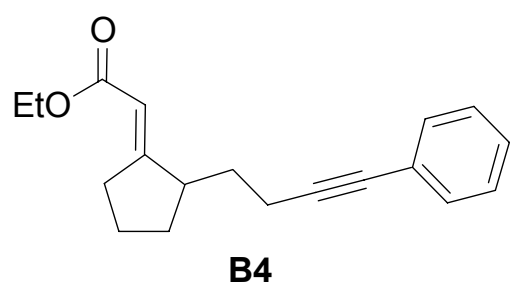

\section{B4, [2-(4-Phenyl-but-3-ynyl)-cyclopentylidene]-acetic acid ethyl ester: ${ }^{5}$}

$\mathrm{NaH}(60 \%$ dispersion in mineral oil, 33mg, $0.84 \mathrm{mmol})$ was washed with hexanes, and the resulting powder was suspended in dry THF $(2 \mathrm{~mL})$ and cooled in a dry ice/acetone bath. Triethylphosphonoacetate $(231 \mu \mathrm{L}, 1.17 \mathrm{mmol})$ in $2 \mathrm{~mL}$ THF was added dropwise and the reaction mixture was stirred for $30 \mathrm{~min}$ at $\mathrm{rt}$. The mixture was cooled in a dry ice/acetone bath and a solution of 2-(4-phenyl-but-3-ynyl)-cyclopentanone in $1 \mathrm{~mL}$ THF was added dropwise. The mixture was allowed to warm to rt while stirring over $10 \mathrm{~h}$. The reaction mixture was quenched with saturated ammonium chloride and extracted $3 \mathrm{x}$ with ether. The combined ether extract was washed with brine and the organic layer dried over anhydrous $\mathrm{MgSO}_{4}$. Evaporation of the solvent and purification by column chromatography (EtOAc:Hex, 1:10) furnished $\quad E$-[2-(4-phenyl-but-3-ynyl)cyclopentylidene]-acetic acid ethyl ester $\mathbf{B} 4$ as a colorless oil $(132 \mathrm{mg}, 0.468 \mathrm{mmol}$, 66\%) along with Z-[2-(4-phenyl-but-3-ynyl)-cyclopentylidene]-acetic acid ethyl ester (15 mg, $0.053 \mathrm{mmol}, 8 \%)$. Product $\mathbf{B} 4{ }^{1} \mathrm{H}$ NMR $\left(400 \mathrm{MHz}, \mathrm{CDCl}_{3}\right) \delta$ 7.37-7.40 (m, 2H), 7.25-7.30 (m, 3H), 5.77 (q, $J=2.4 \mathrm{~Hz}, 1 \mathrm{H}), 4.15$ (q, $J=7.6 \mathrm{~Hz}, 2 \mathrm{H}), 2.95-3.01(\mathrm{~m}, 1 \mathrm{H})$,

\footnotetext{
${ }^{5}$ See leading reference Srikrishna, A.; Kumar, P.P., Tetrahedron, 2000, 56(41), 8189.
} 
2.66-2.78 (m, 2H), 2.39-2.56 (m, 2H), 1.91-2.03 (m, 2H), 1.80-1.90 (m, 1H), 1.54-1.68 (m, 2H), 1.32-1.36 (m, 1H), $1.27(\mathrm{t}, J=7.4 \mathrm{~Hz}, 3 \mathrm{H}) ;{ }^{13} \mathrm{C}$ NMR $\left(100 \mathrm{MHz}, \mathrm{CDCl}_{3}\right)$ $\delta 171.5,167.2,131.8,128.4,127.9,124.0,112.0,89.7,81.4,59.8,46.0,33.2,32.8,31.5$, 24.4, 17.9, 14.6; IR (film, $\mathrm{cm}^{-1}$ ) 3390(br w), 1713(s), 1275, 1201, 1037, 758, 693; HRMS (EI) $m / z$ calcd for $\mathrm{C}_{19} \mathrm{H}_{22} \mathrm{O}_{2}\left[\mathrm{M}^{+}\right]$282.1620, found 282.1612.

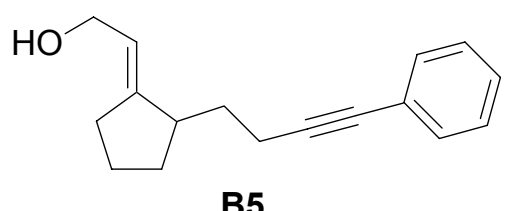

\section{B5, 2-[2-(4-Phenyl-but-3-ynyl)-cyclopentylidene]-ethanol:}

A solution of [2-(4-phenyl-but-3-ynyl)-cyclopentylidene]-acetic acid ethyl ester (295 mg,

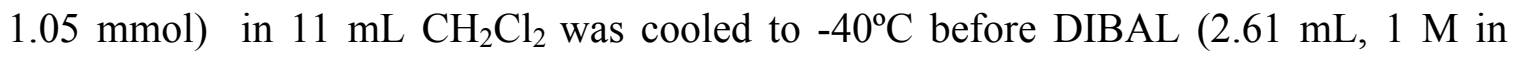
hexanes, $2.62 \mathrm{mmol}$ ) was introduced dropwise. The mixture was kept at this temperature for 30 minutes followed by the dropwise addition of $0.5 \mathrm{~mL}$ anhydrous methanol (CAUTION: vigorous evolution of gas). The mixture was allowed to warm to $\mathrm{rt}$ and was stirred with an aqueous saturated solution of sodium potassium tartarate overnight. The organic fraction was extracted $3 \times$ with ethyl acetate and dried over anhydrous magnesium sulfate. After solvent removal by rotary evaporation, column chromatography (1:3, Hex/EtOAc) afforded B5, 2-[2-(4-Phenyl-but-3-ynyl)cyclopentylidene]-ethanol, (204 mg, $0.850 \mathrm{mmol}, 81 \%)$ as a colorless oil. ${ }^{1} \mathrm{H}$ NMR (400 $\left.\mathrm{MHz}, \mathrm{CDCl}_{3}\right) \delta$ 7.36-7.40 (m, 2H), 7.26-7.30 (m, 3H), 5.47-5.50 (m, 1H), $4.15(\mathrm{~d}, J=7.2$ $\mathrm{Hz}, 2 \mathrm{H}), 2.35-2.55(\mathrm{~m}, 4 \mathrm{H}), 2.22-2.30(\mathrm{~m}, 1 \mathrm{H}), 1.87-1.99(\mathrm{~m}, 2 \mathrm{H}), 1.73-1.82(\mathrm{~m}, 1 \mathrm{H})$, 1.47-1.63 (m, 3H), 1.24-1.33 (m, 1H); ${ }^{13} \mathrm{C}$ NMR $\left(100 \mathrm{MHz}, \mathrm{CDCl}_{3}\right) \delta 150.4,131.8$ 128.4, 127.8, 124.2, 119.6, 90.4, 81.0, 61.1, 43.8, 33.5, 32.3, 29.2, 24.3, 18.0: IR (film, 
$\mathrm{cm}^{-1}$ ) 3342, 1490, 999, 755, 692; HRMS (EI) $\mathrm{m} / z$ calcd for $\mathrm{C}_{17} \mathrm{H}_{20} \mathrm{O}\left[\mathrm{M}^{+}\right]$240.1514, found 240.1506 .

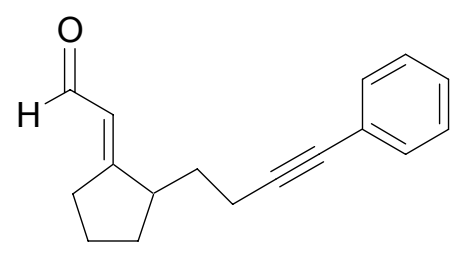

17

\section{7, [2-(4-Phenyl-but-3-ynyl)-cyclopentylidene]-acetaldehyde:}

A solution of 2-[2-(4-phenyl-but-3-ynyl)-cyclopentylidene]-ethanol (85mg, $0.35 \mathrm{mmol})$ in $9 \mathrm{~mL} \mathrm{CH} \mathrm{Cl}_{2}$ was stirred while PCC (122mg, $0.567 \mathrm{mmol}, 1.6$ equiv.) was added portion wise. The reaction mixture stirred for $2 \mathrm{~h}$ at $\mathrm{rt}$. The solvent was removed by rotary evaporation, and the crude material was loaded on a silica gel pad. After elution with $\mathrm{CH}_{2} \mathrm{Cl}_{2}$, product $\mathbf{B 5}$ (65mg, $0.27 \mathrm{mmol}, 77 \%$ ) was isolated as a colorless oil. ${ }^{1} \mathrm{H}$ NMR (400 MHz, $\left.\mathrm{CDCl}_{3}\right) \delta 9.90(\mathrm{~d}, J=8.0 \mathrm{~Hz}, 1 \mathrm{H}), 7.37-7.39(\mathrm{~m}, 2 \mathrm{H}), 7.26-7.31(\mathrm{~m}$, $3 \mathrm{H})$, 5.98-6.00 (m, $1 \mathrm{H}), 2.97-3.02(\mathrm{~m}, 1 \mathrm{H}), 2.72-2.81(\mathrm{~m}, 2 \mathrm{H}), 2.42-2.59(\mathrm{~m}, 2 \mathrm{H}), 1.91-$ 2.11(m, 3H), 1.57-1.77 (m, 2H), 1.35-1.44 (m, 1H); $\left.{ }^{13} \mathrm{C} \mathrm{NMR} \mathrm{(100} \mathrm{MHz,} \mathrm{CDCl}_{3}\right) \delta$ $192.1,176.1,131.8,128.5,128.0,123.9,123.1,89.3,81.6,45.9,32.5,31.1,30.8,24.5$, 17.9; IR (film, $\mathrm{cm}^{-1}$ ) 1677, 1490, 1274, 1162, 756, 692; HRMS (EI) $\mathrm{m} / z$ calcd for $\mathrm{C}_{17} \mathrm{H}_{18} \mathrm{O}\left[\mathrm{M}^{+}\right]$238.1358, found 238.1357.

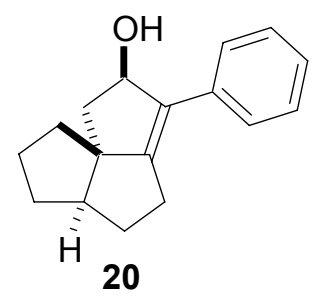

20, 3-Phenyl-1,2,4,5,5a,6,7,8-octahydro-cyclopenta[c]pentalen-2-ol: 
Following the general procedure, [2-(4-phenyl-but-3-ynyl)-cyclopentylidene]acetaldehyde (100 mg, $0.420 \mathrm{mmol}), \mathrm{Ni}(\mathrm{COD})_{2}(116 \mathrm{mg}, 0.420 \mathrm{mmol})$ and tmeda (64 $\mu \mathrm{L}$, $0.40 \mathrm{mmol})$ were stirred for $2 \mathrm{~h}$ at room temperature. Anhydrous methanol $(2 \mathrm{~mL})$ was added and the reaction mixture was stirred for another $15 \mathrm{~min}$. Product $15(72 \mathrm{mg}, 0.30$ mmol, $71 \%$ ) was obtained as a white crystalline solid $\left(\mathrm{mp} 77-78^{\circ} \mathrm{C}\right)$ after column chromatography (EtOAc/Hex, 1:5). ${ }^{1} \mathrm{H}$ NMR $\left(500 \mathrm{MHz}, \mathrm{CDCl}_{3}\right) \delta 7.45(\mathrm{~d}, J=7.5 \mathrm{~Hz}$, 2H) 7.36 (t, $J=7.5 \mathrm{~Hz}, 2 \mathrm{H}), 7.24$ (q, $J=7.5 \mathrm{~Hz}, 1 \mathrm{H}), 5.43(\mathrm{~d}, J=6.5 \mathrm{~Hz}, 1 \mathrm{H}), 2.45-2.55$ (m, 2H), 1.95-2.12 (m, 4H), 1.77-1.92 (m, 4H), 1.63-1.71 (m, 3H), 1.40-1.48 (m, 1H); ${ }^{13} \mathrm{C}$ NMR $\left(125 \mathrm{MHz}, \mathrm{CDCl}_{3}\right) \delta 158.9,135.2,131.7,128.7,127.6,126.9,81.9,66.8,48.4$, 47.3, 42.0, 36.0, 34.9, 27.6, 25.6; IR (film, $\mathrm{cm}^{-1}$ ) 3327, 1650, 1598, 1496, 1445, 1012; HRMS (EI) $m / z$ calcd for $\mathrm{C}_{17} \mathrm{H}_{20} \mathrm{O}\left[\mathrm{M}^{+}\right] 240.1514$, found 240.1514. The structural assignment was confirmed by single crystal X-Ray analysis.
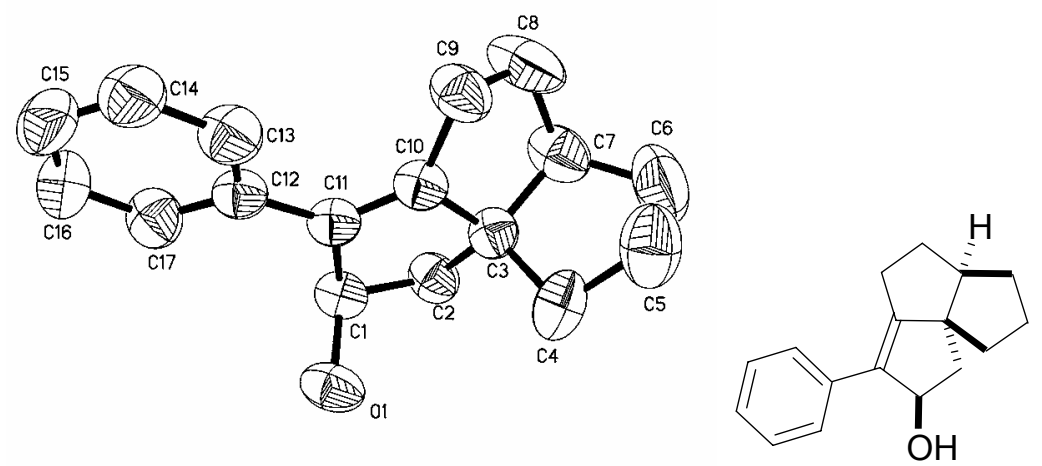

Figure 1: ORTEP perspective views of 20 showing the labeling system. Protons were removed for clarity. Thermal ellipsoids are drawn at a $30 \%$ probability level. 


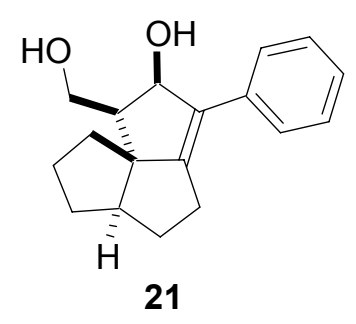

\section{1, 1-Hydroxymethyl-3-phenyl-1,2,4,5,5a,6,7,8-octahydro-cyclopenta[c]pentalen-2- ol:}

Following the general procedure, [2-(4-phenyl-but-3-ynyl)-cyclopentylidene]acetaldehyde $(71 \mathrm{mg}, 0.30 \mathrm{mmol}), \mathrm{Ni}(\mathrm{COD})_{2}(82 \mathrm{mg}, 0.30 \mathrm{mmol})$ and tmeda $(45 \mu \mathrm{L}, 0.30$ $\mathrm{mmol})$ were stirred for $2 \mathrm{~h}$ at room temperature. Paraformaldehyde ( $81 \mathrm{mg}, 0.89 \mathrm{mmol})$ as a slurry in ether was added dropwise and the reaction mixture was stirred at room temperature for another 4 hours. After chromatography (EtOAc/Hex, 1:5) 21 was obtained as a white crystalline solid (37 mg, $0.14 \mathrm{mmol}, 46 \%) .{ }^{1} \mathrm{H}$ NMR (400 MHz, $\left.\mathrm{CDCl}_{3}\right) \delta 7.44(\mathrm{~d}, J=7.2 \mathrm{~Hz}, 2 \mathrm{H}), 7.35(\mathrm{t}, J=7.8 \mathrm{~Hz}, 2 \mathrm{H}), 7.21-7.26(\mathrm{~m}, 1 \mathrm{H}), 5.41(\mathrm{~d}, J$ $=5.6,1 \mathrm{H}), 4.09(\mathrm{t}, J=10.2 \mathrm{~Hz}, 1 \mathrm{H}), 3.86(\mathrm{dd}, J=10.8,6.0 \mathrm{~Hz}, 1 \mathrm{H}), 2.49(\mathrm{dd}, J=9.2$, $5.6 \mathrm{~Hz}, 2 \mathrm{H}), 2.11-2.22(\mathrm{~m}, 2 \mathrm{H}), 1.89-2.05(\mathrm{~m}, 4 \mathrm{H}), 1.66-1.80(\mathrm{~m}, 3 \mathrm{H}), 1.49-1.61(\mathrm{~m}, 2 \mathrm{H})$, 1.39-1.46 (m, 1H); ${ }^{13} \mathrm{C}$ NMR $\left(100 \mathrm{MHz}, \mathrm{CDCl}_{3}\right) \delta 160.4,135.1,131.5,128.8,127.6$, 127.0, 81.2, 66.9, 60.5, 54.8, 47.0, 36.1, 35.7, 34.9, 27.6, 25.8; IR (film, $\mathrm{cm}^{-1}$ ) 3343, 1493, 1445, 1014, 690; HRMS (EI) $m / z$ calcd for $\mathrm{C}_{18} \mathrm{H}_{22} \mathrm{O}_{2}\left[\mathrm{M}^{+}\right]$270.1620, found 270.1620. Irradiation of the methine signal at $\delta 5.41(\mathrm{C} \underline{\mathrm{H}}-\mathrm{OH})$ resulted in $7.6 \% \mathrm{NOE}$ enhancement to the methine signal at $\delta 2.11-2.22\left(\mathrm{C} \underline{\mathrm{H}}-\mathrm{CH}_{2} \mathrm{OH}\right)$.

\section{Experimental Scheme C: Towards compounds leading up to 22 (Scheme 6)}



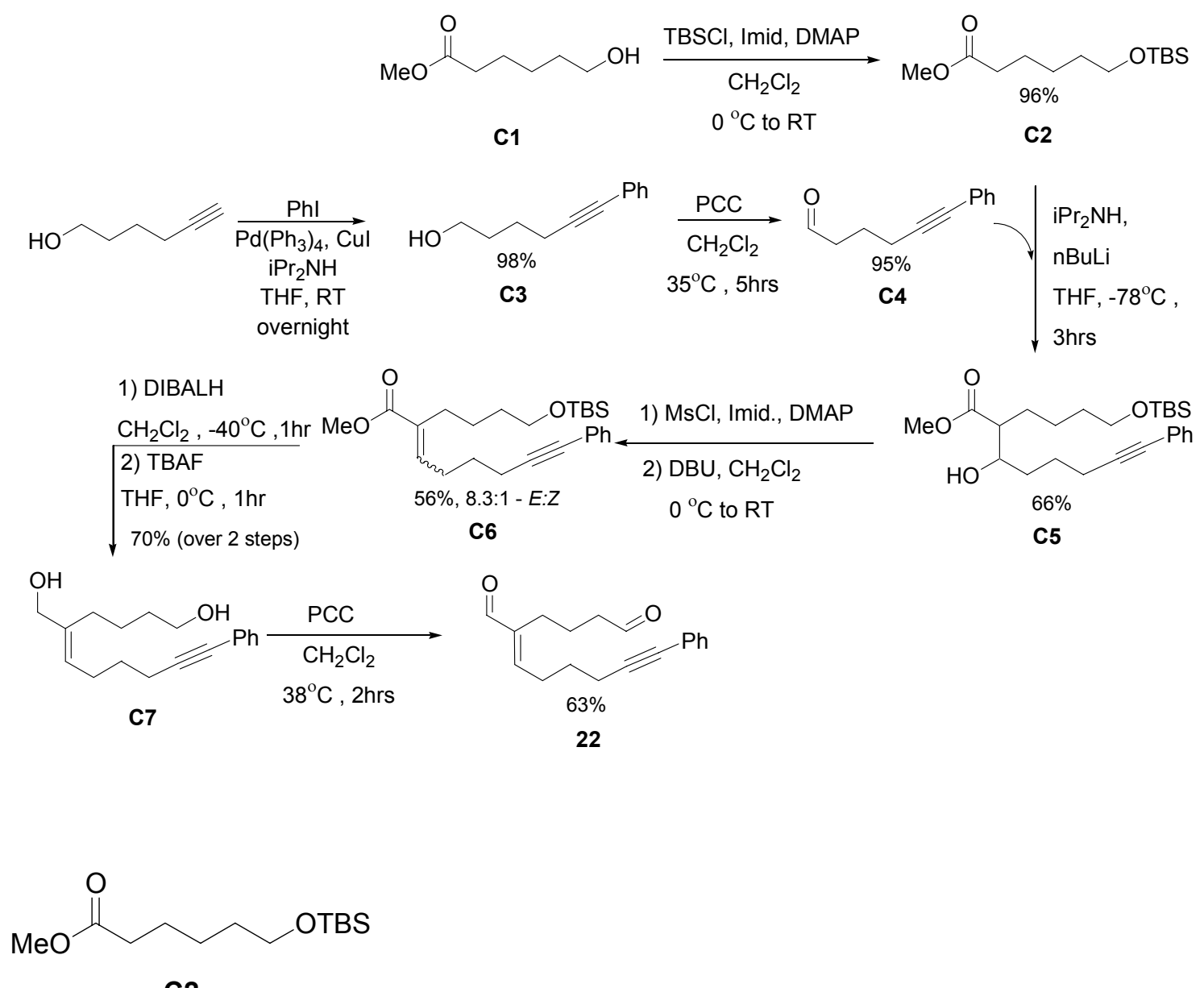

C2

C2, 6-(tert-Butyl-dimethyl-silanyloxy)-hexanoate: ${ }^{6}$ A solution of C1 (2.55 g, 17.4 $\mathrm{mmol})$ in $10 \mathrm{~mL}$ dichloromethane was added to a mixture of imidazole $(2.03 \mathrm{~g}, 29.8$ mmol, 1.7 eq.) and DMAP (215 mg, $1.76 \mathrm{mmol}, 0.1$ eq.) in $15 \mathrm{~mL}$ of freshly distilled dichloromethane and stirred at $0^{\circ} \mathrm{C}$. Five minutes later, a solution of $\mathrm{TBSCl}(3.91 \mathrm{~g}, 25.9$ mmol, 1.5 eq.) in $10 \mathrm{~mL}$ dichloromethane was added dropwise via a syringe over $10 \mathrm{~min}$. The mixture was allowed to warm to rt over 3.5 hours. The reaction was quenched with $3 \times 10 \mathrm{~mL}$ brine, extracted with dichloromethane, and dried over $\mathrm{MgSO}_{4}$. The solvent was removed by rotary evaporation to yield C2 as a colorless oil (4.34 g, $16.7 \mathrm{mmol}$, 96\%). ${ }^{1} \mathrm{H}$ NMR (400 MHz, $\left.\mathrm{CDCl}_{3}\right) \delta 3.65(\mathrm{~s}, 3 \mathrm{H}), 3.59(\mathrm{t}, J=6.4 \mathrm{~Hz}, 2 \mathrm{H}), 2.30(\mathrm{t}, J=$

\footnotetext{
${ }^{6}$ For leading reference see Fujiwara, K.; Amano, A.; Tokiwano, T.; Murai, A., Tetrahedron, 2000, 56(8), 1065 .
} 
$8.0 \mathrm{~Hz}, 2 \mathrm{H}$ ), 1.63 (quint, $J=7.6 \mathrm{~Hz}, 2 \mathrm{H}$ ), 1.52 (quint, $J=6.4 \mathrm{~Hz}, 2 \mathrm{H}$ ), 1.31-1.39 (m, 2H), 0.87 (s, 9H), $0.03(\mathrm{~s}, 6 \mathrm{H}) ;{ }^{13} \mathrm{C} \mathrm{NMR} \delta 174.4,63.1,51.6,34.3,32.7,26.2,25.6,25.0$, 18.5, -5.1; IR (film, $\left.\mathrm{cm}^{-1}\right): 2929(\mathrm{~m}), 2856(\mathrm{~m}), 1741(\mathrm{~s}), 1462(\mathrm{~m}), 1436(\mathrm{~m}), 1388(\mathrm{w})$, 1361(m), 1253(s), 1200(m), 1163(m), 1097(vs), 1006(m), 830(vs), 772(vs), 663(s); HRMS (EI) $m / z$ calcd for $\mathrm{C}_{13} \mathrm{H}_{28} \mathrm{O}_{3} \mathrm{Si} \quad 245.1573$, found: $245.1575\left[\mathrm{M}-\mathrm{CH}_{3}\right]^{+}$.

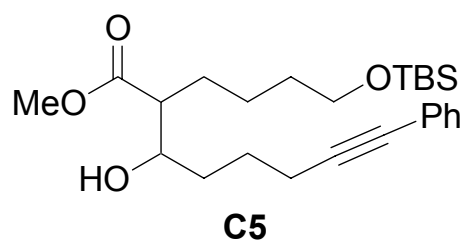

C5, Methyl 2-[4-(tert-Butyl-dimethyl-silanyloxy)-butyl]-3-hydroxy-7-phenyl-hept-6ynoate: To a $0^{\circ} \mathrm{C}$ solution of diisopropylamine $(1.60 \mathrm{~mL}, 11.4 \mathrm{mmol}, 1.3$ eq. $)$ in $20 \mathrm{~mL}$ THF was added of n-butyl lithium (7.10 mL of $1.6 \mathrm{M}$ in hexanes, $11.4 \mathrm{mmol}, 1.3 \mathrm{eq}$.$) ,$ and the mixture was stirred at this temperature for $30 \mathrm{~min}$. The mixture was then cooled to $-78^{\circ} \mathrm{C}$ for $20 \mathrm{~min}$ before adding a solution of $\mathbf{C 2}(2.26 \mathrm{~g}, 8.68 \mathrm{mmol}, 1$ eq.) in $3 \mathrm{~mL}$ THF via syringe dropwise the most reproducible results were obtained using a syringe drive at $0.35 \mathrm{~mL} / \mathrm{min}\}$. The mixture was stirred at this temperature for 35 minutes, stirred outside of the cold bath at room temperature for 1 minute, and placed back in the cold bath. A solution of $\mathbf{C 4}(1.52 \mathrm{~g}, 8.78 \mathrm{mmol}, 1$ eq.) in $5 \mathrm{~mL}$ THF was added via a syringe dropwise \{the most reproducible results were obtained using a syringe drive at $0.20 \mathrm{~mL} / \mathrm{min}\}$ and the mixture stirred at $-78^{\circ} \mathrm{C}$ for 3 more hours and TLC analysis $(10: 1$ hexanes/ethyl acetate) shows the reaction was complete. The reaction was quenched with $25 \mathrm{~mL}$ of $\mathrm{pH} 8$ buffer $\left(\mathrm{NH}_{4} \mathrm{Cl} / \mathrm{NH}_{4} \mathrm{OH}\right)$ at $-78^{\circ} \mathrm{C}$ and the mixture warmed to ambient temperature. The organic material was extracted with ether and dried over magnesium 
sulfate. Column chromatography (hexanes:ethyl acetate, 10:1) allowed for the clean separation of a yellow oil, as a mixture of isomers of $\mathbf{C 5}(2.47 \mathrm{~g}, 5.71 \mathrm{mmol}, 66 \%) .{ }^{1} \mathrm{H}$ NMR (500 MHz, $\left.\mathrm{CDCl}_{3}\right): \delta$ 7.37-7.39 (m, 2H), 7.25-7.27 (m, 3H), 3.69 and $3.70(\mathrm{~s}, 3 \mathrm{H})$, $3.58(\mathrm{dt}, J=6.4,2.4 \mathrm{~Hz}, 2 \mathrm{H}), 2.43-2.55(\mathrm{~m}, 4 \mathrm{H}), 1.44-1.86(\mathrm{~m}, 9 \mathrm{H}), 1.22-1.43(\mathrm{~m}, 2 \mathrm{H})$, 0.88 (s, 9H), $0.03(\mathrm{~s}, 6 \mathrm{H}) ;{ }^{13} \mathrm{C} \mathrm{NMR}\left(125 \mathrm{MHz}, \mathrm{CDCl}_{3}\right): \delta$ 176.4, 176.1, 131.7, 128.4, $127.8,124.1,89.9,81.3,72.1,71.8,63.0,51.9,51.8,51.3,51.2,34.9,33.6,32.94,32.86$ 29.7, 27.0, 26.2, 25.3, 25.2, 24.3, 24.0, 19.4, 18.5, -5.07; IR ( $\mathrm{CDCl}_{3}$, film, $\left.\mathrm{cm}^{-1}\right): 3456(\mathrm{br}$ w), 2928(m), 2856(m), 1732(m), 1716(m), 1599(w), 1490(w) 1462(m), 1435(m), 1387(w), 1360(w), 1253(s), 1198(m), 1162(m), 1096(s), 1006(m), 976(m), 833(vs), 754(vs), 691(vs); HRMS (EI) $m / z$ calcd for $\mathrm{C}_{24} \mathrm{H}_{40} \mathrm{O}_{4} \mathrm{Si}\left[\mathrm{M}^{+}\right]$432.2696, found: 432.2693 .

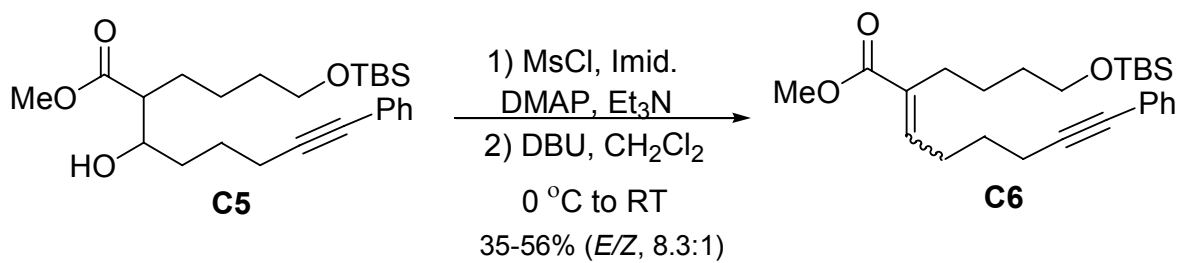

\section{C6, Methyl 2-[4-(tert-Butyl-dimethyl-silanyloxy)-butyl]-7-phenyl-hept-2-en-6-} ynoate:

A $15 \mathrm{~mL}$ round bottom flask was charged with DMAP (11 mg, 0.098mmol, 0.14eq.), 1 $\mathrm{mL}$ dichloromethane, and triethylamine $(400 \mu \mathrm{L}, 2.87 \mathrm{mmol}, 4$ eq. $)$ and cooled to $0^{\circ} \mathrm{C}$. Five minutes later, a solution of $\mathbf{C 5}(307 \mathrm{mg}, 0.710 \mathrm{mmol}$, 1 eq. $)$ in $2 \mathrm{~mL}$ dichloromethane was cannula transferred into the mixture. After five more minutes, $\mathrm{MsCl}(130 \mu \mathrm{L}, 2.87 \mathrm{mmol}, 4$ eq.) was added which made the mixture turbid and yellow. The mixture was stirred one additional hour to allow the ice bath to reach ambient temperature. The mixture was poured into $30 \mathrm{~mL}$ of water and the organic layer extracted with ether. The ether layer was washed with brine and followed by the addition 
of and dried over magnesium sulfate. The yellow residue was taken up in $2 \mathrm{~mL}$ of dichloromethane and stirred at room temperature while DBU $(450 \mu \mathrm{L}, 2.99 \mathrm{mmol}, 4.2$ eq.) was added dropwise over 2 minutes. The reaction was quenched after 2 hours with $4 \mathrm{~mL}$ of $1 \mathrm{~N} \mathrm{HCl}$, washed with $\mathrm{NaHCO}_{3}$ and brine; the organic layer dried over magnesium sulfate. Column chromatography (5:1 hexanes/ether) allowed for the clean separation of a colorless oil, C6 (140 mg, $0.338 \mathrm{mmol}, 56 \%$; 8.3:1 E:Z, $125 \mathrm{mg}$ of $E) .{ }^{1} \mathrm{H}$ NMR (400 MHz, $\left.\mathrm{CDCl}_{3}\right): \delta 7.38(\mathrm{~m}, 2 \mathrm{H}), 7.26-7.29(\mathrm{~m}, 3 \mathrm{H}), 6.77(\mathrm{t}, J=7.2 \mathrm{~Hz}, 1 \mathrm{H})$, $3.72(\mathrm{~s}, 3 \mathrm{H}), 3.58$ (t, $J=5.6 \mathrm{~Hz}, 2 \mathrm{H}), 2.45$ (t, $J=7.2 \mathrm{~Hz}, 2 \mathrm{H}$ ), 2.36 (quint., $J=7.2 \mathrm{~Hz}$, 2H), 1.75 (quint., $J=7.2 \mathrm{~Hz}, 2 \mathrm{H}), 1.40-1.56(\mathrm{~m}, 4 \mathrm{H}), 0.88(\mathrm{~s}, 9 \mathrm{H}), 0.03(\mathrm{~s}, 6 \mathrm{H}) ;{ }^{13} \mathrm{C}$ NMR (DEPT, $\left.100 \mathrm{MHz}, \mathrm{CDCl}_{3}\right): \delta 168.6\left(\mathrm{C}_{\text {quat. }}\right), 141.9(\mathrm{CH}), 133.3\left(\mathrm{C}_{\text {quat. }}\right), 131.7(\mathrm{CH})$, 128.4(CH), 127.9(CH), 124.0( $\left.\mathrm{C}_{\text {quat. }}\right), 89.5\left(\mathrm{C}_{\text {quat. }}\right), 81.5\left(\mathrm{C}_{\text {quat. }}\right), 63.2\left(\mathrm{CH}_{2}\right), 51.9\left(\mathrm{CH}_{3}\right)$, 32.9 $\left(\mathrm{CH}_{2}\right), \quad 28.1\left(\mathrm{CH}_{2}\right), \quad 27.9\left(\mathrm{CH}_{2}\right), \quad 26.9\left(\mathrm{CH}_{2}\right), \quad 26.2\left(\mathrm{CH}_{3}\right), \quad 25.9\left(\mathrm{CH}_{2}\right), \quad 19.3\left(\mathrm{CH}_{3}\right)$, 18.5( $\left.\mathrm{C}_{\text {quat. }}\right),-5.04\left(\mathrm{CH}_{3}\right)$; IR (film, $\left.\mathrm{cm}^{-1}\right): 2949(\mathrm{~m}), 2928(\mathrm{~m}), 2858(\mathrm{~m}), 1713(\mathrm{~s}), 1644(\mathrm{w})$, 1598(w), 1490(m), 1461(m), 1434(m), 1387(w), 1360(w), 1280(m), 1253(s), 1193(m), 1096(vs), 1006(m), 835(vs), 775(s), 755(vs), 691(s); HRMS (EI) $\mathrm{m} / \mathrm{z}$ calcd for $\mathrm{C}_{25} \mathrm{H}_{37} \mathrm{O}_{3} \mathrm{Si}\left[\mathrm{M}^{+}\right]$413.2512, found 413.2150.

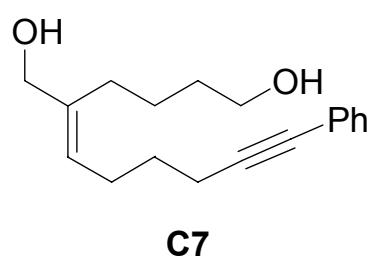

\section{C7, 2-(5-Phenyl-pent-4-ynylidene)-hexane-1,6-diol:}

A $15 \mathrm{~mL}$ round bottom flask was charged with $\mathbf{C 6}$ (530 mg of only the $E$ isomer, 1.28 mmol, 1eq.) and $6 \mathrm{~mL}$ dichloromethane and then cooled to $-40^{\circ} \mathrm{C}$ (dry ice in acetonitrile 
bath). DIBALH (4.0 mL of a 1.0M in hexanes, $4.0 \mathrm{mmol}, 3.1$ eq.) was added via syringe and the mixture stirred 1 hour. TLC analysis (hexanes-ether, 5:1) indicated the first step of this reaction was complete. The reaction was quenched with $1 \mathrm{~mL}$ methanol and the reaction mixture poured into a 1:1 mixture of ethyl acetate to saturated potassium sodium tartrate and extracted with ether. The organic fraction was dried over magnesium sulfate and the solvent was removed by rotary evaporation. The crude colorless oil was subsequently taken up in $10 \mathrm{~mL}$ of THF and chilled to $0^{\circ} \mathrm{C}$. The TBS group was removed with the addition of TBAF (2.2 $\mathrm{mL}$ of $1.0 \mathrm{M}$ in THF, $2.2 \mathrm{mmol}, 1.7$ eq.) and stirring for 1 hour while allowing the bath to warm to room temperature. The reaction mixture was quenched with $3 \mathrm{~mL}$ of water and the organic material extracted with ether and dried over magnesium sulfate. Column chromatography $(5 \%$ methanol in dichloromethane) afforded the clean separation of a colorless oil, C7 (244 mg, 0.895 mmol, $70 \%$ over both steps). This compound was spectroscopically identical to the product obtain in a defined two step sequence (below).

This transformation was also done in step-wise fashion thereby confirming the combination of steps:
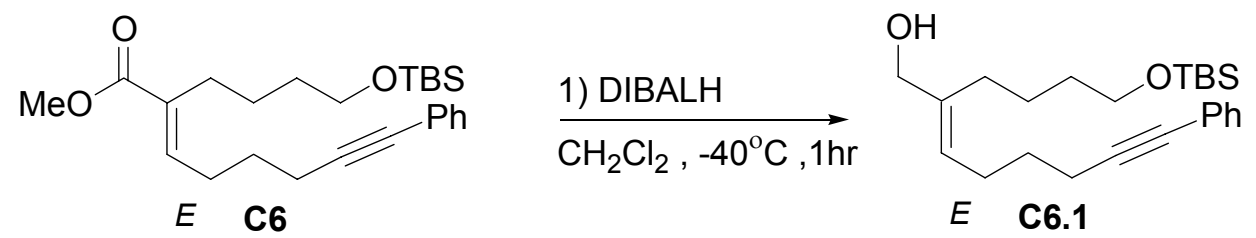

\section{C6.1, 2-[4-(tert-Butyl-dimethyl-silanyloxy)-butyl]-7-phenyl-hept-2-en-6-yn-1-ol:}

A $15 \mathrm{~mL}$ round bottom flask was charged with $\mathbf{C 6}(78 \mathrm{mg}, 0.19 \mathrm{mmol}$, 1eq.) and $6 \mathrm{~mL}$ dichloromethane and then cooled to $-40^{\circ} \mathrm{C}$ (dry ice in acetonitrile bath). DIBALH (600 
$\mu \mathrm{L}$ of a $1.0 \mathrm{M}$ in hexanes, $4.0 \mathrm{mmol}, 3.1$ eq.) was added via syringe and the mixture stirred 1 hour. TLC analysis (5:1 hexanes/ether,) indicated this reaction was complete. The reaction was quenched with $1 \mathrm{~mL}$ methanol and the reaction mixture poured into a 1:1 mixture of ethyl acetate to saturated potassium sodium tartrate and extracted with ether. The aqueous layer was washed with brine and the residual organic material extracted with ether. The organic fraction was dried over magnesium sulfate and the solvent removed on the rotary evaporator to give a colorless oil, C6.1, (47 mg, 0.12 mmol, 67\% yield). ${ }^{1} \mathrm{H}$ NMR (500 MHz, $\left.\mathrm{CDCl}_{3}\right): \delta 7.38(\mathrm{~m}, 2 \mathrm{H}), 7.26(\mathrm{~m}, 3 \mathrm{H}), 5.44(\mathrm{t}, J$ $=7.5 \mathrm{~Hz}, 1 \mathrm{H}), 4.05(\mathrm{~s}, 2 \mathrm{H}), 3.59(\mathrm{t}, J=6.0 \mathrm{~Hz}, 2 \mathrm{H}) ,2.42(\mathrm{t}, J=7.0 \mathrm{~Hz}, 2 \mathrm{H}), 2.22(\mathrm{q}, J=$ $7.5 \mathrm{~Hz}, 2 \mathrm{H}), 2.14$ (t, $J=7.5 \mathrm{~Hz}, 2 \mathrm{H}), 1.68$ (quint., $J=7.0 \mathrm{~Hz}, 2 \mathrm{H}$ ), 1.51 (br m, 2H), 1.45 (br m, 2H), 1.37 (br s, 1H), $0.88(\mathrm{~s}, 9 \mathrm{H}), 0.03(\mathrm{~s}, 6 \mathrm{H}) ;{ }^{13} \mathrm{C} \mathrm{NMR}\left(125 \mathrm{MHz}, \mathrm{CDCl}_{3}\right): \delta$ $140.2,131.7,128.4,127.7,126.1,124.2,90.2,81.1,67.3,63.2,33.1,28.9,28.1,26.8$, 26.2, 25.1, 19.2, 18.5, -5.0; IR ( $\mathrm{CDCl}_{3}$, film, $\left.\mathrm{cm}^{-1}\right): 3366(\mathrm{w}), 2939(\mathrm{~s}), 2857(\mathrm{~s}), 1490(\mathrm{~m})$, $1472(\mathrm{~m}), 1462(\mathrm{~m}), 1387(\mathrm{w}), 1360(\mathrm{w}), 1254(\mathrm{~m}), 1099(\mathrm{~s}), 1005(\mathrm{~m}), 835(\mathrm{vs}), 775(\mathrm{~s})$, 755(s), 691(m); HRMS (EI) $m / z$ calcd for $\mathrm{C}_{24} \mathrm{H}_{38} \mathrm{O}_{2} \mathrm{Si}\left[\mathrm{M}^{+}\right]$386.2641, found 386.2638. Followed by removal of the TBS group with TBAF:

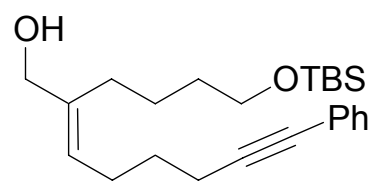

C6.1

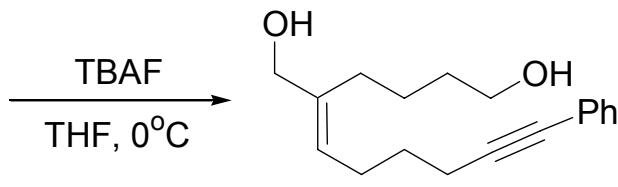

C7

A solution of C6.1 (47 mg, $0.12 \mathrm{mmol}, 1$ eq.) in $4 \mathrm{~mL}$ of THF was chilled to $0^{\circ} \mathrm{C}$ and treated with TBAF $(150 \mu \mathrm{L}$ of $1.0 \mathrm{M}$ in THF, $0.150 \mathrm{mmol}, 1.2$ eq.) with stirring for 1 hour at $0^{\circ} \mathrm{C}$ followed by 30 minutes at room temperature. The reaction mixture was quenched with $2 \mathrm{~mL}$ of water and the organic material extracted with ether and dried over magnesium sulfate. Column chromatography (5\% methanol in dichloromethane) afforded 
a colorless oil, $\mathbf{C} 7(20 \mathrm{mg}, 0.075 \mathrm{mmol}, 62 \%) .{ }^{1} \mathrm{H}$ NMR $\left(500 \mathrm{MHz}, \mathrm{CDCl}_{3}\right): \delta 7.39(\mathrm{~m}$, 2H), $7.27(\mathrm{~m}, 3 \mathrm{H}), 5.45(\mathrm{t}, J=7.6 \mathrm{~Hz}, 1 \mathrm{H}), 4.05(\mathrm{~s}, 2 \mathrm{H}), 3.62(\mathrm{t}, J=6.0 \mathrm{~Hz}, 2 \mathrm{H}), 2.42(\mathrm{t}$, $J=7.2 \mathrm{~Hz}, 2 \mathrm{H}$ ), 2.23 (q, $J=7.2 \mathrm{~Hz}, 2 \mathrm{H}), 2.16$ (t, $J=8.4 \mathrm{~Hz}, 2 \mathrm{H}$ ), 1.67 (quint., $J=7.2$ $\mathrm{Hz}, 2 \mathrm{H}), 1.47-1.61(\mathrm{~m}, 5 \mathrm{H}), 1.37(\mathrm{~m}, 2 \mathrm{H}) ;{ }^{13} \mathrm{C} \mathrm{NMR}\left(125 \mathrm{MHz}, \mathrm{CDCl}_{3}\right): \delta 140.0,131.7$, $128.5,127.8,126.4,124.2,90.2,81.1,67.4,62.9,32.9,28.9,28.0,26.8,24.9,19.2$; IR $\left(\mathrm{CDCl}_{3}\right.$, film, $\left.\mathrm{cm}^{-1}\right)$ : 3334(br, m), 2931(s), 2861(m), 1733 (w), 1598 (w), $1490(\mathrm{~m}), 1457$

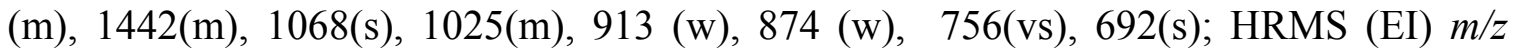
calcd for $\mathrm{C}_{18} \mathrm{H}_{24} \mathrm{O}_{2}[\mathrm{M}+\mathrm{H}]^{+}$272.1776, found 272.1779.

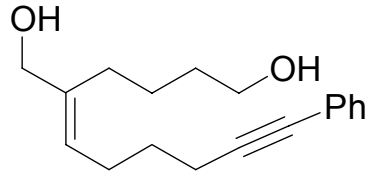

C7

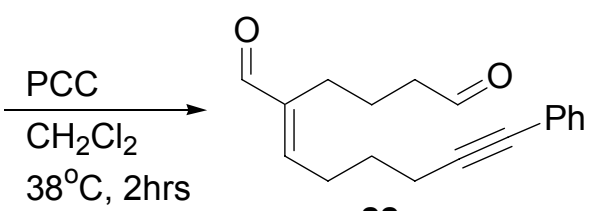

22

\section{2, 2-(5-Phenyl-pent-4-ynylidene)-hexane-1,6-dial:}

A solution of PCC (490 mg, $2.27 \mathrm{mmol}, 4.5$ eq.) in $10 \mathrm{~mL}$ dichloromethane was stirred under a nitrogen atmosphere for 5 minutes before a solution of $\mathbf{C} 7(136 \mathrm{mg}, 0.499 \mathrm{mmol}$, 1 eq.) in $10 \mathrm{~mL}$ dichlormethane was cannula transferred in. The reaction was stirred at $\mathrm{rt}$ for 2 hours before being concentrated in vacuo to about $5 \mathrm{~mL}$. The black reaction mixture was heated to $40^{\circ} \mathrm{C}$ then poured through a wide silica gel pad which was pre wetted with a $1 \% \mathrm{MeOH}$ in dichlormethane. With a slight vacuum, about $300 \mathrm{~mL}$ of the eluent (1\% MeOH in dichloromethane) helped push through the product (Caution: only the colorless material is collected.). The solvent is removed on the rotary evaporator and then on the vacuum pump to give a pure, colorless oil $22(84.3 \mathrm{mg}, 0.314 \mathrm{mmol}, 63 \%$ yield). Note that this compound is unstable so only partial characterization is given. ${ }^{1} \mathrm{H}$ NMR (400 MHz, $\left.\mathrm{CDCl}_{3}\right): \delta 9.72(\mathrm{~s}, 1 \mathrm{H}), 9.39(\mathrm{~s}, 1 \mathrm{H}), 7.38(\mathrm{~m}, 3 \mathrm{H}), 7.28(\mathrm{~m}, 2 \mathrm{H}), 6.55$ 
(t, $J=7.2 \mathrm{~Hz}, 1 \mathrm{H}), 2.58(\mathrm{q}, J=7.2 \mathrm{~Hz}, 2 \mathrm{H}), 2.50(\mathrm{t}, J=7.6 \mathrm{~Hz}, 2 \mathrm{H}), 2.42(\mathrm{t}, J=7.6 \mathrm{~Hz}$, 2H), 2.32 (t, $J=8.0 \mathrm{~Hz}, 2 \mathrm{H}$ ), 1.83 (quint, $J=7.2 \mathrm{~Hz}, 2 \mathrm{H}$ ), 1.70 (quint, $J=8.0 \mathrm{~Hz}, 2 \mathrm{H}$ ); ${ }^{13} \mathrm{C} \mathrm{NMR}\left(125 \mathrm{MHz}, \mathrm{CDCl}_{3}\right): \delta \quad 202.3,195.2,155.0,143.6,131.7,128.5,128.0,123.8$, 89.0, 81.9, 43.7, 28.2, 27.8, 23.5, 21.5, 21.3, 19.3; IR $\left(\mathrm{CDCl}_{3}\right.$, film, $\left.\mathrm{cm}^{-1}\right): 2930(\mathrm{~m})$, 2865(w), 2718(w), 1723(s), 1682(vs), 1640(m), 1598(w), 1490(m), 1442(m), 1290(w), 1144(w), 1069(m) 758(s), 693(s); HRMS (EI) $m / z$ calcd for $\mathrm{C}_{18} \mathrm{H}_{20} \mathrm{O}_{2}\left[\mathrm{M}^{+}\right]$267.1385, found 267.1383.

Synthesis of Tricyclic Spiro Compound, 25.
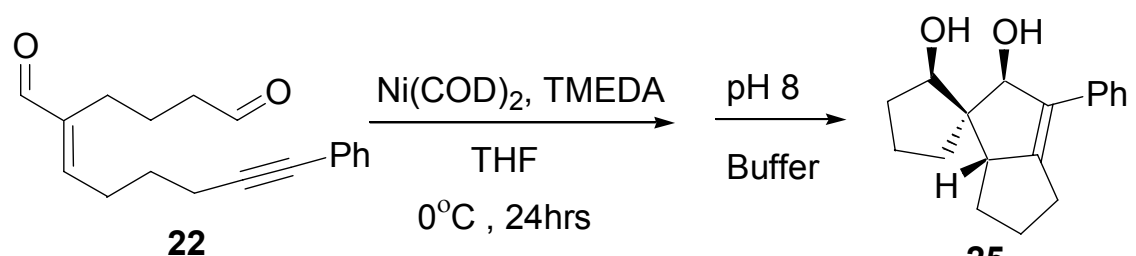

25

\section{5, Spirocompound:}

A $25 \mathrm{~mL}$ round bottom flask was charged with $\mathrm{Ni}(\mathrm{COD})_{2}(25 \mathrm{mg}, 0.091 \mathrm{mmol}, 1$ eq. $)$ and $4 \mathrm{~mL}$ THF followed by the addition of TMEDA (15 $\mu \mathrm{L}, 0.099 \mathrm{mmol}, 1.1 \mathrm{eq}$.) and chilled to $0^{\circ} \mathrm{C}$. The yellow mixture was stirred for 10 minutes before the dropwise addition of 22 (24 mg, $0.089 \mathrm{mmol}$, 1eq.) as a solution in $7 \mathrm{~mL} \mathrm{CH}_{2} \mathrm{Cl}_{2}$ via a syringe over about 1 minute. Within 2 minutes, the reaction mixture had become an orange color and after 25 minutes the reaction had turned a deep red color. The reaction was quenched after 24 hours with pH 8 buffer. Column chromatography (hexanes: ethyl acetate, 2:1) afforded a yellow, oily solid (mp 91 $\left.{ }^{\circ} \mathrm{C}\right), 25$ (12 mg, $\left.0.043 \mathrm{mmol}, 49 \%\right) .{ }^{1} \mathrm{H}$ NMR (400 $\mathrm{MHz}$, $\left.\mathrm{CDCl}_{3}\right): \delta 7.45(\mathrm{~d}, J=7.2 \mathrm{~Hz}, 2 \mathrm{H}), 7.36(\mathrm{t}, J=7.2 \mathrm{~Hz}, 2 \mathrm{H}), 7.22(\mathrm{t}, J=7.2 \mathrm{~Hz}, 1 \mathrm{H})$, $4.98(\mathrm{~s}, 1 \mathrm{H}), 4.36(\mathrm{t}, J=8.8 \mathrm{~Hz}, 1 \mathrm{H}), 3.65(\mathrm{~m}, 1 \mathrm{H}) 2.89(\mathrm{~s}, 1 \mathrm{H}), 2.61(\mathrm{dd}, J=18.6,9.6$ 
$\mathrm{Hz}, 1 \mathrm{H}), 2.36-2.45$ (m, 1H), 2.04-2.22 (m, 3H), 1.85 (br s, $1 \mathrm{H}), 1.79$ (dt, $J=11.6,6.4 \mathrm{~Hz}$, 1H), $1.53-1.74(\mathrm{~m}, 4 \mathrm{H}), 1.32-1.55(\mathrm{~m}, 2 \mathrm{H}) ;{ }^{13} \mathrm{C} \mathrm{NMR}\left(100 \mathrm{MHz}, \mathrm{CDCl}_{3}, \mathrm{DEPT}\right) \delta$ $156.9\left(\mathrm{C}_{\text {quat. }}\right), 135.2\left(\mathrm{C}_{\text {quat. }}\right), 130.1\left(\mathrm{C}_{\text {quat. }}\right), 128.9(\mathrm{CH}), 126.7(\mathrm{CH}), 91.4(\mathrm{CH}), 76.5(\mathrm{CH})$, $56.8\left(\mathrm{C}_{\text {quat. }}\right), 52.5(\mathrm{CH}), 31.5\left(\mathrm{CH}_{2}\right), 30.9\left(\mathrm{CH}_{2}\right), 29.8\left(\mathrm{CH}_{2}\right), 27.0\left(\mathrm{CH}_{2}\right), 26.6\left(\mathrm{CH}_{2}\right), 19.1$ $\left(\mathrm{CH}_{2}\right)$; Gradient HMQC (400MHz, $\mathrm{CDCl}_{3}$, selected peaks, $\left.{ }^{1} \mathrm{H} \leftrightarrow{ }^{13} \mathrm{C}\right): \delta 7.45 \leftrightarrow 128.9$, $7.36 \leftrightarrow 126.7, \quad 7.22 \leftrightarrow 126.7, \quad 4.98 \leftrightarrow 91.4, \quad 4.36 \leftrightarrow 76.5, \quad 3.65 \leftrightarrow 52.5, \quad 1.65 \leftrightarrow 31.5$, $2.06 \leftrightarrow 31.5,1.79 \leftrightarrow 27.0,1.62 \leftrightarrow 19.1$; Gradient DQFCOSY (400MHz, $\mathrm{CDCl}_{3}$, selected peaks, $\left.\quad{ }^{1} \mathrm{H} \leftrightarrow{ }^{1} \mathrm{H}\right): \quad \delta \quad 4.36 \leftrightarrow 2.10 ; 1.65, \quad 3.65 \leftrightarrow 2.61 ; 1.79 ; 1.36, \quad 2.61 \leftrightarrow 2.40 ; 3.65$, $2.40 \leftrightarrow 2.61 ; 2.10 ; 2.06,2.18 \leftrightarrow 1.36 ; 2.40,1.79 \leftrightarrow 1.36,1.66 \leftrightarrow 1.36 ; 2.12 ;$

Gradient $\mathrm{NOE}\left(400 \mathrm{MHz}, \mathrm{CDCl}_{3}\right.$, selected peaks, Irradiate $\left.\leftrightarrow \mathrm{NOE}[\%]\right): \delta$ $7.45 \leftrightarrow 4.98[3.8], \quad 4.98 \leftrightarrow 7.45[8.5]$ and $4.36[3.2], \quad 4.36 \leftrightarrow 4.98[0.82]$ and 2.11[4.1], $3.65 \leftrightarrow 1.81$ and $1.56[9.8], 2.61 \leftrightarrow 7.45[5.3]$; IR (film, $\mathrm{cm}^{-1}$ ): 3399(br w), 2923(vs), 1733(m), 1650(w), 1599(w),1489(m), 1458(m), 1378(m), 1258(m), 1091(s), 1072(m), 836(m), 755(s), 690(s); LRMS (EI) $m / z$ calcd for $\mathrm{C}_{18} \mathrm{H}_{22} \mathrm{O}_{2}[\mathrm{M}+\mathrm{Na}]^{+}$293.03., found 293.04. Stereochemical assignments were confirmed by single crystal X-ray analysis.
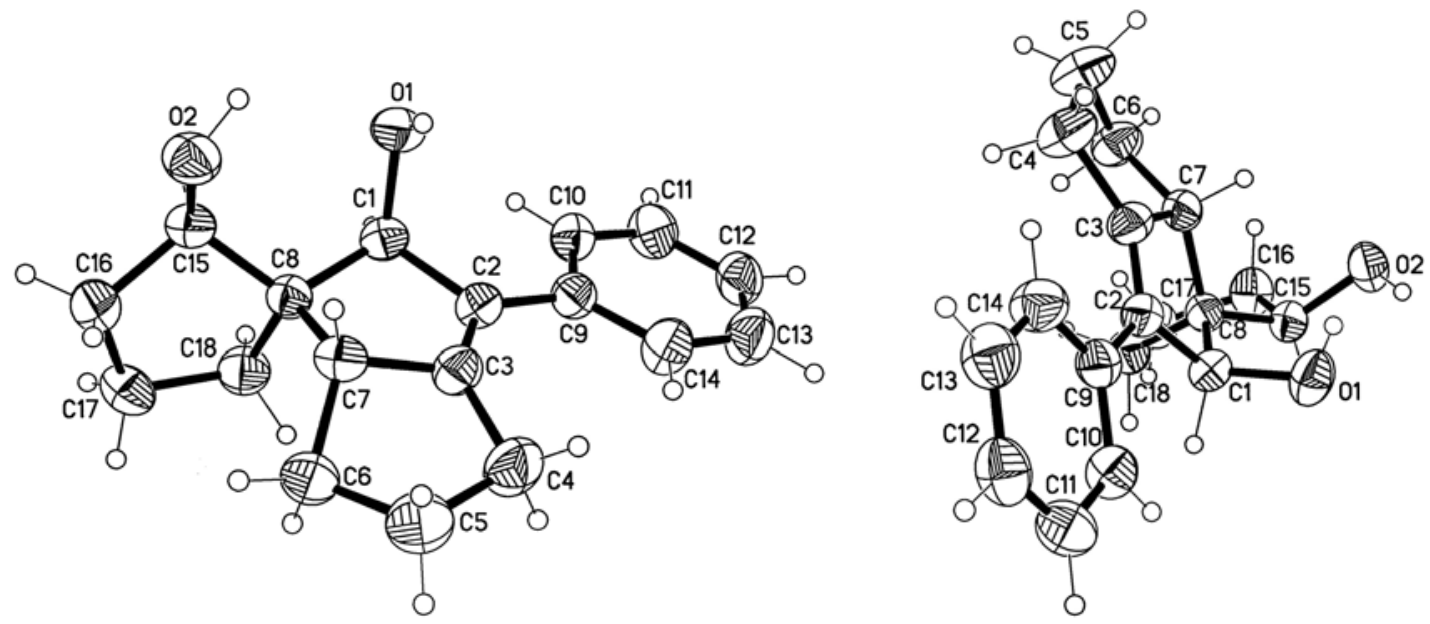

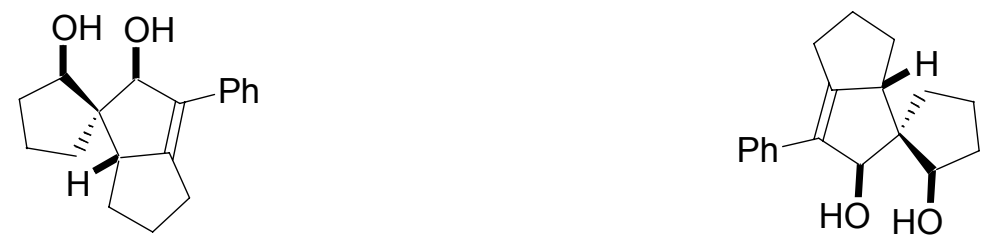

Figure 2: ORTEP perspective views of 25 showing the labeling system. Thermal ellipsoids are drawn at a 30\% probability level.

\section{Experimental Scheme D: Towards Tricyclic Compound 29 (Scheme 7)}
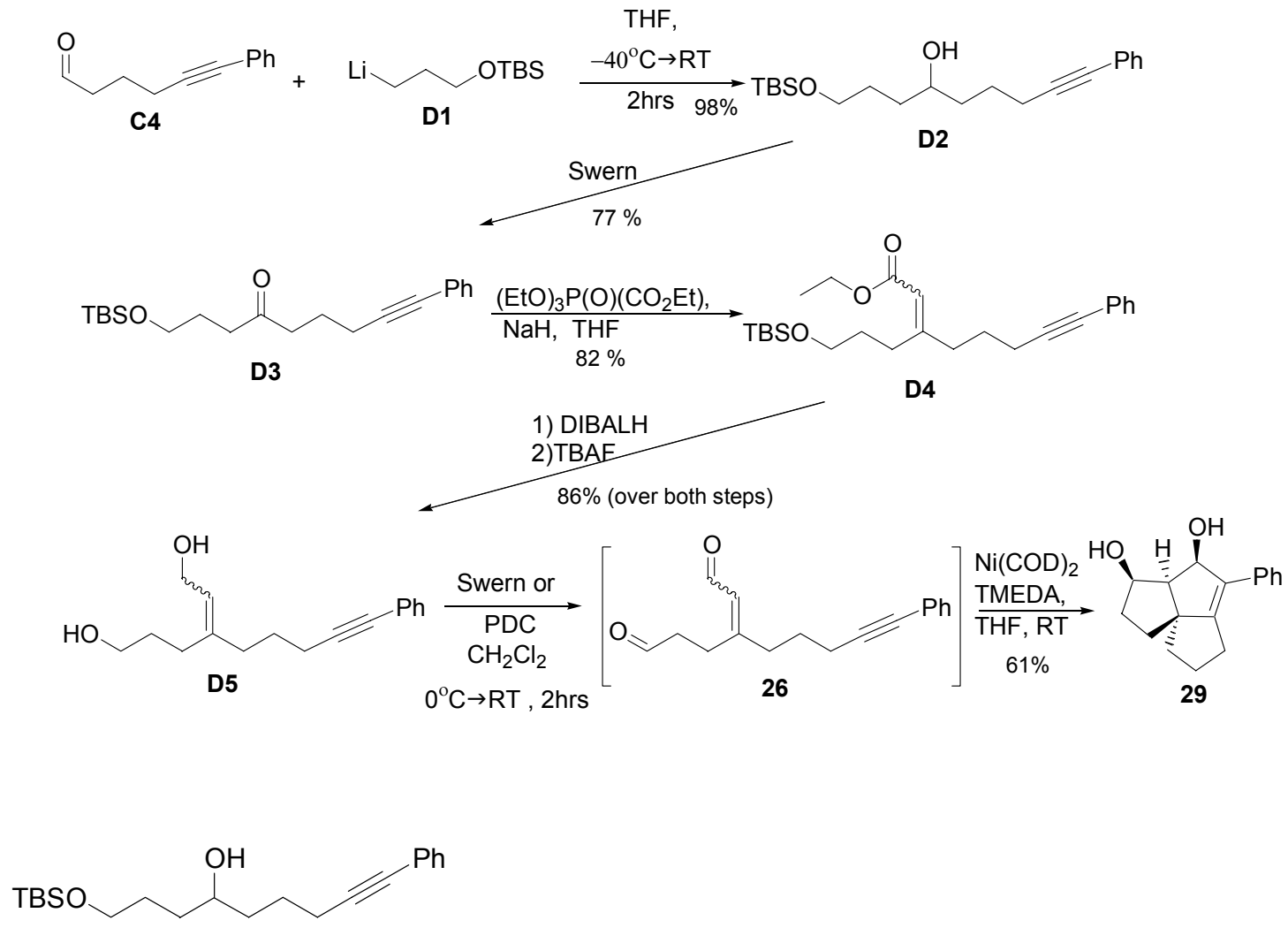

D2

\section{D2, 1-(tert-Butyl-dimethyl-silanyloxy)-9-phenyl-non-8-yn-4-ol:}

A $5 \mathrm{~mL} \mathrm{Et}_{2} \mathrm{O}$ solution of $\mathbf{C 4}(1.37 \mathrm{~g}, 7.89 \mathrm{mmol})$ was added dropwise to a $-40^{\circ} \mathrm{C}$ solution of D1 (10.5 mL, 0.75 M solution in hexanes, $7.88 \mathrm{mmol}, 1$ eq., FMC Corporation, Lithium division) in $20 \mathrm{~mL}$ diethyl ether. After $50 \mathrm{~min}$, the yellow reaction mixture was warmed to $0^{\circ} \mathrm{C}$. The resulting light orange reaction mixture was stirred at this temperature for $30 \mathrm{~min}$ followed by warming to $\mathrm{rt}$ with continued stirring for another 30 
min. The reaction mixture was quenched with saturated aqueous ammonium chloride, and the organic material was extracted with ether and dried over anhydrous magnesium sulfate. Column chromatography (4:1 Hex/EtOAc) afforded D2 (2.69g, $7.76 \mathrm{mmol}$, 98\%) as a colorless oil. ${ }^{1} \mathrm{H}$ NMR (500 MHz, $\left.\mathrm{CDCl}_{3}\right): \delta 7.38(\mathrm{~m}, 2 \mathrm{H}), 7.25-7.29(\mathrm{~m}, 3 \mathrm{H})$, $3.67(\mathrm{~m}, 3 \mathrm{H}), 2.72(\mathrm{br} \mathrm{s}, 1 \mathrm{H}), 2.44(\mathrm{t}, J=7.0 \mathrm{~Hz}, 2 \mathrm{H}), 1.74-1.81(\mathrm{~m}, 1 \mathrm{H}), 1.57-1.72(\mathrm{~m}$, $6 \mathrm{H}), 1.44-1.52(\mathrm{~m}, 1 \mathrm{H}), 0.90(\mathrm{~s}, 9 \mathrm{H}), 0.07(\mathrm{~s}, 6 \mathrm{H}) ;{ }^{13} \mathrm{C} \mathrm{NMR}\left(125 \mathrm{MHz}, \mathrm{CDCl}_{3}\right) \delta$ $131.7,128.4,127.7,124.2,90.4,81.0,71.3,63.8,36.8,35.2,29.4,26.1,25.3,19.7,18.5$, -5.2; IR (film, $\mathrm{cm}^{-1}$ ) 3374 (br, w), 2928(m), 2856(m), 1599(w), 1490(m), 1462(m), 1442(m), 1254(m), 1092(m), 1069(m), 1005(m), $912(\mathrm{w}), 833(\mathrm{~s}), 776(\mathrm{~m}), 754(\mathrm{vs})$, 690(vs); HRMS (EI) $m / z$ calcd for $\mathrm{C}_{21} \mathrm{H}_{34} \mathrm{O}_{2} \mathrm{Si}\left[\mathrm{M}^{+}\right] 346.2328$, found $346.2328\left(\mathrm{M}^{+}\right)$.

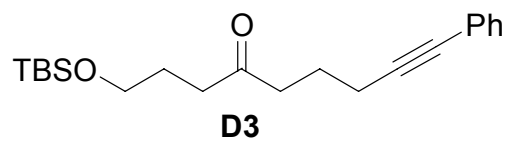

\section{D3, 1-(tert-Butyl-dimethyl-silanyloxy)-9-phenyl-non-8-yn-4-one:}

A solution of dimethylsulfoxide $(25 \mu \mathrm{L}, 0.346 \mathrm{mmol})$ was added dropwise via syringe to a $-78^{\circ} \mathrm{C}$ solution of oxalyl chloride $(15 \mu \mathrm{L}, 0.173 \mathrm{mmol})$ in $1 \mathrm{~mL}$ of $\mathrm{CH}_{2} \mathrm{Cl}_{2}$. The reaction mixture was stirred at this temperature for $20 \mathrm{~min}$ before a solution of 1-(tbutyldimethylsilanyloxy)-9-phenyl-non-8-yn-4-ol (30mg, $0.087 \mathrm{mmol}$ ) in $1 \mathrm{~mL} \mathrm{CH}_{2} \mathrm{Cl}_{2}$ was introduced. After stirring $40 \mathrm{~min}$ at $-78^{\circ} \mathrm{C}$, triethylamine $(72 \mu \mathrm{L}, 0.519 \mathrm{mmol})$ was then added and the mixture was allowed to warm to rt. The reaction mixture was quenched by pouring into a saturated solution of sodium bicarbonate. The organic material was extracted with $3 \mathrm{x}$ with ethyl acetate. The solvent was removed in vacuo and column chromatography of the resulting crude mixture (10:1 Hex/EtOAc) afforded (23 
$\mathrm{mg}, 0.067 \mathrm{mmol}, 77 \%$ ) of the product $\mathbf{D 3}$ as a colorless oil. ${ }^{1} \mathrm{H}$ NMR (500 MHz, $\mathrm{CDCl}_{3}$ ): $\delta$ 7.37-7.39 (m, 2H), 7.25-7.29 (m, 3H), $3.60(\mathrm{t}, J=6.0 \mathrm{~Hz}, 2 \mathrm{H}), 2.62(\mathrm{t}, J=7.0 \mathrm{~Hz}, 2 \mathrm{H})$, $2.52(\mathrm{t}, J=7.5 \mathrm{~Hz}, 2 \mathrm{H}), 2.45$ (t, $J=7.0 \mathrm{~Hz}, 2 \mathrm{H}), 1.87$ (quint, $J=7.0 \mathrm{~Hz}, 2 \mathrm{H}), 1.79$ (m, 2H), $0.88(\mathrm{~s}, 9 \mathrm{H}), 0.03(\mathrm{~s}, 6 \mathrm{H}) ;{ }^{13} \mathrm{C} \mathrm{NMR}\left(125 \mathrm{MHz}, \mathrm{CDCl}_{3}\right) \delta 210.7,131.8,128.4$, $127.9,124.0,89.4,81.5,62.4,41.6,39.5,27.0,26.122 .8,19.0,18.5,-5.1$; IR (film, $\mathrm{cm}^{-1}$ ): 3060 (w), 2955 (s), 2932 (s), 2856 (m), 1713 (vs), 1598(w), 1489(s), 1463(m), 1442(m), $1409(\mathrm{~m}), 1371(\mathrm{~m}), 1255(\mathrm{~m}), 1097(\mathrm{~m}), 1028(\mathrm{w}), 1005(\mathrm{w}), 914(\mathrm{w}), 835(\mathrm{~s}), 776(\mathrm{~m})$, 756(vs), 692(vs); HRMS (EI) $\mathrm{m} / z$ calcd for $\mathrm{C}_{21} \mathrm{H}_{32} \mathrm{O}_{2} \mathrm{Si} 344.2172$ [M $\mathrm{M}^{+}$, found 287.1465 $[\mathrm{M}-(t \text {-butyl })]^{+}$.

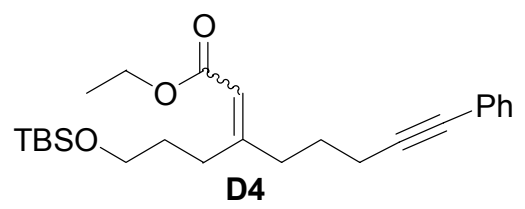

D4, 3-[3-(tert-Butyl-dimethyl-silanyloxy)-propyl]-8-phenyl-oct-2-en-7-ynoic acid ethyl ester:

Sodium hydride (149 $\mathrm{mg}$ of $60 \%$ dispersion in oil, $3.77 \mathrm{mmol}, 2.0$ eq.) was washed with $2 \times 5 \mathrm{~mL}$ portions of hexanes (hexane layer subsequently discarded) and the resulting powder suspended in $15 \mathrm{~mL}$ THF. The suspension was cooled to $-78^{\circ} \mathrm{C}$ prior to the dropwise addition of triethyl phosphonoacetate $(0.935 \mathrm{~mL}, 4.71 \mathrm{mmol}, 2.0$ eq. $)$ in $2 \mathrm{~mL}$ THF and warmed to $\mathrm{rt}$ and stirred at this temperature for $30 \mathrm{~min}$. The reaction mixture was cooled to $-78^{\circ} \mathrm{C}$ and a solution of $\mathbf{D 3}(650 \mathrm{mg}, 1.88 \mathrm{mmol})$ in $2 \mathrm{~mL}$ THF was added dropwise over $10 \mathrm{~min}$. The mixture was warmed to $\mathrm{rt}$ and then heated to $65^{\circ} \mathrm{C}$ overnight. After TLC analysis indicated the reaction was complete $\left(4: 1 \mathrm{Hex} / \mathrm{EtOAc}, \mathrm{R}_{\mathrm{f}}=0.68\right.$, visualized as with anisaldehyde stain), the reaction was quenched with saturated 
ammonium chloride. The organic layer was extracted with ether and dried over magnesium sulfate. Column chromatography $(10: 1 \mathrm{Hex} / \mathrm{EtOAc})$ of the crude material gave a colorless oil containing an inseparable 1:1 E:Z mixture of D4 (642 mg, 1.55 mmol, 82\%). $\quad{ }^{1} \mathrm{H}$ NMR (500 MHz, $\left.\mathrm{CDCl}_{3}\right): \delta 7.38(\mathrm{~m}, 2 \mathrm{H}), 7.27(\mathrm{~m}, 3 \mathrm{H}), 5.69(\mathrm{~s}, 1 \mathrm{H})$, $4.14(\mathrm{q}, J=6.4 \mathrm{~Hz}, 2 \mathrm{H}), 3.66(\mathrm{t}, J=6.4 \mathrm{~Hz}, 1 \mathrm{H}), 3.62(\mathrm{t}, J=5.6 \mathrm{~Hz}, 1 \mathrm{H}), 2.76(\mathrm{t}, J=8.0$ $\mathrm{Hz}, 1 \mathrm{H}), 2.66(\mathrm{t}, J=7.2 \mathrm{~Hz}, 1 \mathrm{H}), 2.45(\mathrm{dt}, J=13.6,7.2 \mathrm{~Hz}, 2 \mathrm{H}), 2.34(\mathrm{t}, J=8.0 \mathrm{~Hz}, 1 \mathrm{H})$, $2.25(\mathrm{t}, J=8.0 \mathrm{~Hz}, 1 \mathrm{H}), 1.79$ (quint, $J=7.6 \mathrm{~Hz}, 2 \mathrm{H}), 1.66-1.73(\mathrm{~m}, 2 \mathrm{H}), 1.27(\mathrm{t}, J=7.6$ $\mathrm{Hz}, 3 \mathrm{H}), 0.89$ (s, 9H), 0.04 (s, 6H); ${ }^{13} \mathrm{C} \mathrm{NMR}\left(125 \mathrm{MHz}, \mathrm{CDCl}_{3}\right): \delta 166.65,166.59$, $163.4,163.3,131.8,128.45,128.40,127.88,127.75,124.19,123.99,116.2,90.08,89.50$, $81.54,81.20,63.39,62.56,59.8,37.63,35.00,32.02,31.77,30.9,28.84,28.03,26.83$, 26.2, 19.91, 19.25, 18.54, 14.6, -5.04, -5.07; IR (film, $\mathrm{cm}^{-1}$ ): 2952 (m), 2929 (m), 2856 (m), 2253 (w), 1713 (vs), 1642 (s), 1599(w), 1490(m), 1462(m), 1442(m), 1379 (m), 1255(s), 1226 (s), 1160 (vs), 1099(vs), 1038(s), 1006(m), 965 (m), 910 (s), 834(vs), 775 (s), 756(vs), 732 (vs), 691(s); HRMS (EI) $\mathrm{m} / z$ calcd for $\mathrm{C}_{25} \mathrm{H}_{38} \mathrm{O}_{3} \mathrm{Si} \quad\left[\mathrm{M}^{+}\right]$414.2590, found 414.2591.

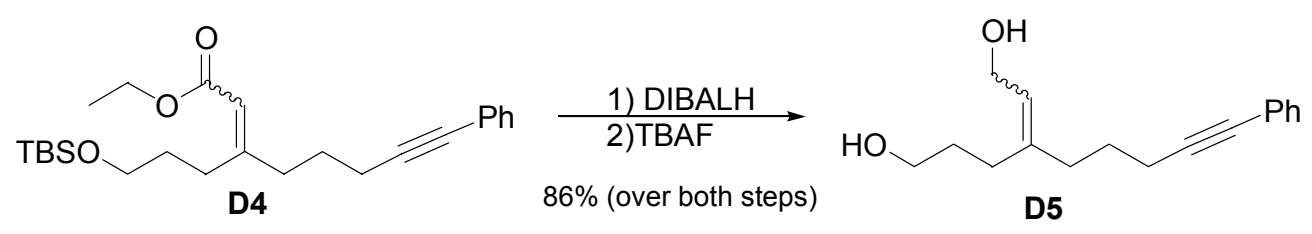

\section{D5, 3-(5-Phenyl-pent-4-ynyl)-hex-2-ene-1,6-diol:}

A solution of $\mathbf{D} 4$ (363 mg, $0.876 \mathrm{mmol}, 1$ eq.) in $10 \mathrm{~mL}$ of $\mathrm{CH}_{2} \mathrm{Cl}_{2}$ was cooled to $-40^{\circ} \mathrm{C}$ and stirred $20 \mathrm{~min}$ before the dropwise addition of DIBALH (2.70 mL, $1.0 \mathrm{M}$ in hexanes, $2.70 \mathrm{mmol}, 3.1 \mathrm{eq}$.$) . Three hours later, TLC analysis (4:1 Hex:EtOAc; stained with$ vanillin) indicated the reaction was complete. At $-40^{\circ} \mathrm{C}$ the reaction mixture was 
quenched with $1 \mathrm{~mL}$ methanol and allowed to warm to rt. The reaction mixture was poured into a 1:1 mixture of ethyl acetate:saturated potassium sodium tartrate and stirred 15 min before extracting $3 \mathrm{x}$ with ether. The organic layer was dried over magnesium sulfate and the solvent removed in vacuo to give a yellow residue. This residue was taken up in $20 \mathrm{~mL}$ THF and cooled to $0^{\circ} \mathrm{C}$. TBAF $(2.0 \mathrm{~mL}, 0.90 \mathrm{M}$ in THF, $1.8 \mathrm{mmol}$, 2.0 eq.) was added dropwise; the mixture was stirred at $0^{\circ} \mathrm{C}$ for $1 \mathrm{~h}$ resulting in the reaction mixture turning from yellow to orange. The reaction was quenched with $5 \mathrm{~mL}$ 1.0 N HCl. The product was extracted with ether and the organic layer dried over anhydrous magnesium sulfate. Column chromatography $\left(5 \% \mathrm{MeOH}\right.$ in $\left.\mathrm{CH}_{2} \mathrm{Cl}_{2}\right)$ afforded a yellow oil of D5 (194.3 $\mathrm{mg}, 0.752 \mathrm{mmol}, 86 \%$ over both steps) as an inseparable $\sim 1: 1$ mixture of $E$ and $Z$ isomers. $\left.\quad{ }^{1} \mathrm{H} \mathrm{NMR} \mathrm{(500} \mathrm{MHz,} \mathrm{CDCl}_{3}\right): \delta$ 7.36-7.40 (m, 2H), 7.25$7.29(\mathrm{~m}, 3 \mathrm{H}), 5.59(\mathrm{t}, J=7.5 \mathrm{~Hz}, 0.5 \mathrm{H}), 5.48(\mathrm{t}, J=7.0 \mathrm{~Hz}, 0.5 \mathrm{H}), 4.19(\mathrm{~d}, J=7.0 \mathrm{~Hz}$, $1 \mathrm{H}), 4.11(\mathrm{~d}, J=7.5 \mathrm{~Hz}, 1 \mathrm{H}), 3.62(\mathrm{t}, J=6.5 \mathrm{~Hz}, 1 \mathrm{H}), 3.56(\mathrm{t}, J=5.5 \mathrm{~Hz}, 1 \mathrm{H}), 3.02(\mathrm{br}$ $\mathrm{s}, 1 \mathrm{H}), 2.39$ (q, $J=6.5 \mathrm{~Hz}, 2 \mathrm{H}), 2.24-2.28(\mathrm{~m}, 2 \mathrm{H}), 2.18(\mathrm{t}, J=7.5 \mathrm{~Hz}, 1 \mathrm{H}), 2.0-2.3(\mathrm{br} \mathrm{s}$, $1 \mathrm{H}), 2.12(\mathrm{t}, J=7.5 \mathrm{~Hz}, 1 \mathrm{H}), 1.65-1.75(\mathrm{~m}, 4 \mathrm{H}) ;{ }^{13} \mathrm{C} \mathrm{NMR}\left(125 \mathrm{MHz}, \mathrm{CDCl}_{3}\right): \delta 143.3$, $142.1,131.7,128.49,128.44,127.89,127.81,125.3,125.0,124.12,123.99,90.08,89.89$, $81.44,81.27,62.5,61.0,59.1,58.4,35.2,32.9,30.9,30.3,29.3,27.6,27.0,25.6,19.2$ IR (film, $\left.\mathrm{cm}^{-1}\right)$ : 3338(br, s), 2938(s), 2868(m), 2253(w), 1663(w), 1598(w), 1490(m), 1442(m), 1379(m), 1058(s), 1004(s), 914(w), 834(vs), 756(vs), 692(vs); HRMS (EI) m/z calcd for $\mathrm{C}_{17} \mathrm{H}_{22} \mathrm{O}_{2}[\mathrm{M}-\mathrm{H}]^{+}$258.1620, found 257.1540. 


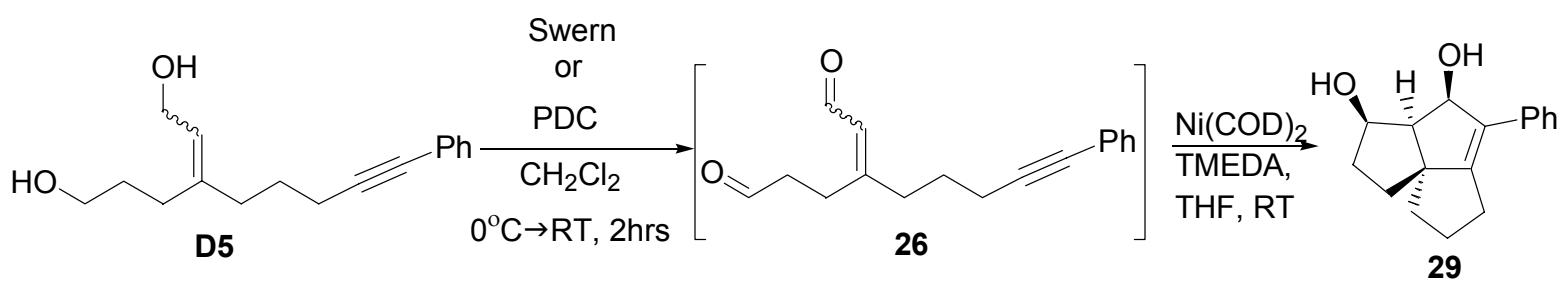

\section{9, 5-Phenyl-1,2,3,3a,4,6,7,8-octahydro-cyclopenta[c]pentalene-3,4-diol:}

Method a) D5 $\rightarrow \mathbf{2 6}$, isolate, then $\mathbf{2 6} \rightarrow \mathbf{2 9}$

A $25 \mathrm{~mL}$ round bottom flask was charged with $\mathbf{D 5}(41.4 \mathrm{mg}, 0.160 \mathrm{mmol}, 1$ eq.) and 5 $\mathrm{mL}$ of methylene chloride; the flask was cooled to $0{ }^{\circ} \mathrm{C}$. PDC (142 $\mathrm{mg}, 0.377 \mathrm{mmol}, 2.3$ eq) was added in two equal lots with a 6 minute interval. The mixture turned from yellow to red as the reaction proceeded and warmed to room temperature where it remained stirring under nitrogen for 2 hours. TLC analysis confirmed that the reaction was complete (blue spot with vanillin stain). The reaction mixture was filtered through Celite and dried over anhydrous magnesium sulfate. Column chromatography (2:1 Hex:EtOAc) of the residue gave $\mathbf{2 6},(40.2 \mathrm{mg}, 0.158 \mathrm{mmol}, 99 \%)$ as a yellow oil. Due to the instability of 26, only partial characterization was possible. ${ }^{1} \mathrm{H}$ NMR $\left(500 \mathrm{MHz}, \mathrm{CDCl}_{3}\right)$ : $\delta 10.08(\mathrm{~d}, 1 \mathrm{H}, J=7.5 \mathrm{~Hz}), 9.81(\mathrm{~s}), 7.38(\mathrm{~m}, 2 \mathrm{H}), 7.29(\mathrm{~m}, 3 \mathrm{H}), 5.86(\mathrm{~d}, 1 \mathrm{H}, J=8.0 \mathrm{~Hz})$, $2.80(\mathrm{t}, 2 \mathrm{H}, J=7.5 \mathrm{~Hz}), 2.72(\mathrm{~d}, 2 \mathrm{H}, J=7.0 \mathrm{~Hz}), 2.59(\mathrm{t}, 2 \mathrm{H}, J=7.2 \mathrm{~Hz}), 2.50(\mathrm{t}, 2 \mathrm{H}, J=6.5$ $\mathrm{Hz}$ ), 1.87 (quint, 2H, $J=6.5 \mathrm{~Hz}$ ); ${ }^{13} \mathrm{C}$ NMR (125 MHz, $\left.\mathrm{CDCl}_{3}\right): \delta 200.1,190.8,164.4$, 131.7, 128.5, 128.1, 123.6, 88.6, 82.2, 41.4, 30.7, 29.7, 28.3, 26.5, 19.3; IR $\left(\mathrm{CDCl}_{3}\right.$, film, $\left.\mathrm{cm}^{-1}\right): 1722(\mathrm{~s}), 1669(\mathrm{vs}), 1490(\mathrm{~m})$.

$26 \rightarrow 29$

To a stirring solution of $\mathrm{Ni}(\mathrm{COD})_{2}(10 \mathrm{mg}, 0.036 \mathrm{mmol}, 1$ eq. $)$ and TMEDA $(10 \mu \mathrm{L}$, $0.066 \mathrm{mmol}, 1.8$ eq.) in $5 \mathrm{~mL} \mathrm{THF}$ at $0^{\circ} \mathrm{C}, 26(9.2 \mathrm{mg}, 0.036 \mathrm{mmol})$ was added dropwise as a solution in $2 \mathrm{~mL}$ of THF. Within $2 \mathrm{~min}$, the yellow solution had turned color to 
orange-brown, and then while warming to rt the reaction mixture became red. After 3 hours, the reaction was complete by TLC then quenched with a $\mathrm{pH} 8$ buffer solution. The organic layer was extracted with ether and dried over magnesium sulfate. Column chromatography (2:1 hexanes: ethyl acetate) gave the clean separation of a light yellow oil, 29 (4.9 mg, $0.019 \mathrm{mmol}, 53 \%)$. This material was spectroscopically identical (details follow) to the material obtained by the preferred method below.

\section{Method b) D5 $\rightarrow[\mathbf{2 6}$ (in situ) $] \rightarrow \mathbf{2 9}$}

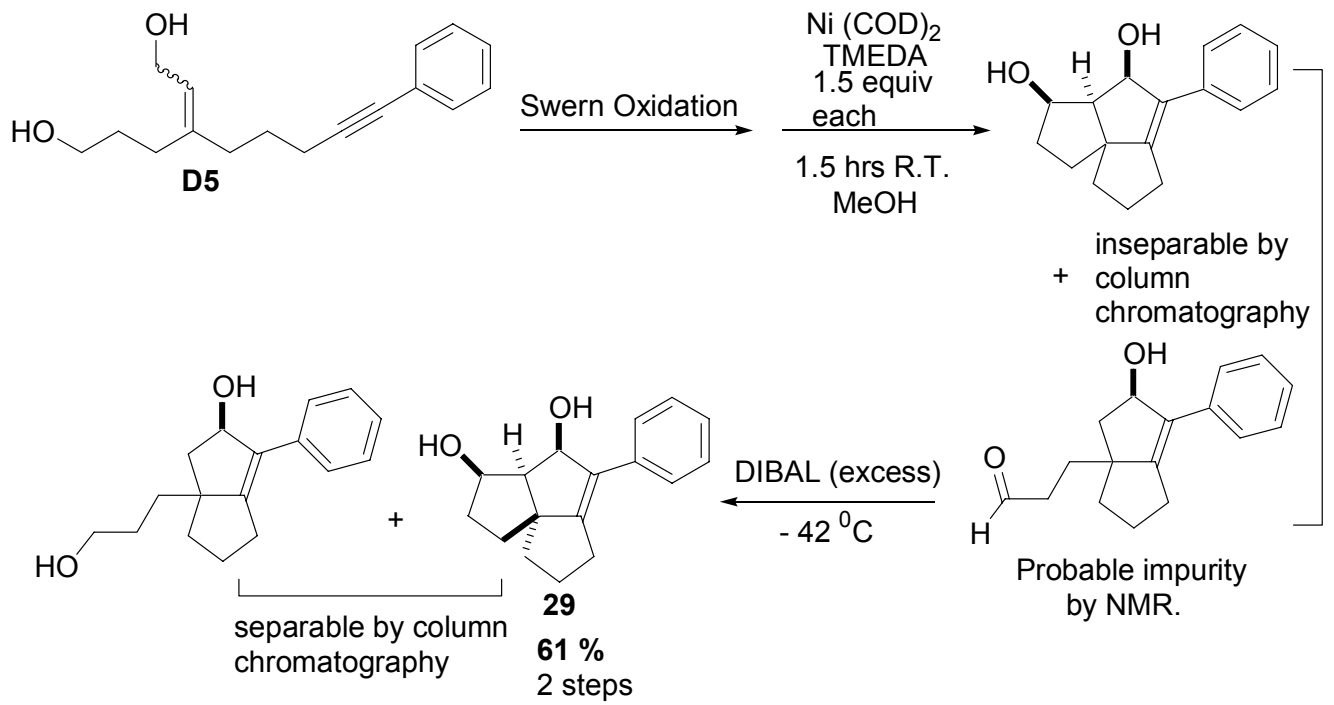

Swern oxidation of the mixture of cis and trans isomers of D5 (40 mg, $0.155 \mathrm{mmol})$ followed by workup and solvent removal gave the crude mixture containing enyne-dial 26. This was then introduced in the nickel cyclization using the general procedure. The crude mixture, $\mathrm{Ni}(\mathrm{COD})_{2}(64 \mathrm{mg}, 0.232 \mathrm{mmol}, 1.5 \mathrm{eq}$.$) and tmeda (35 \mu \mathrm{L}, 0.232 \mathrm{mmol})$ were stirred for $1.5 \mathrm{~h}$ at $\mathrm{rt}$, followed by addition of anhydrous methanol $(2 \mathrm{~mL})$. Silica gel column chromatography $(3: 1 \mathrm{Hex} / \mathrm{EtOAc})$ gave the product $\mathbf{2 9}$ with an impurity which most likely was the monocyclized compound by NMR analysis. Since the mixture could not be separated by column chromatography, the mixture was treated with excess 
DIBAL at $-40^{\circ} \mathrm{C}$. Subsequent workup and chromatographic purification (10:1 Hex/EtOAc) afforded 29 (24mg, $0.093 \mathrm{mmol}, 61 \%$ over 2 steps $)$ as a white crystalline solid (mp 130-131 $\left.{ }^{\circ} \mathrm{C}\right) .{ }^{1} \mathrm{H}$ NMR (400 MHz, $\left.\mathrm{CDCl}_{3}\right) \delta$ 7.35-7.46 (m, 4H), 7.23-7.26 (m, $1 \mathrm{H}), 5.81(\mathrm{~d}, J=8.0 \mathrm{~Hz}, 1 \mathrm{H}), 4.39(\mathrm{q}, J=8.5 \mathrm{~Hz}, 1 \mathrm{H}), 4.05(\mathrm{~s}, 1 \mathrm{H}), 2.56-2.67(\mathrm{~m}, 3 \mathrm{H})$, $2.32(\mathrm{dt}, J=17.0,8.5 \mathrm{~Hz}, 1 \mathrm{H}), 1.94-2.12(\mathrm{~m}, 4 \mathrm{H}), 1.86-1.91(\mathrm{~m}, 1 \mathrm{H}), 1.68(\mathrm{dd}, J=11.5$, $7.0 \mathrm{~Hz}, 1 \mathrm{H}), 1.31-1.44(\mathrm{~m}, 2 \mathrm{H}) ;{ }^{13} \mathrm{C} \mathrm{NMR}\left(100 \mathrm{MHz}, \mathrm{CDCl}_{3}\right) \delta 156.6,134.4,132.4$, $128.9,127.5,127.2,84.4,77.1,65.4,50.0,38.6,35.1,33.2,26.6,24.3$. Irradiation of the methine signal at $\delta 5.81$ (allylic $\mathrm{C}-\mathrm{H}-\mathrm{OH}$ ) resulted in $9.3 \% \mathrm{NOE}$ enhancement to the methine signal at 2.56-2.67 (tertiary C-프). IR (film, $\mathrm{cm}^{-1}$ ) 3388 (br m), 1497 (m), 1444 (m), 1262 (m), 1090 (s), 1065 (s), 1034 (s), 1013 (s), 909 (m), 801 (m), 769 (s), 693 (vs); HRMS (EI) $m / z$ calcd for $\mathrm{C}_{17} \mathrm{H}_{20} \mathrm{O}_{2} 256.1463\left[\mathrm{M}^{+}\right]$, found 256.1461. Stereochemical assignments were confirmed by single crystal X-ray analysis.
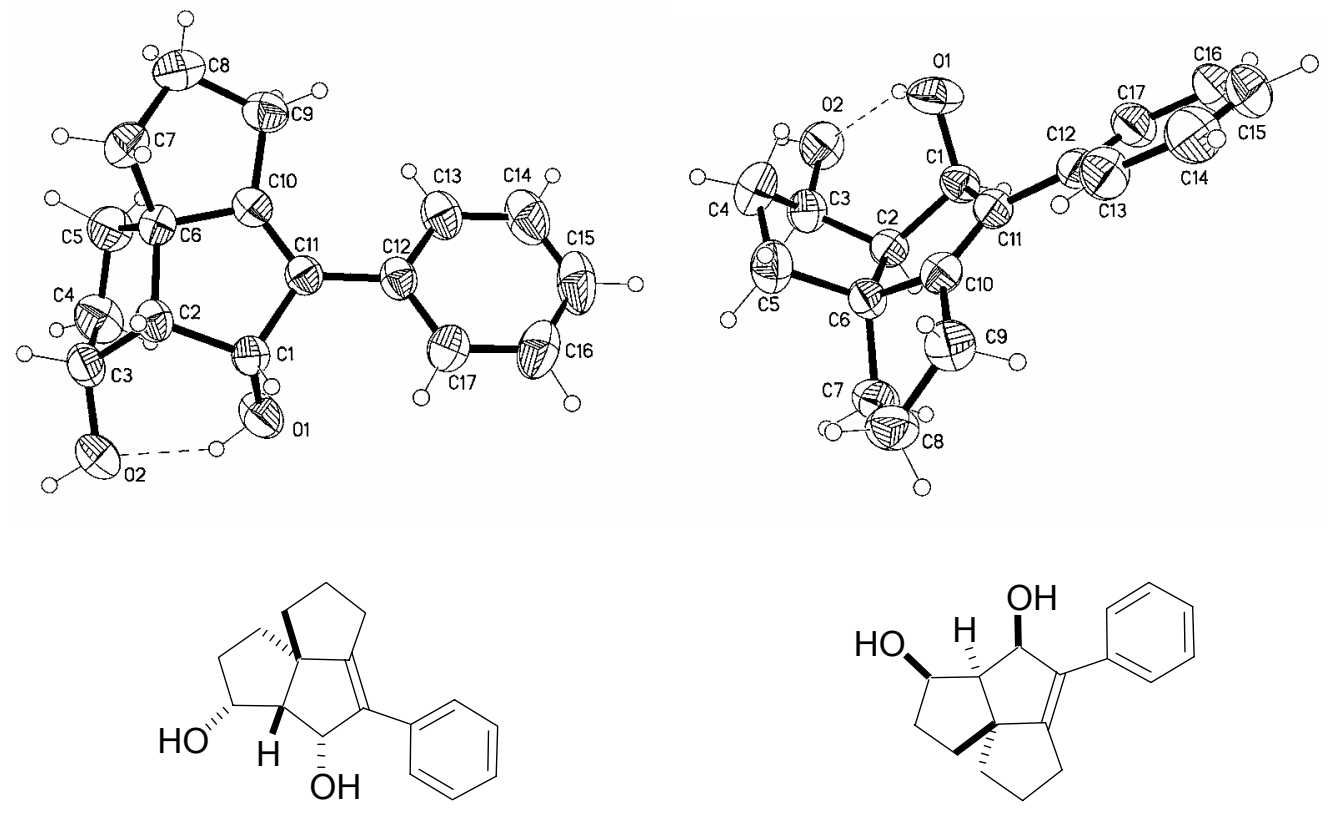

Figure 3: ORTEP perspective views of 29 showing the labeling system. Thermal ellipsoids are drawn at a $30 \%$ probability level. 
Experimental procedures for compounds leading up to and including Scheme 8 are represented as that shown for Table 2, Entry 3. 
${ }^{1}$ H NMR Spectra 


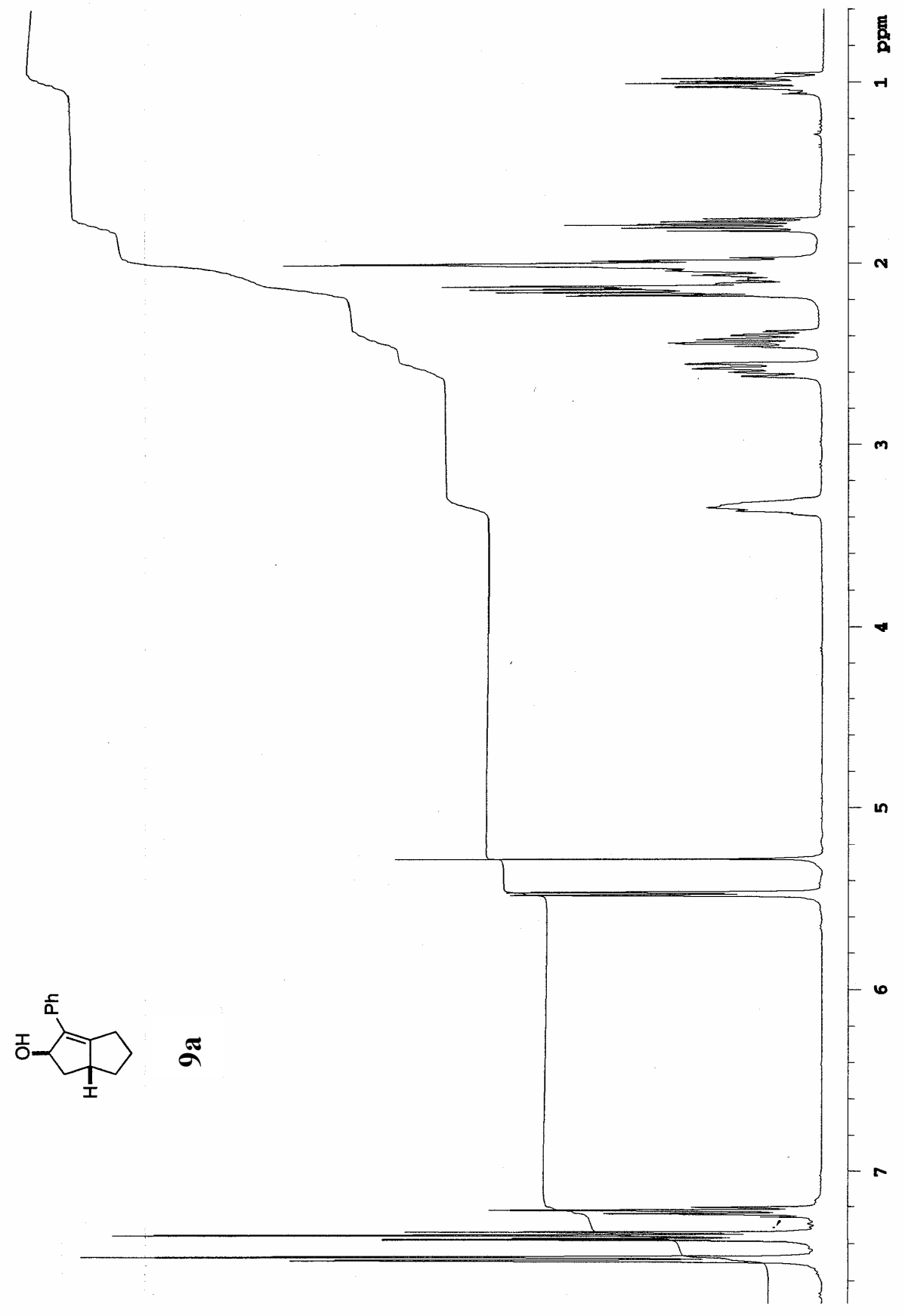




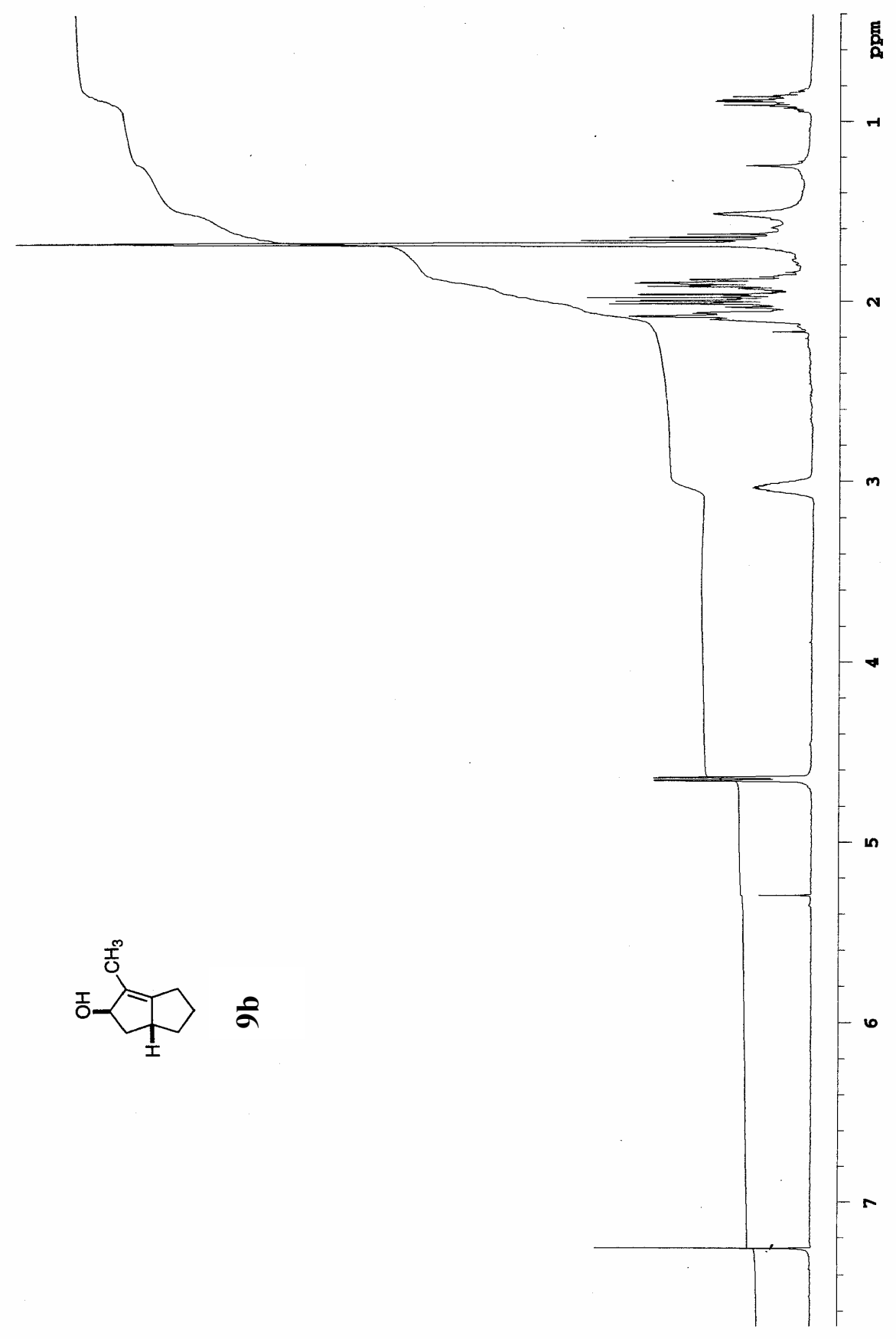




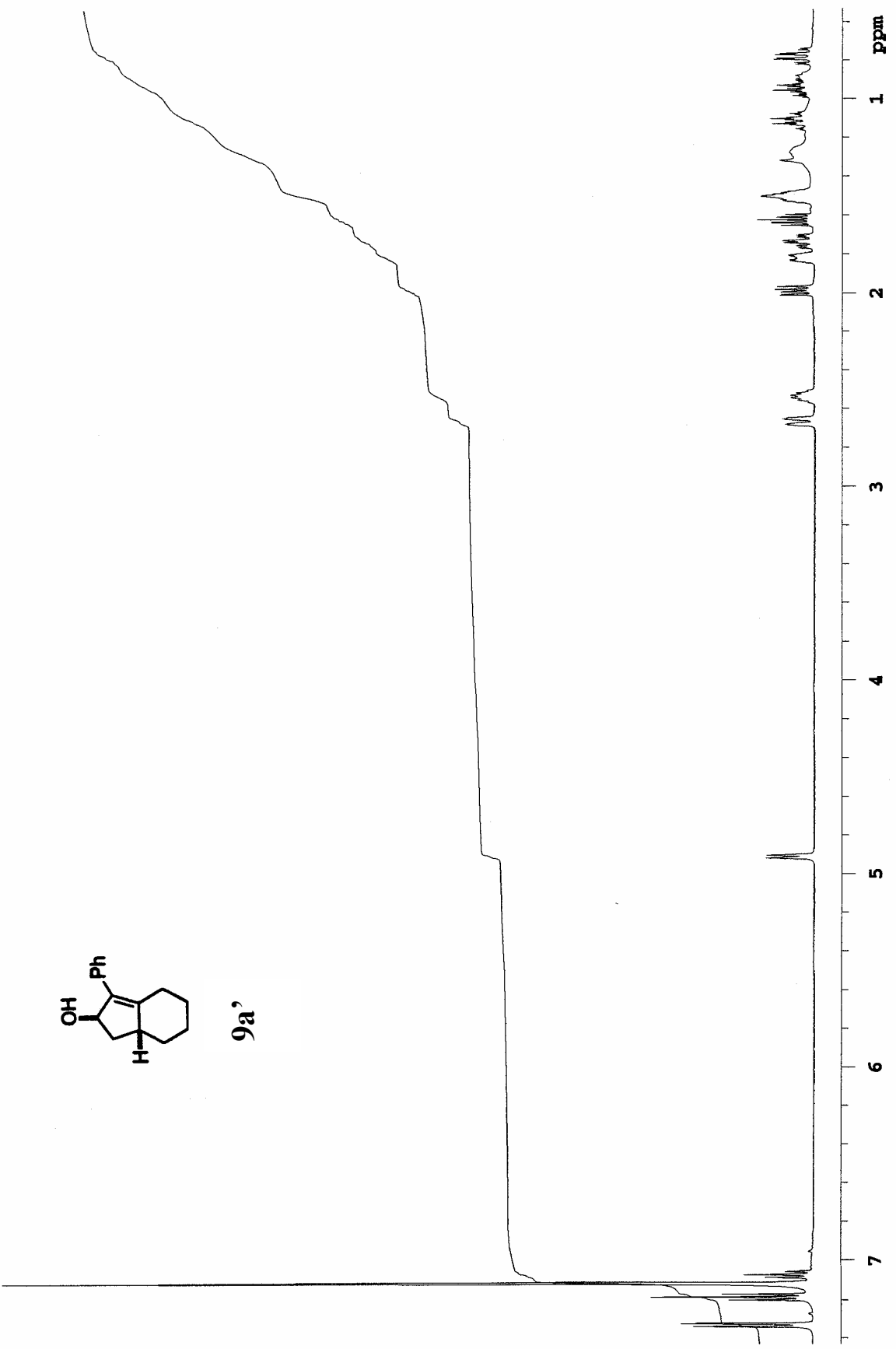




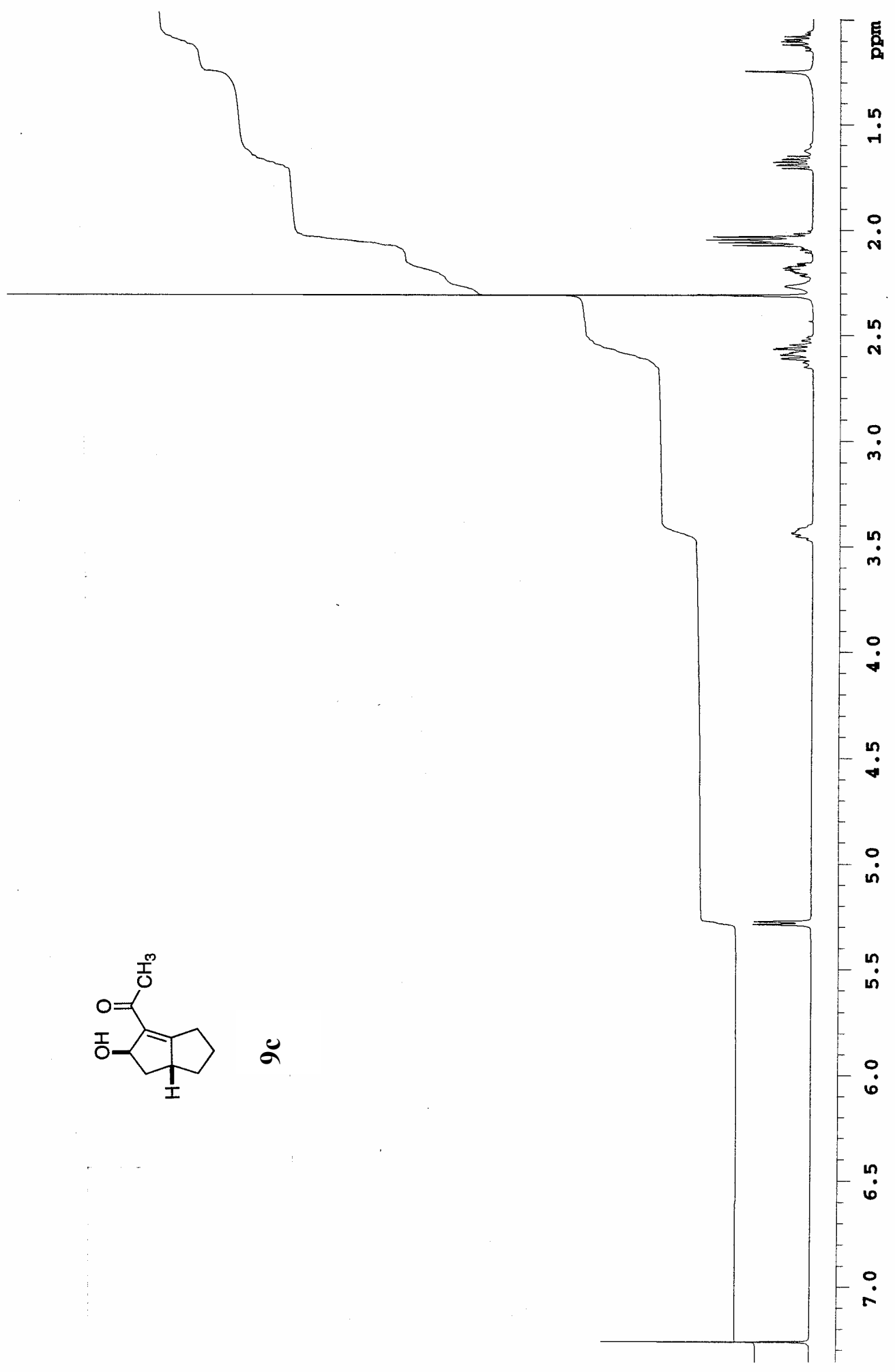


S48

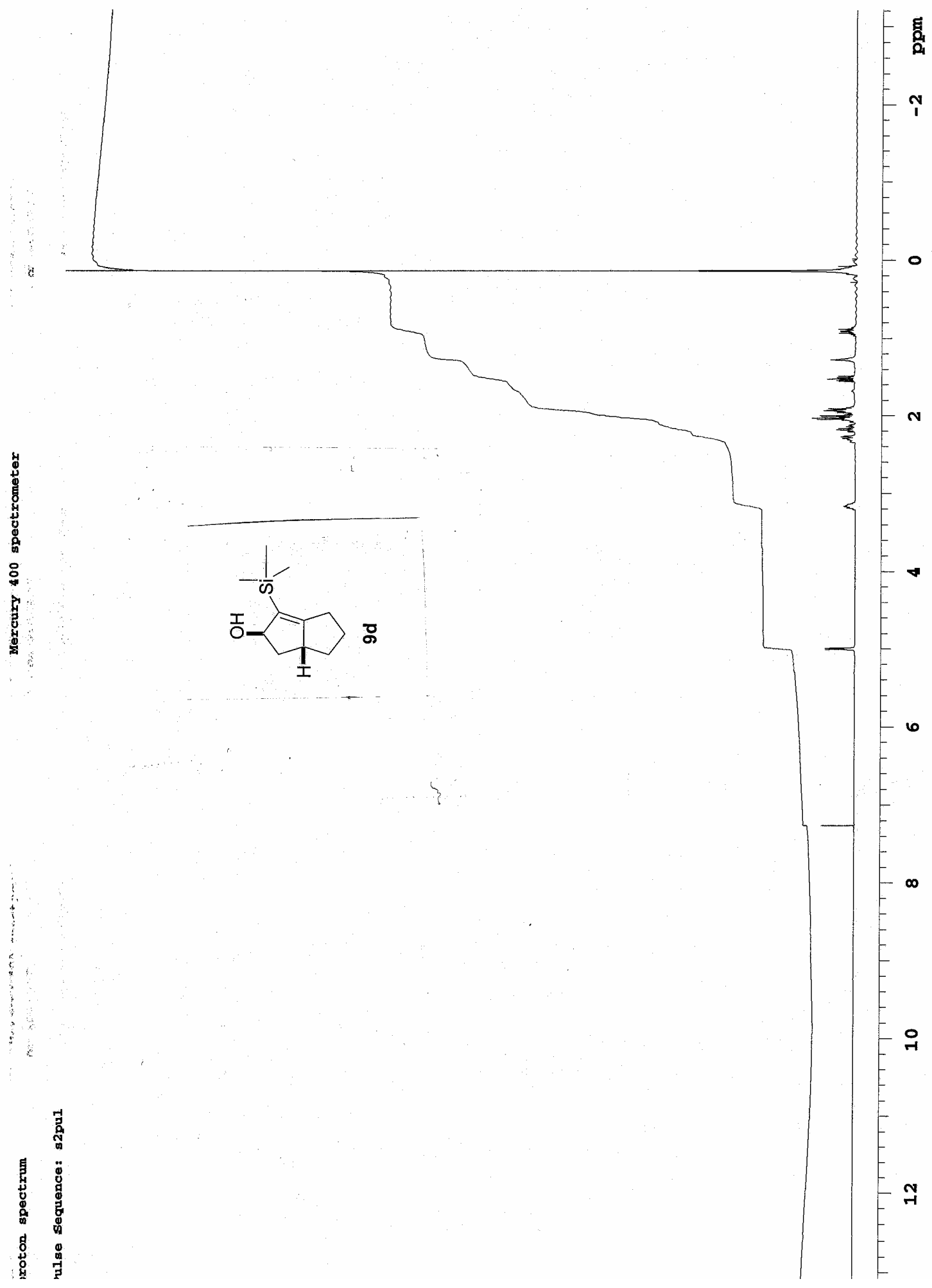




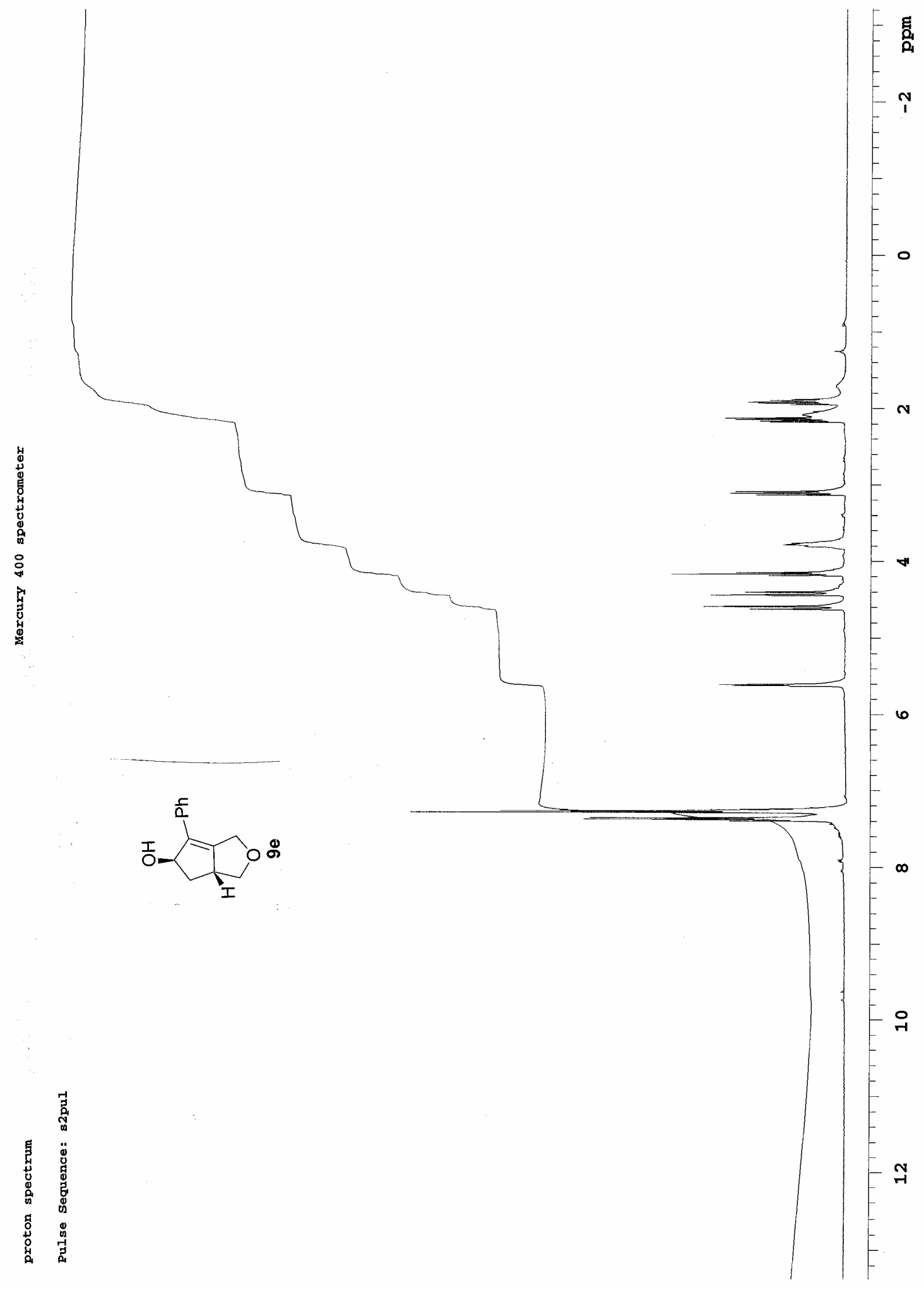




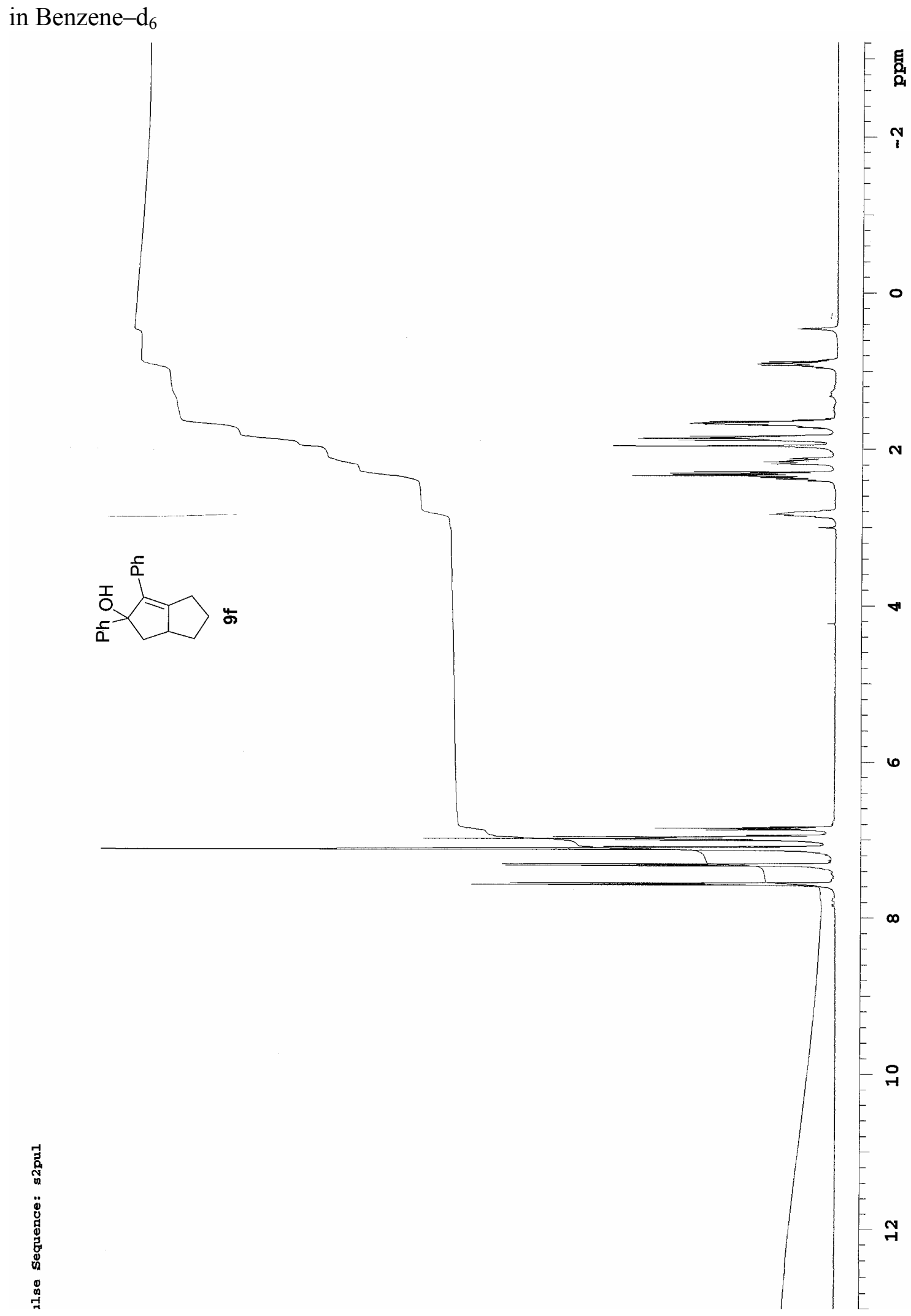




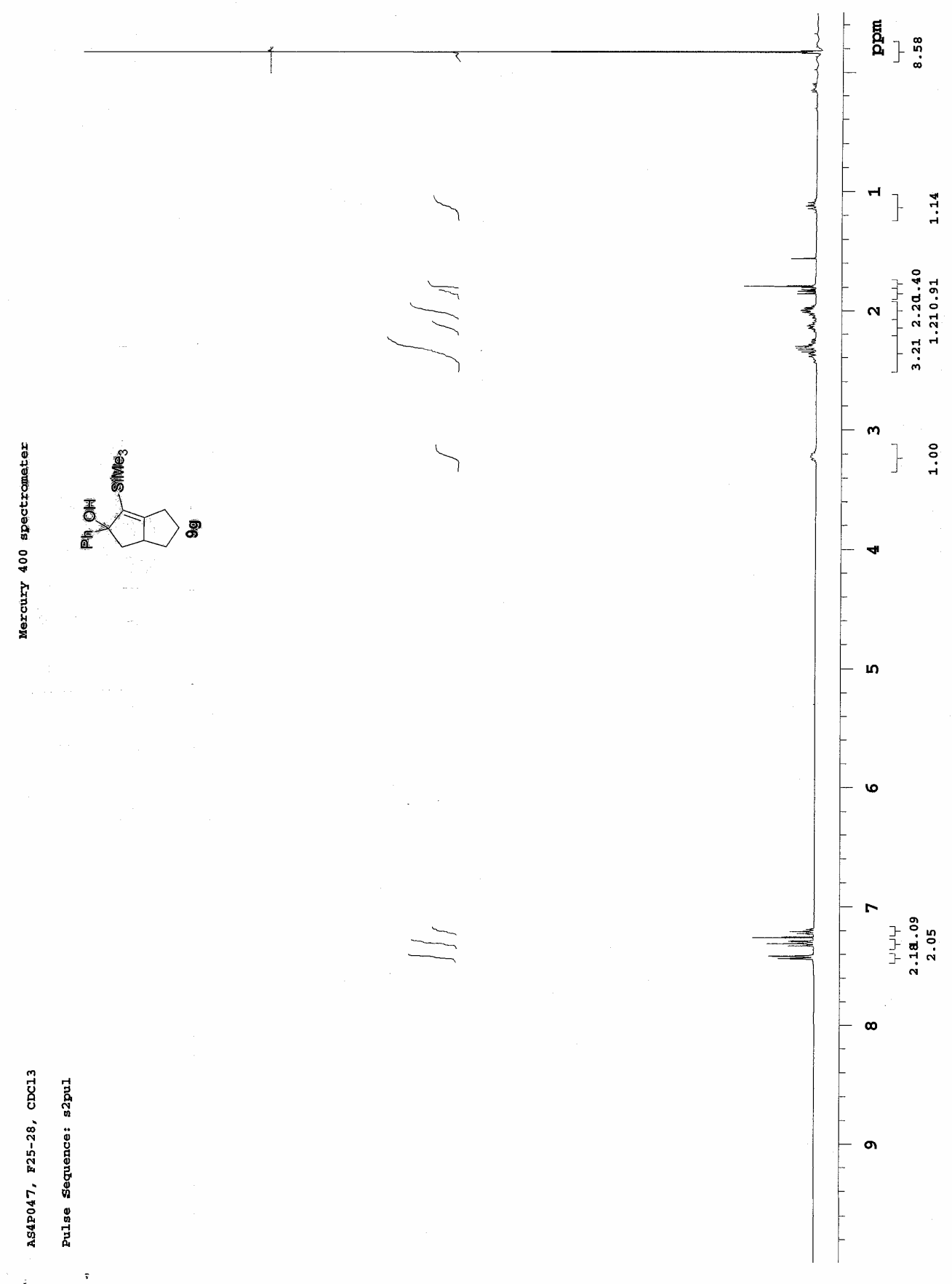




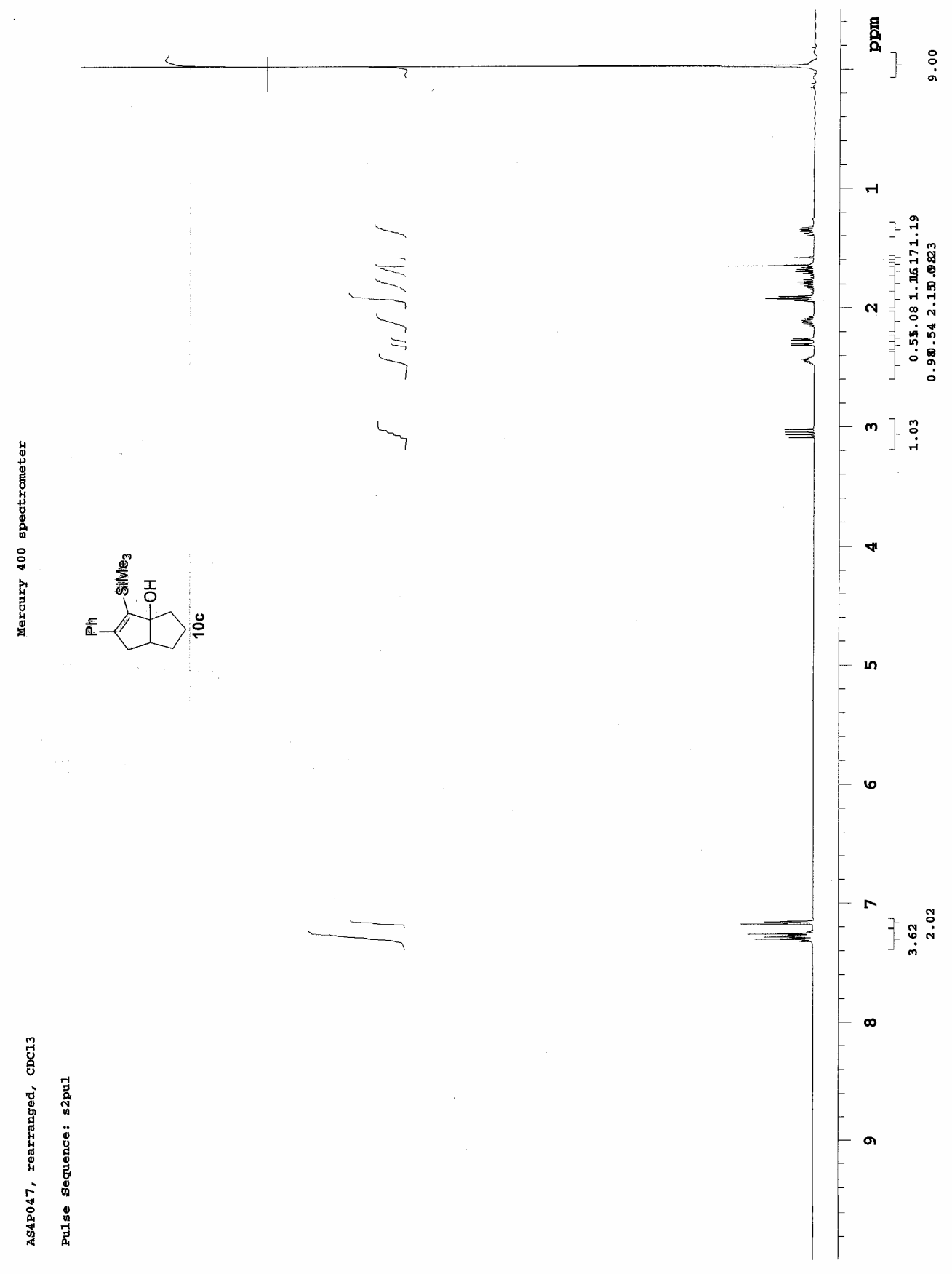




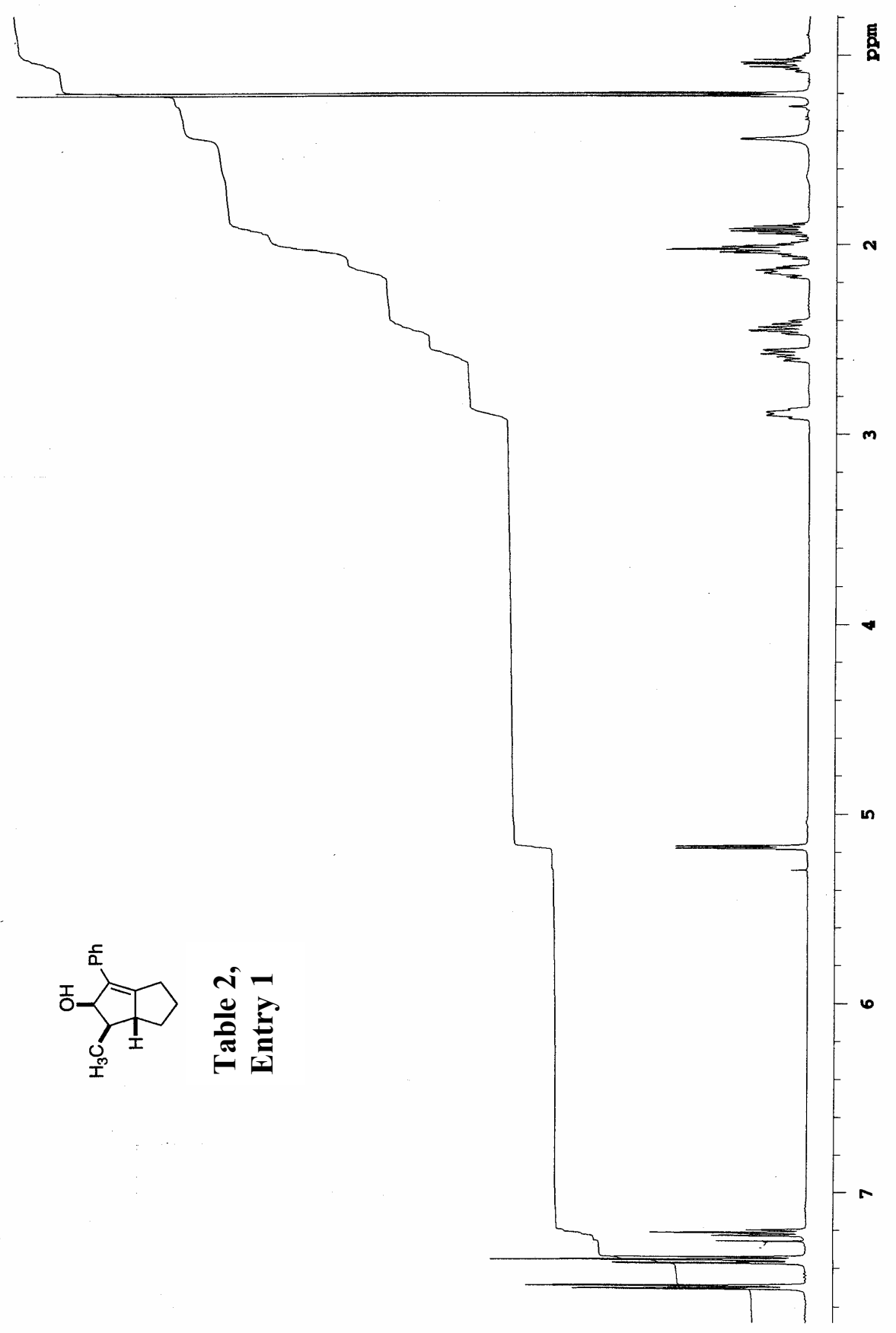




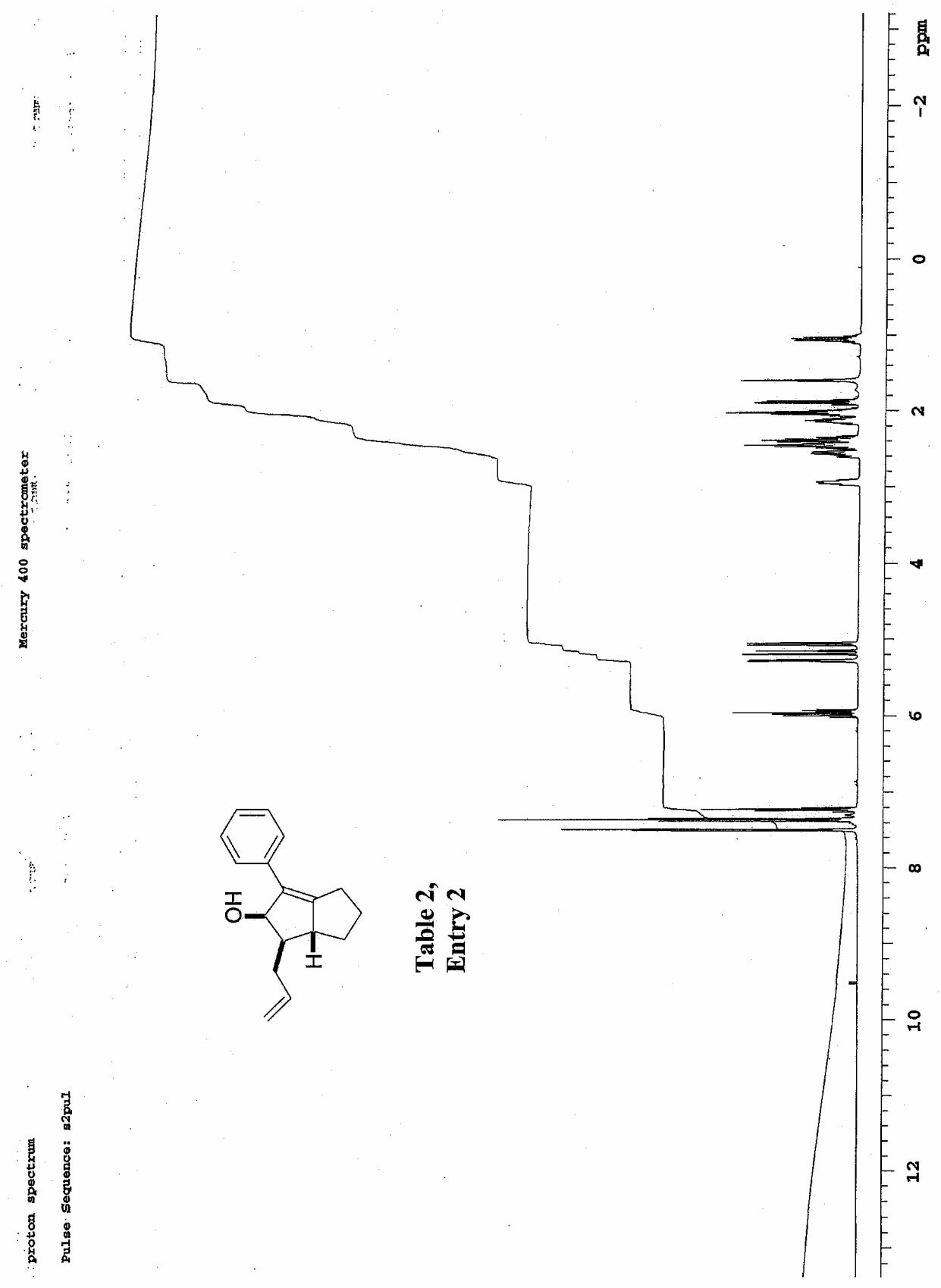




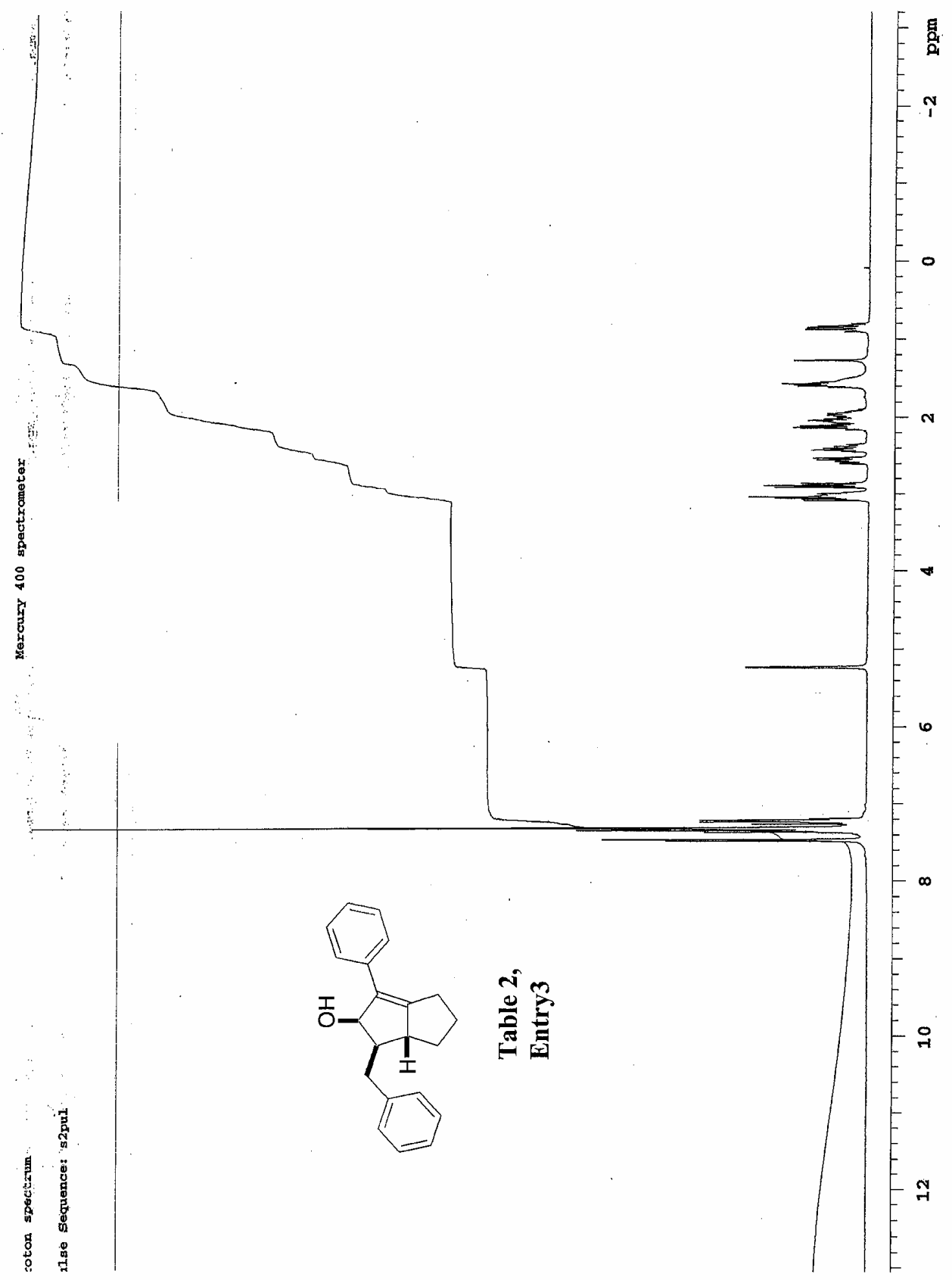




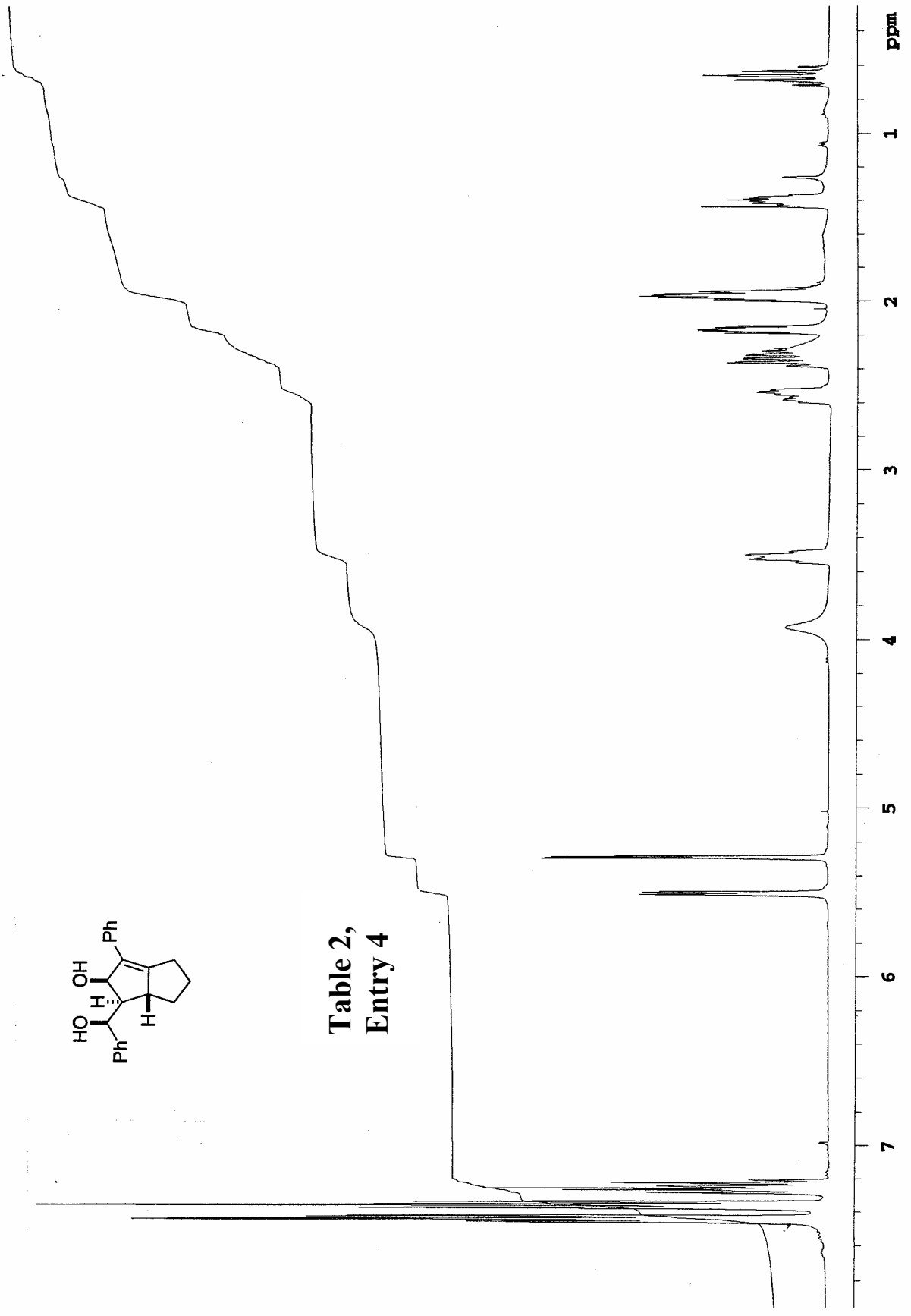




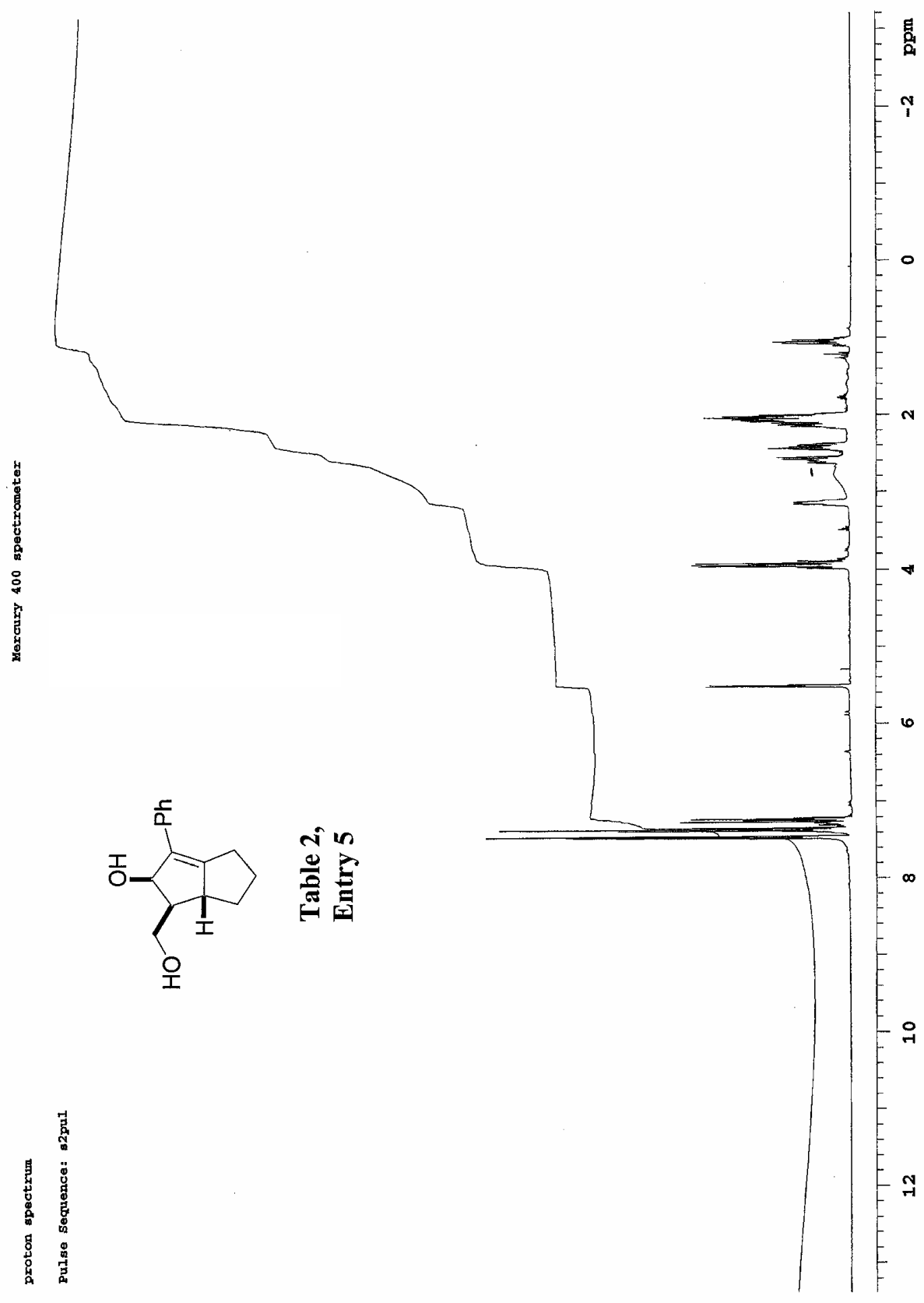




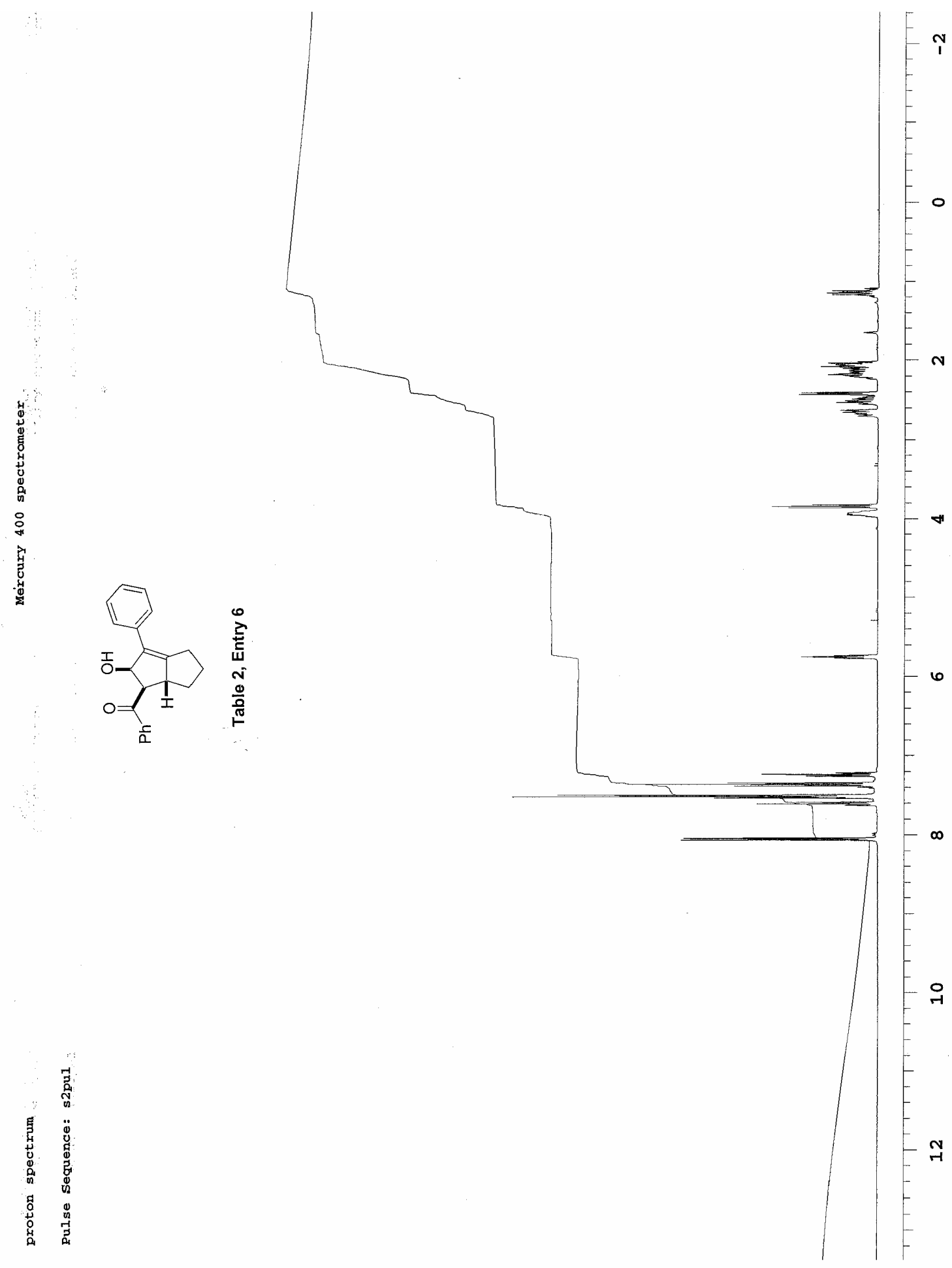




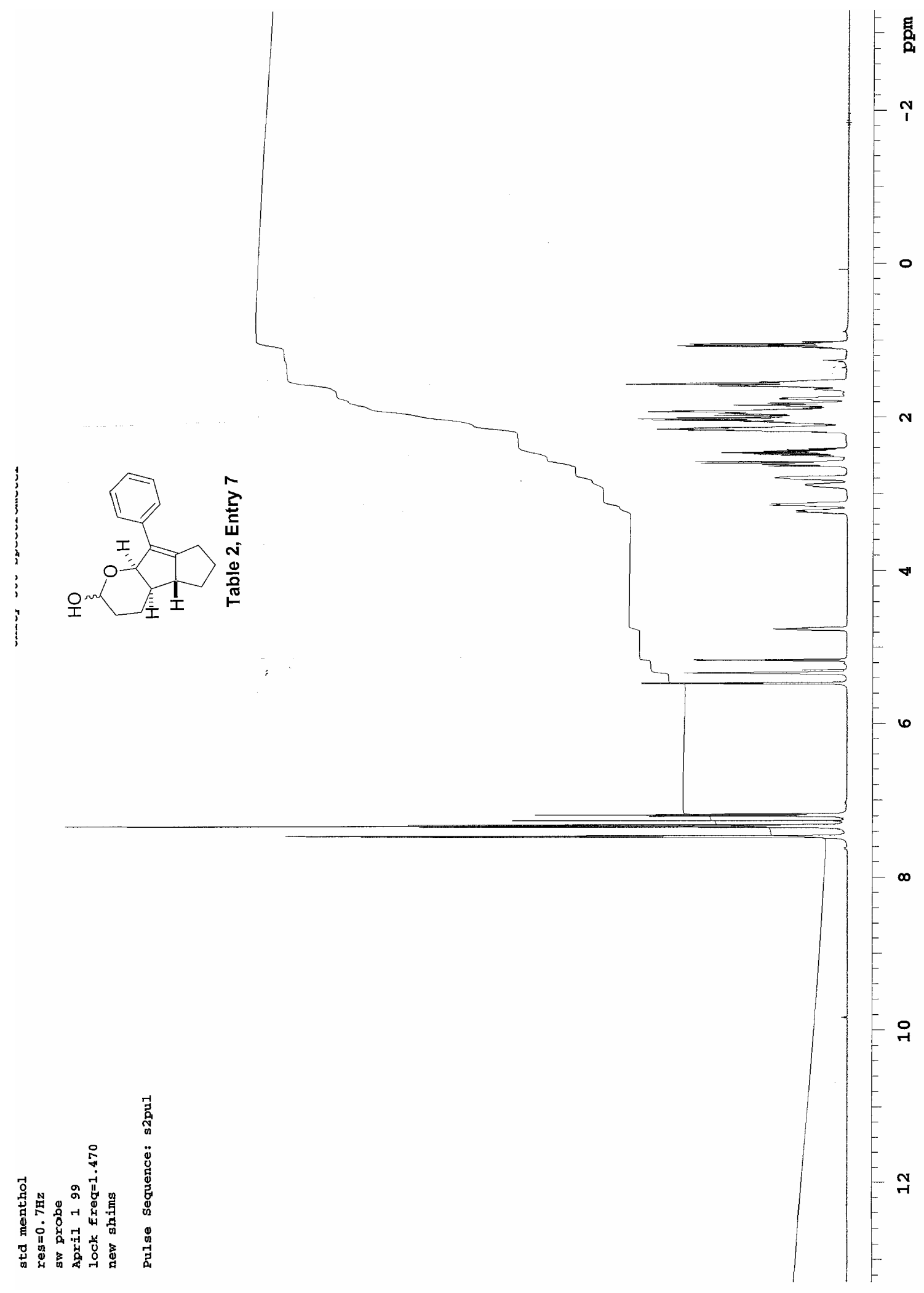




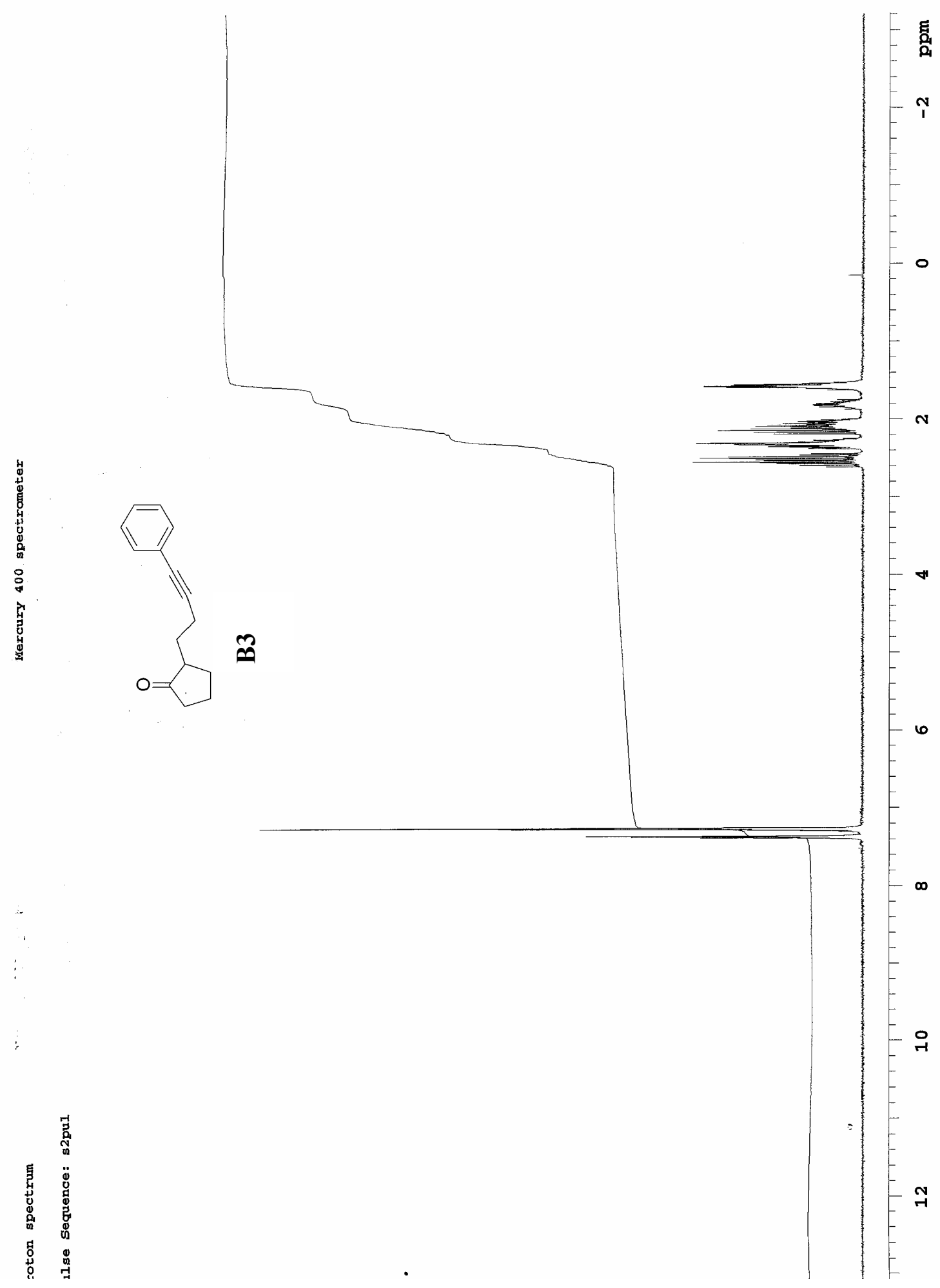




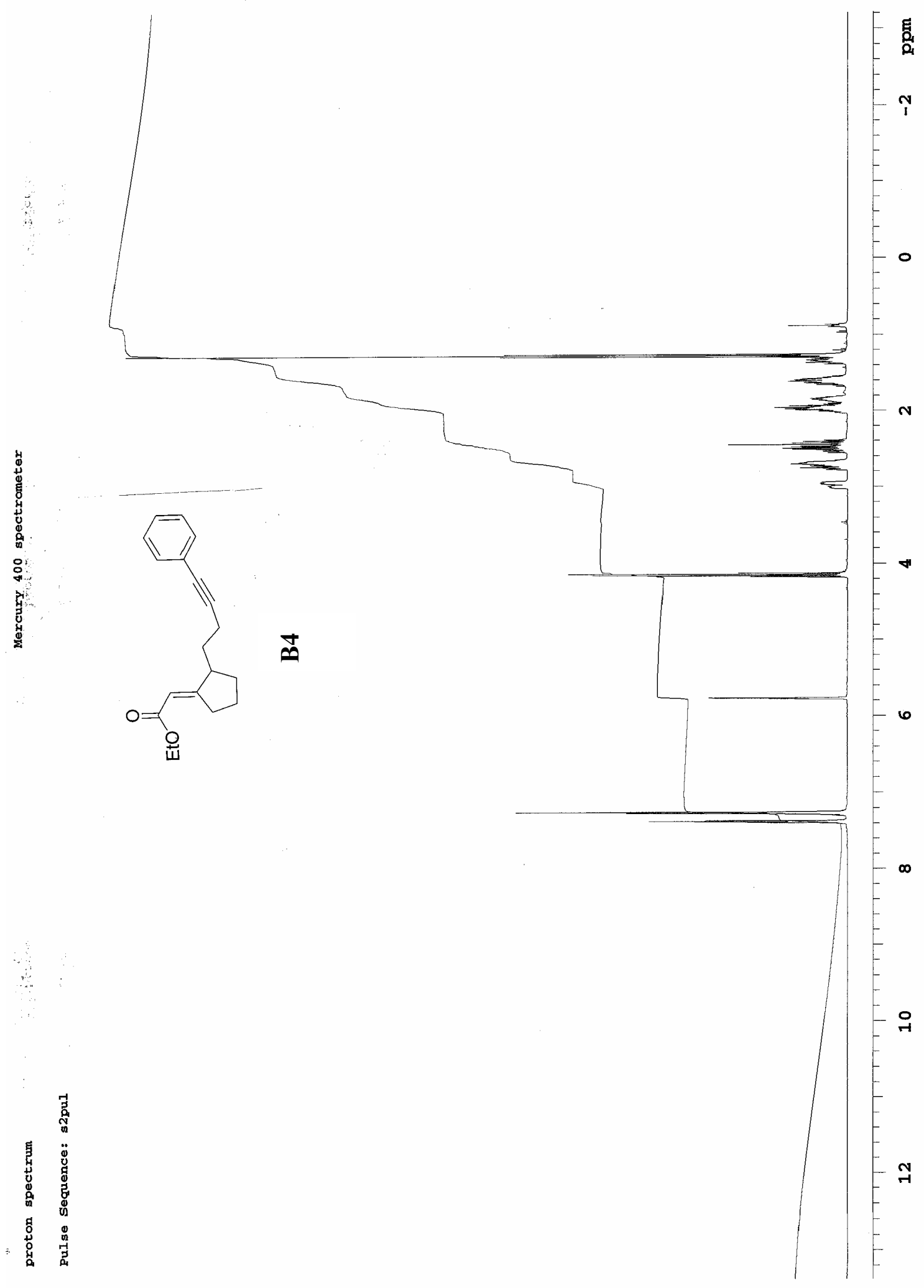




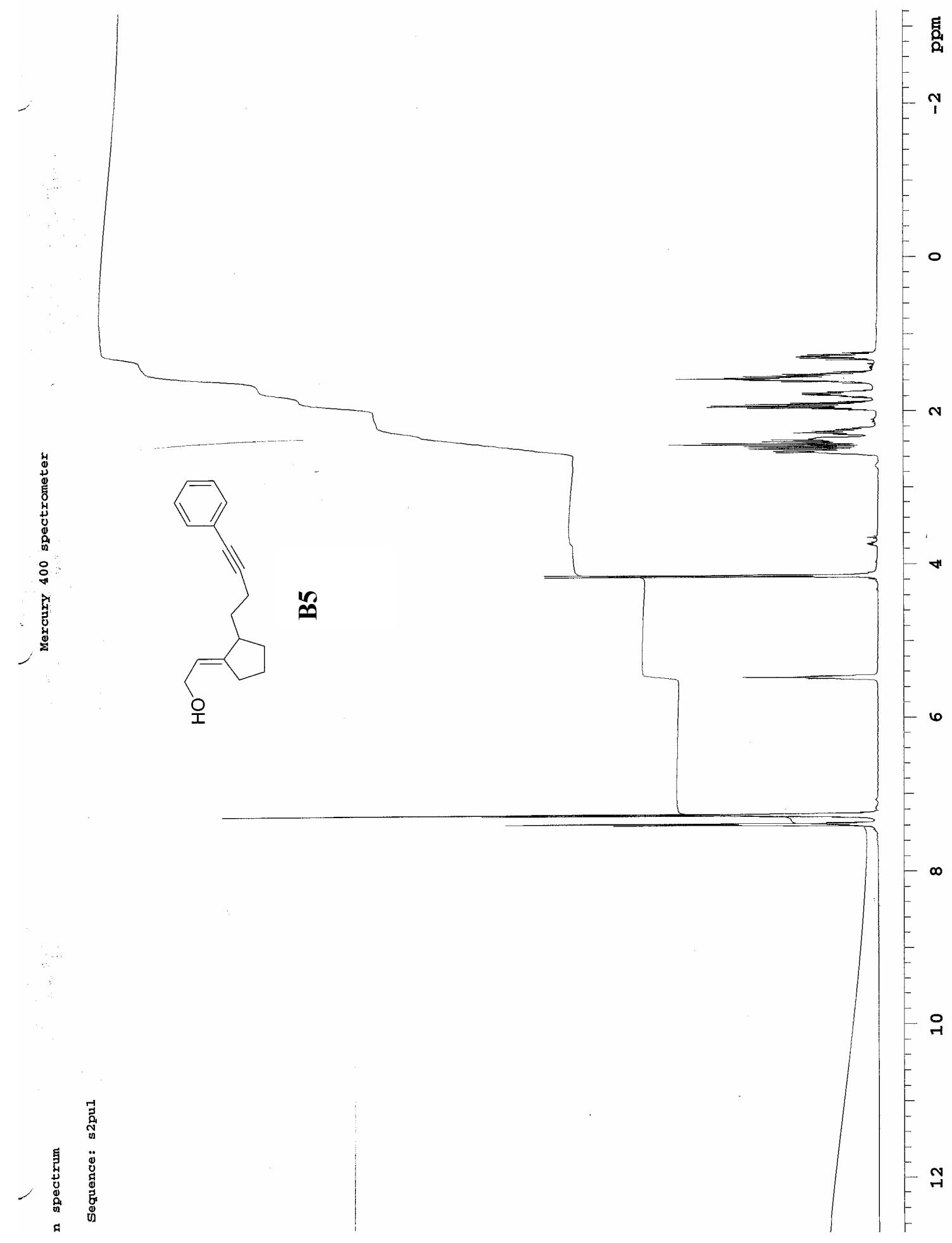




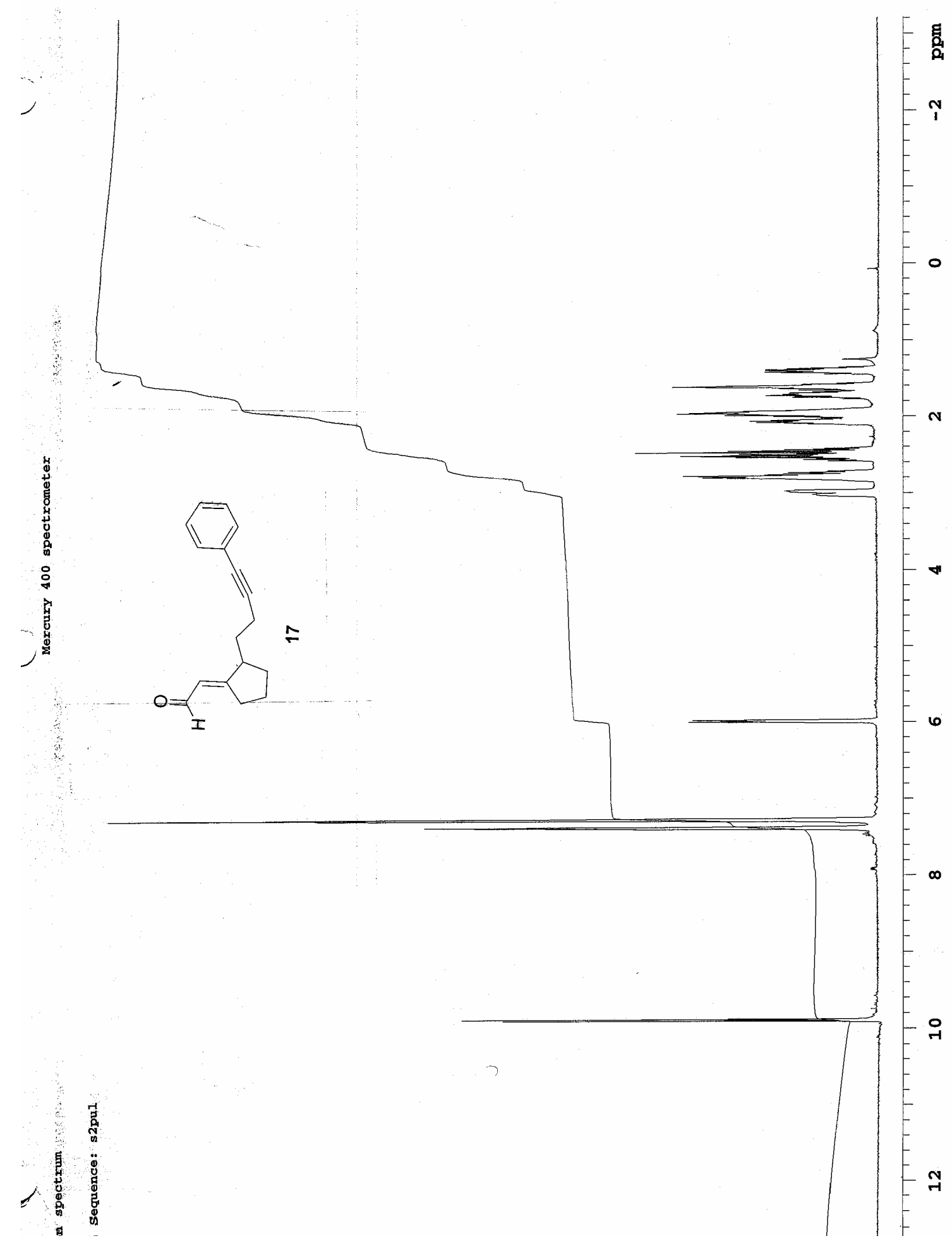




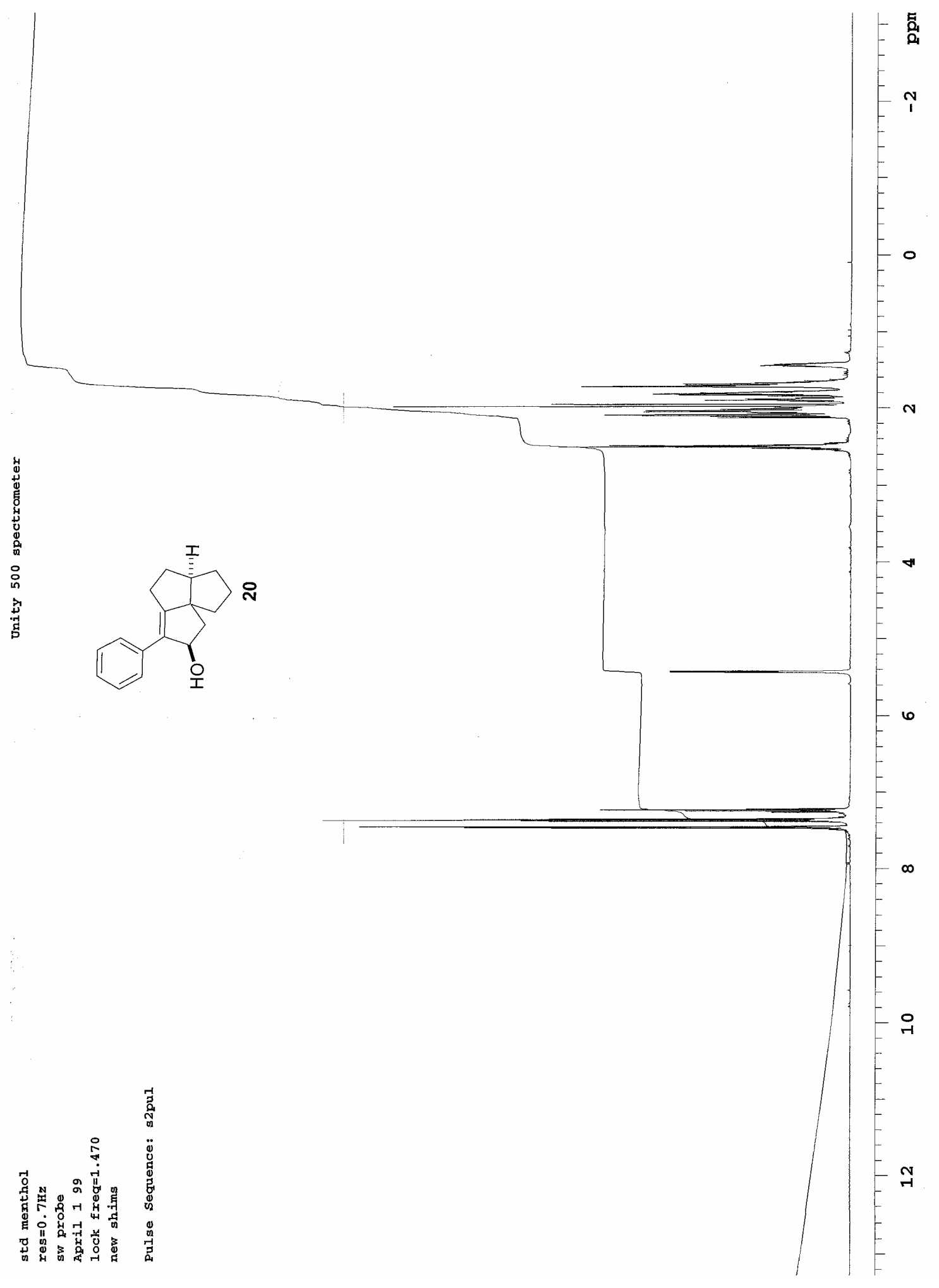




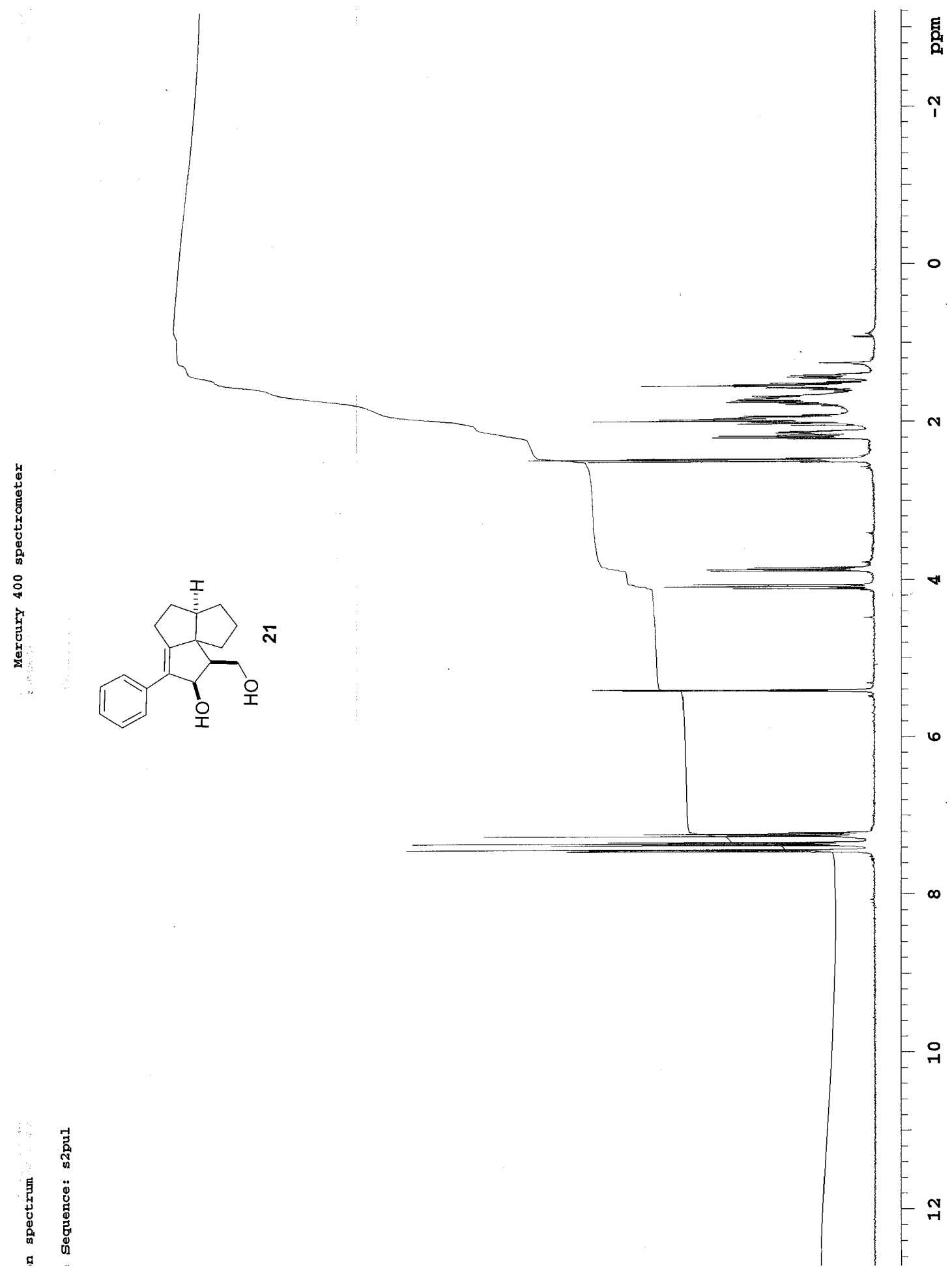




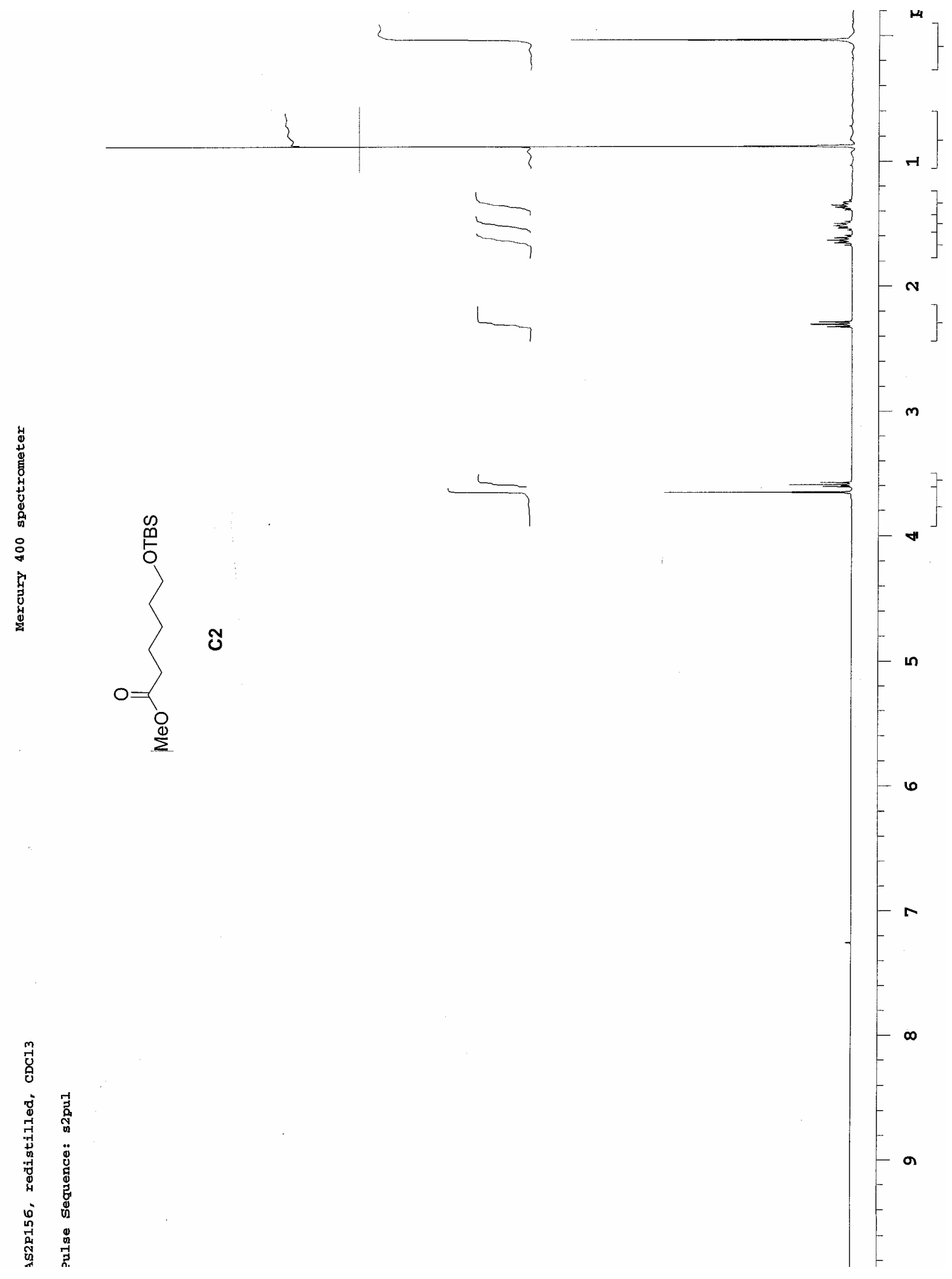




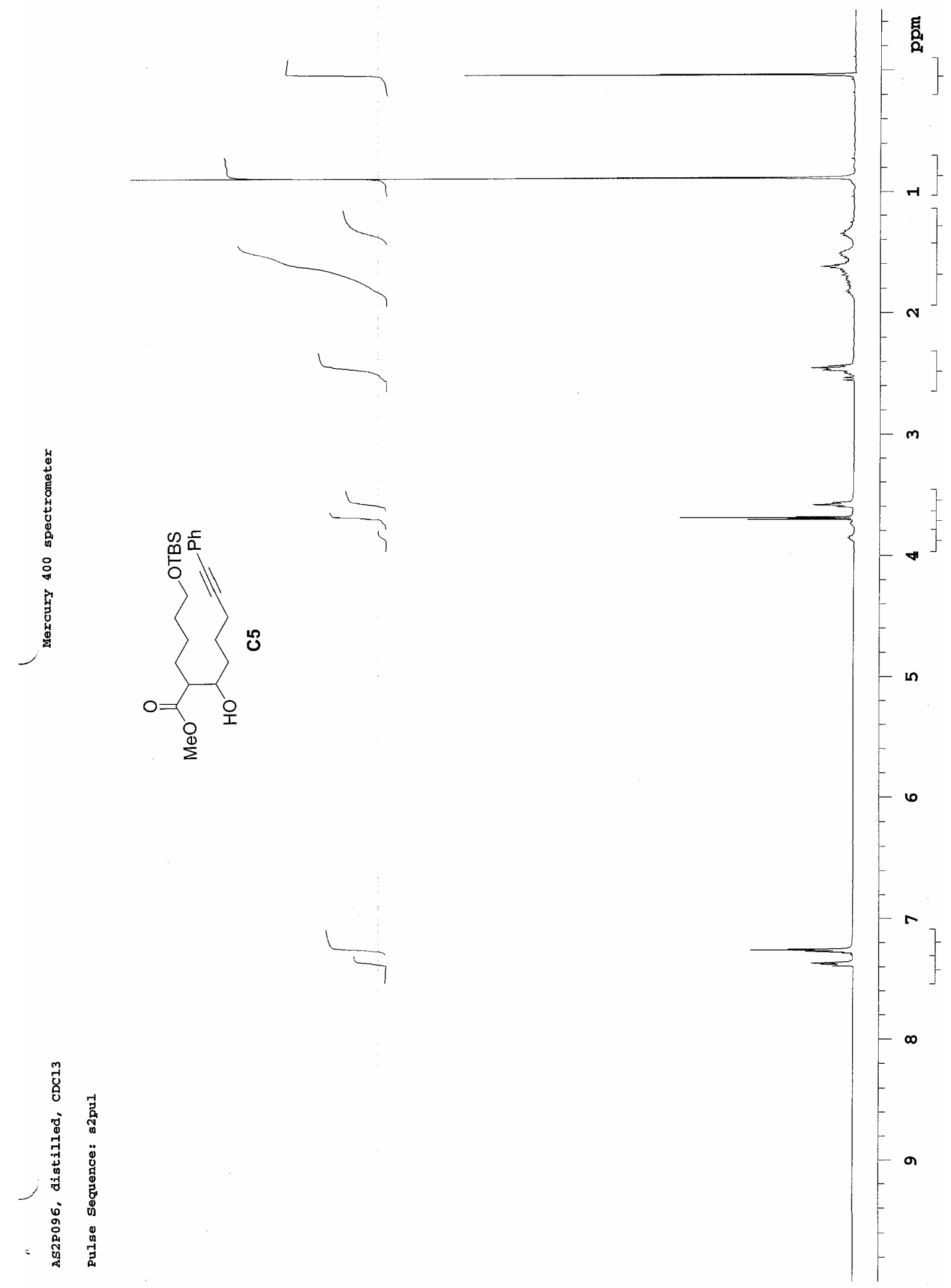




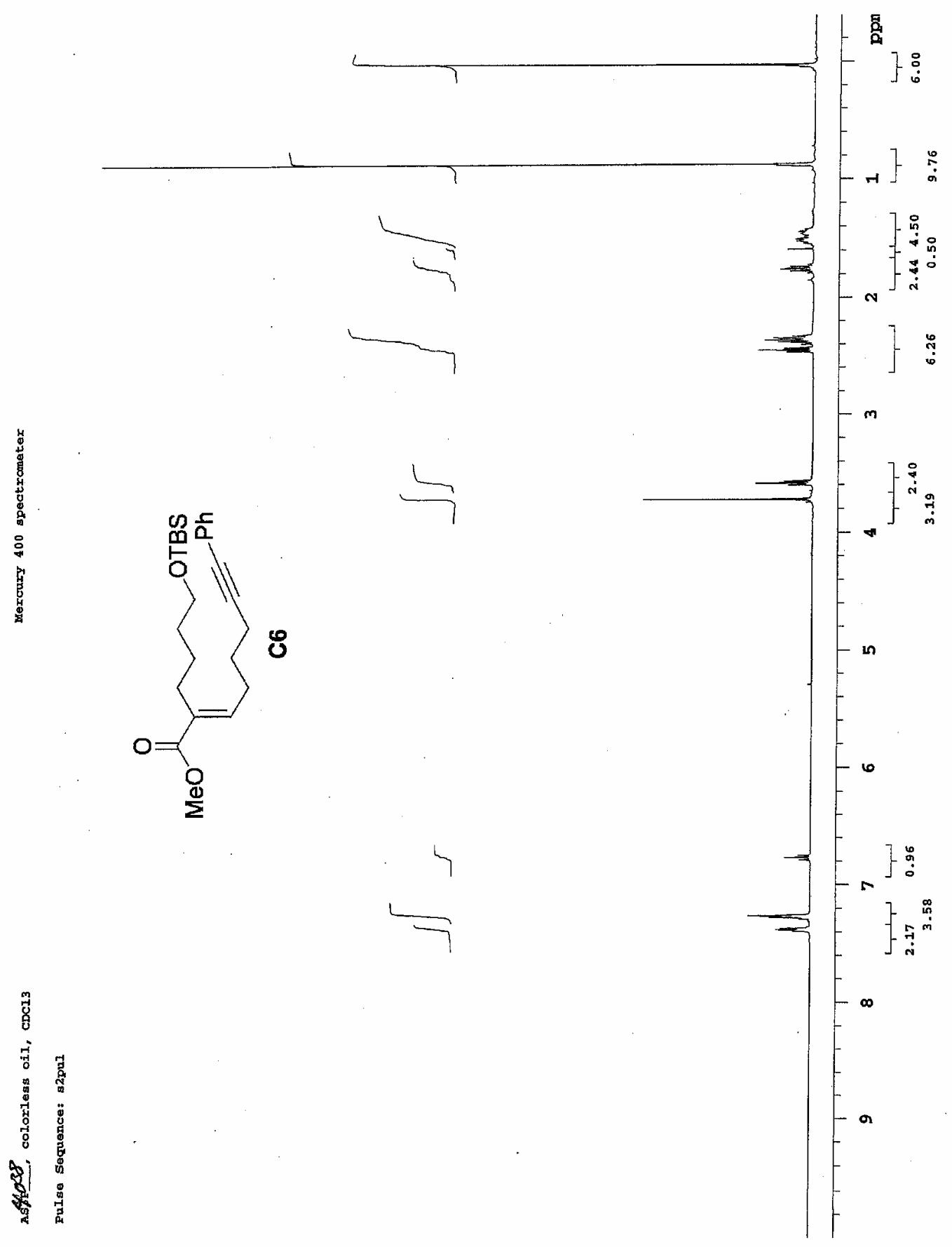




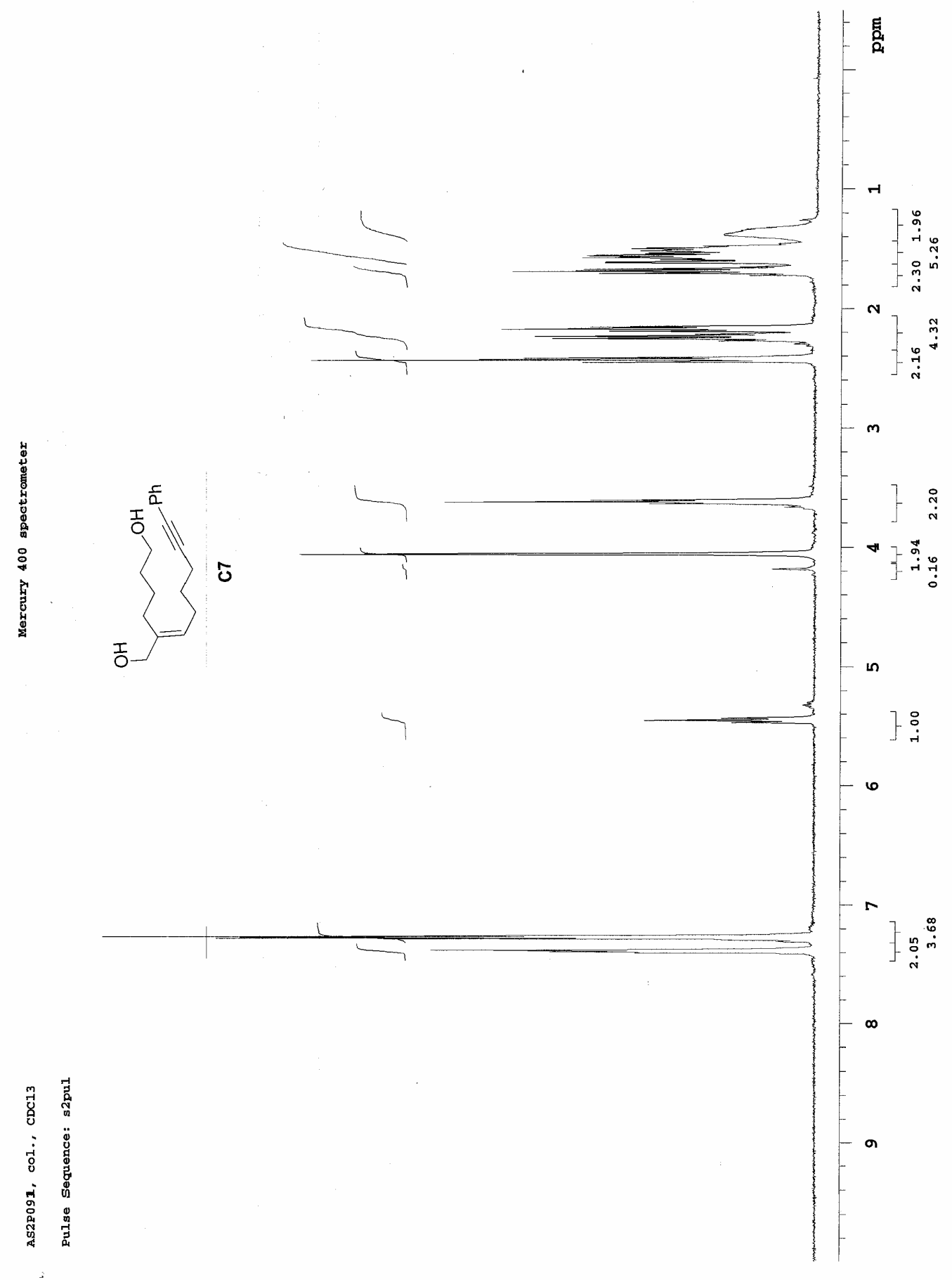




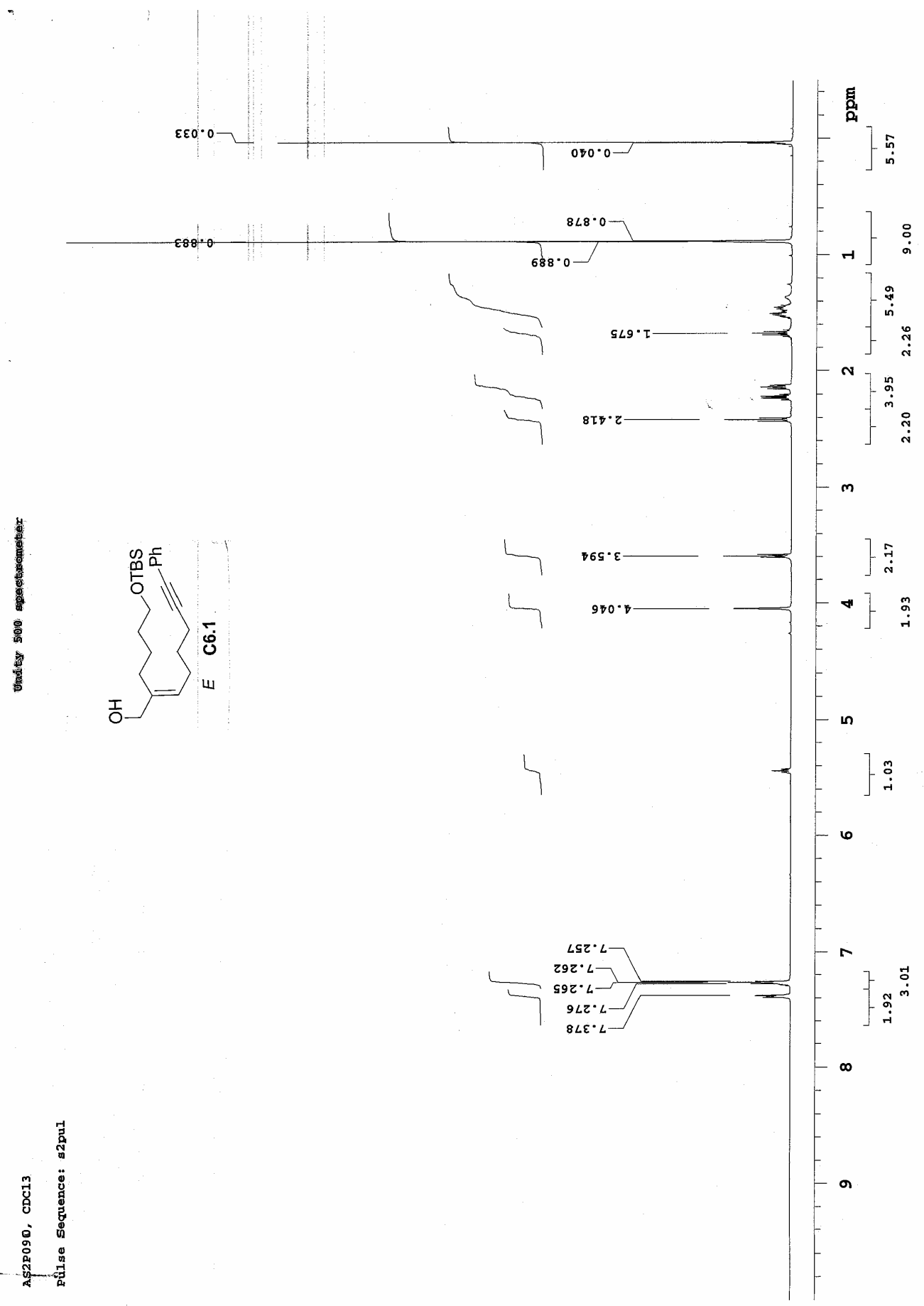




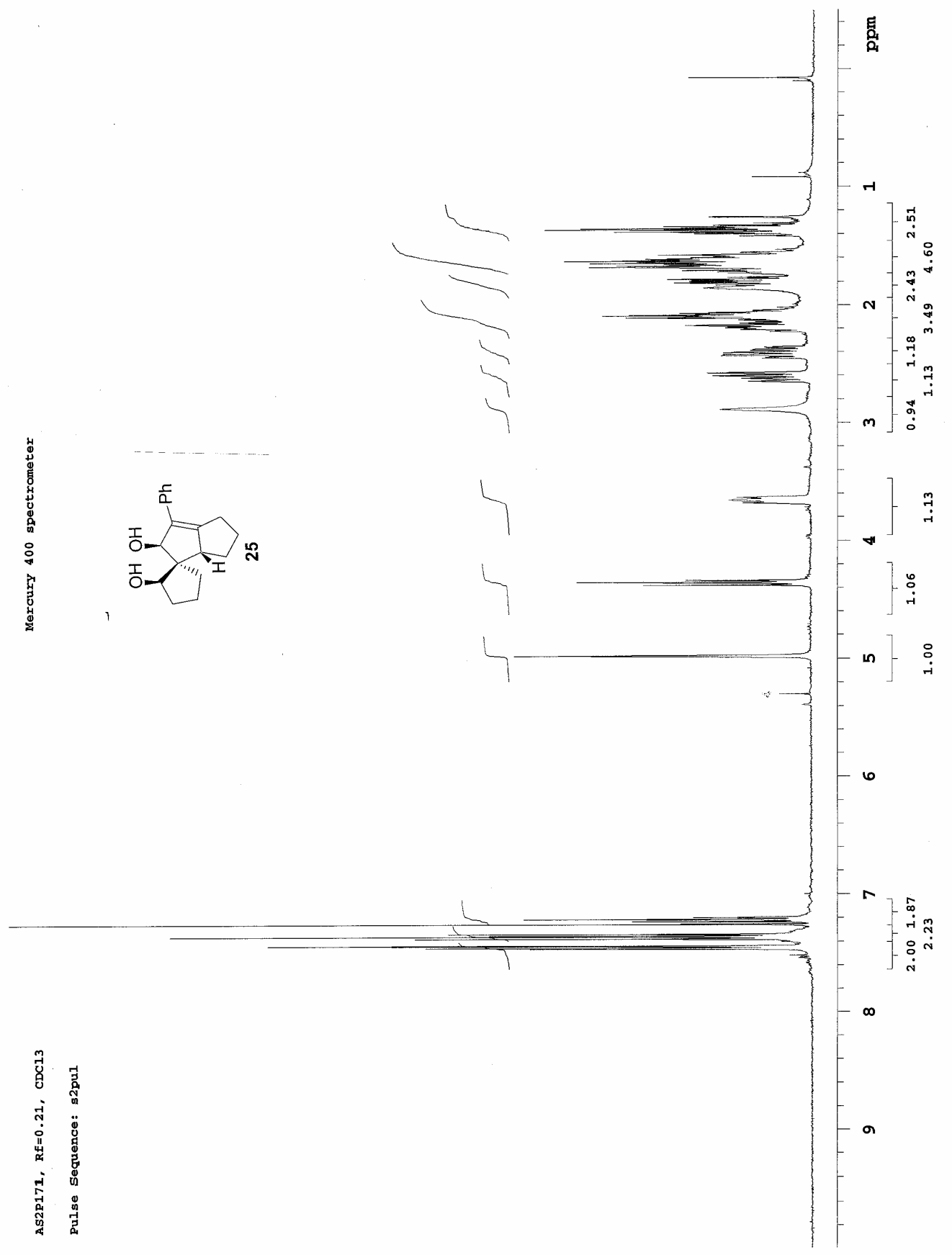




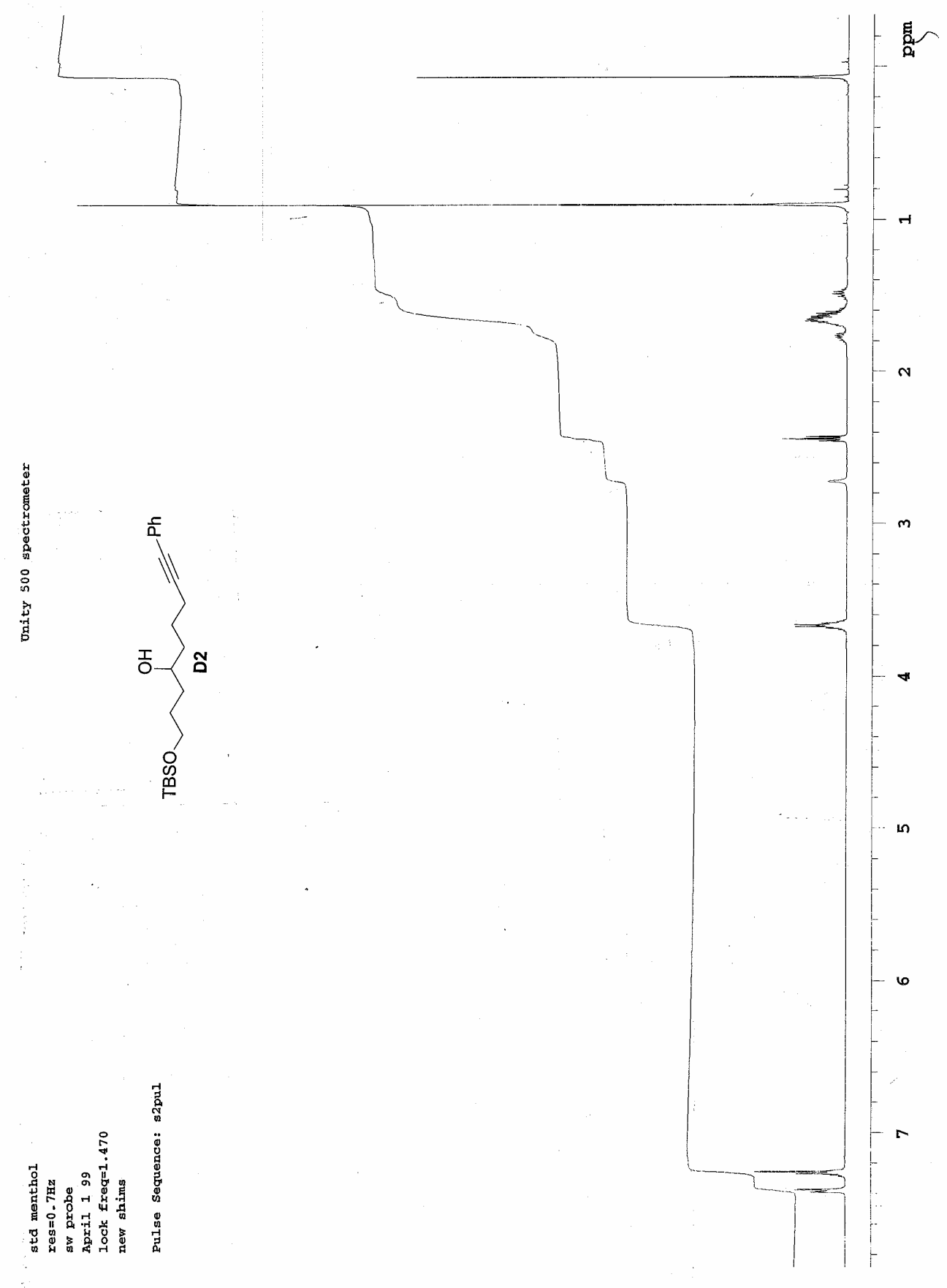




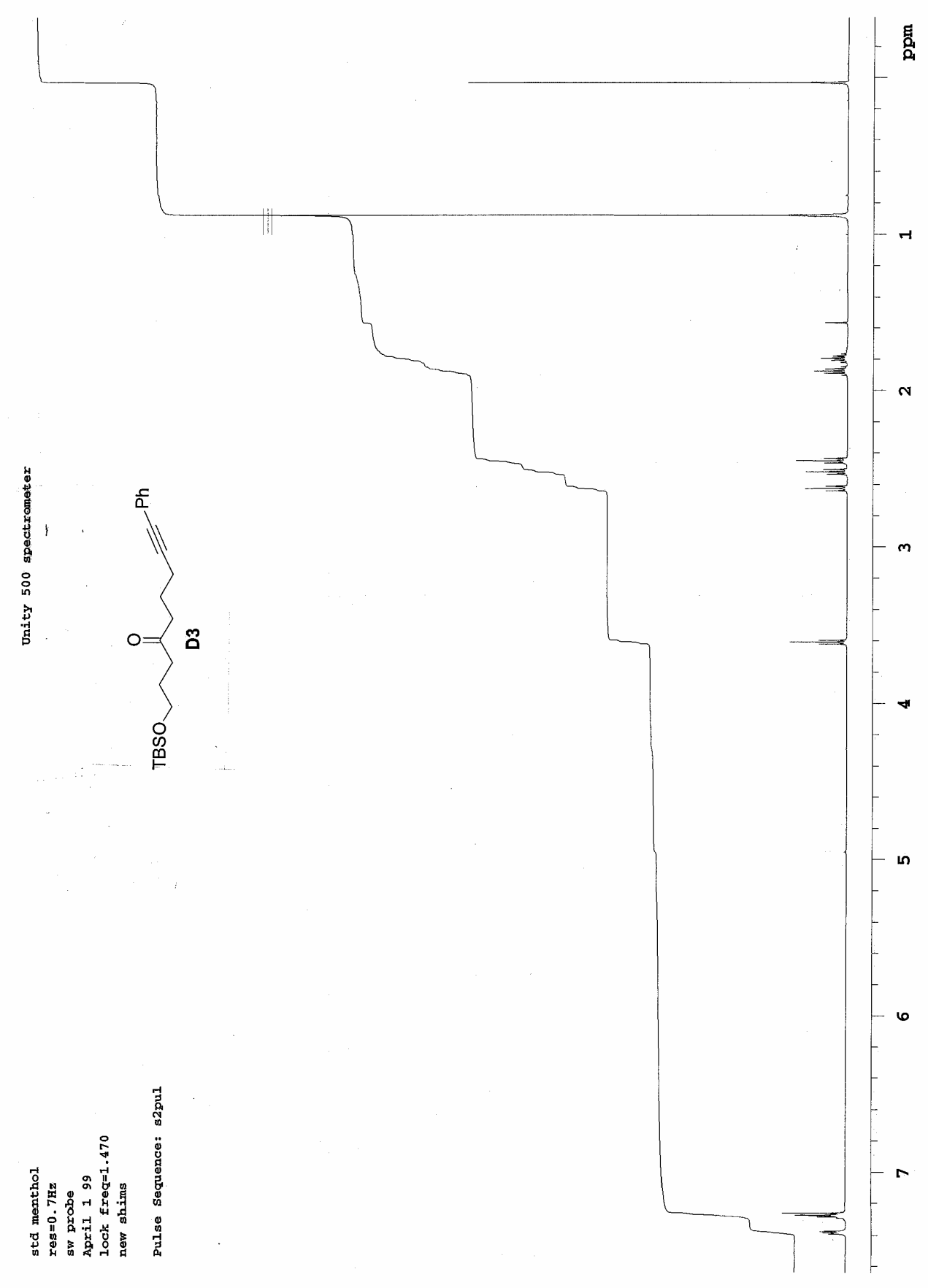




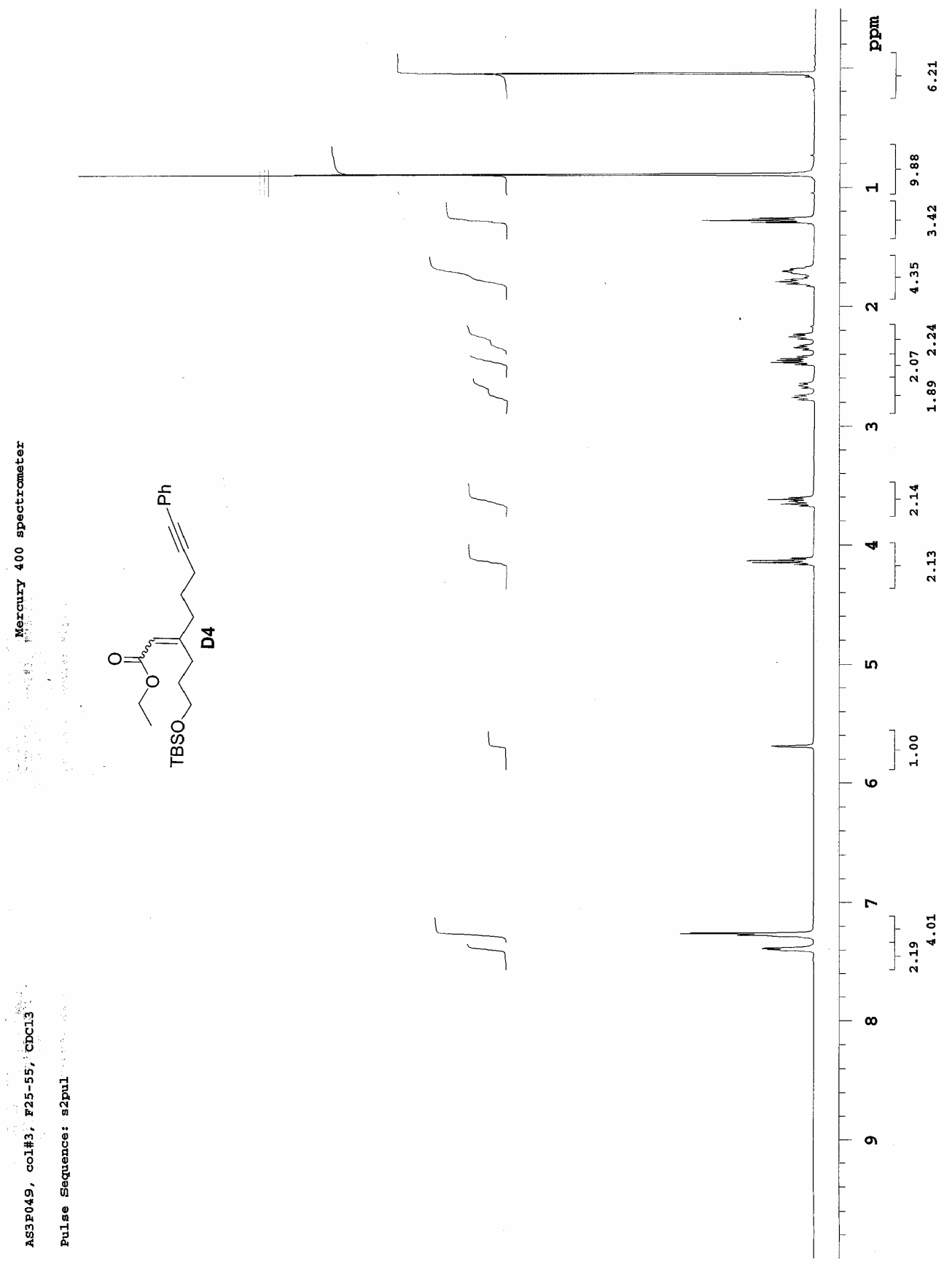




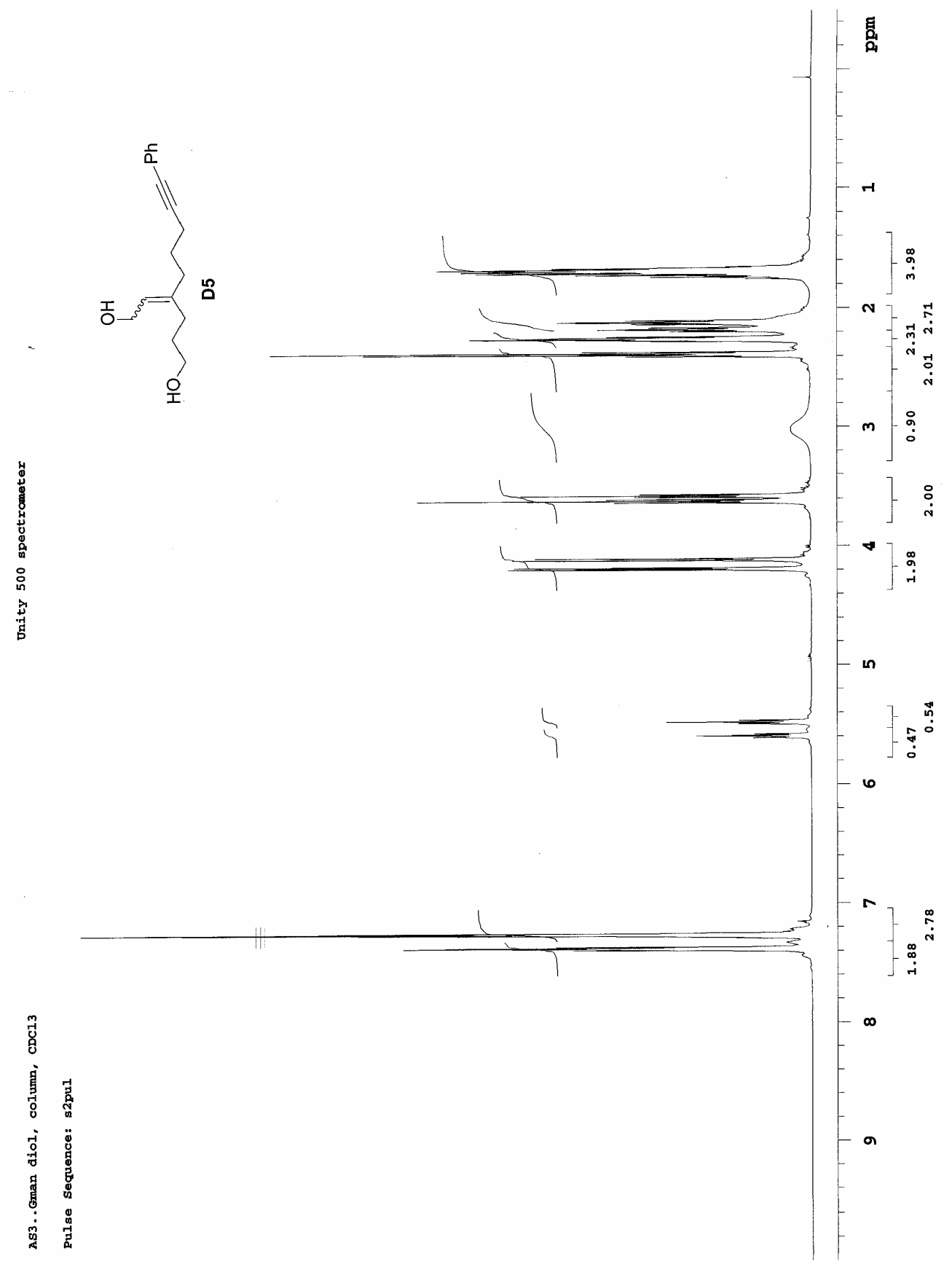




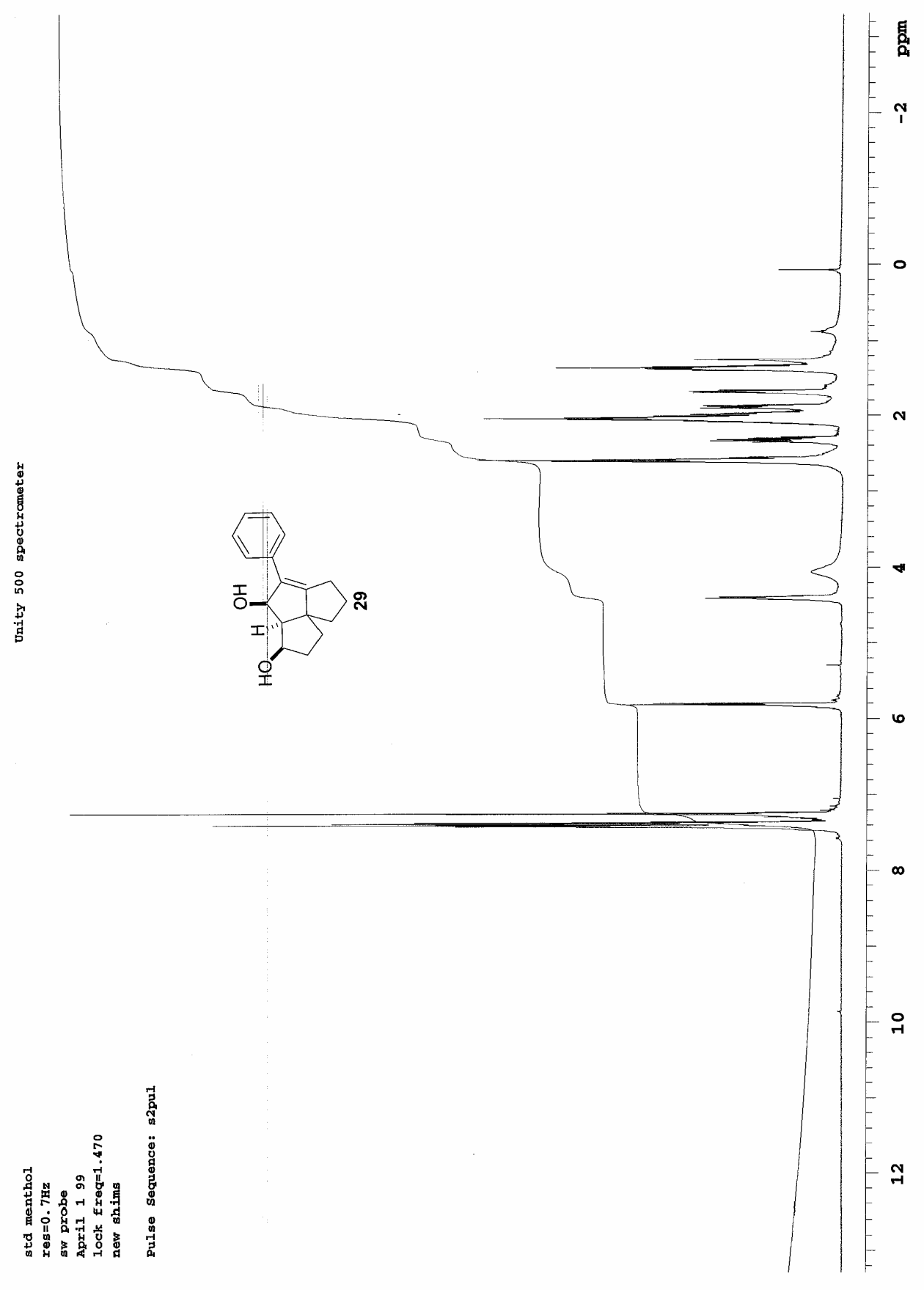

